\title{
THE ALGEBRA OF SECONDARY HOMOTOPY OPERATIONS IN RING SPECTRA
}

\author{
HANS-JOACHIM BAUES AND FERNANDO MURO
}

\begin{abstract}
The primary algebraic model of a ring spectrum $R$ is the ring $\pi_{*} R$ of homotopy groups. We introduce the secondary model $\pi_{*, *} R$ which has the structure of a secondary analogue of a ring. The homology of $\pi_{*, *} R$ is $\pi_{*} R$ and triple Massey products in $\pi_{*, *} R$ coincide with Toda brackets in $\pi_{*} R$. We also describe the secondary model of a commutative ring spectrum $Q$ from which we derive the cup-one square operation in $\pi_{*} Q$. As an application we obtain for each ring spectrum $R$ new derivations of the ring $\pi_{*} R$.
\end{abstract}

\section{Contents}

Introduction

1. Ring spectra and module spectra

2. Pair algebras associated to ring spectra

3. $E_{\infty}$-pair algebras associated to commutative ring spectra

4. Algebras over commutative ring spectra neglecting the Hopf map

5. Square groups and quadratic pair modules

6. Quadratic pair algebras associated to ring spectra

7. Modules over quadratic pair algebras and module spectra

8. Sign group actions on quadratic pair modules

9. $E_{\infty}$-quadratic pair algebras associated to commutative ring spectra

10. Algebras over commutative ring spectra

11. Universal Toda brackets of ring spectra

12. Symmetric spectra

13. Secondary homotopy groups of spaces

14. The pull-back construction

15. Secondary homotopy groups of spectra

16. Proof of Theorems 6.4 7.4 and 9.12

17. Proof of Theorem 3.4

References

\section{INTRODUCTION}

A ring spectrum $R$ is a topological analogue of a ring. The primary algebraic model of $R$ is the ring $\pi_{*} R$ of its homotopy groups. We study in this paper secondary homotopy operations in $\pi_{*} R$ which lead to the secondary model $\pi_{*, *} R$ extending the ring $\pi_{*} R$. The model $\pi_{*, *} R$ has the structure of a secondary algebra which generalizes the notion of algebra in the same sort of way as a 2-group, or crossed module in the sense of Whitehead Whi49, generalizes the classical notion of group by adding a 2-dimensional part. The secondary algebras needed are pair

Date: February 2, 2008.

1991 Mathematics Subject Classification. 55P42, 55Q35, 18 G50.

Key words and phrases. Ring spectrum, homotopy groups, secondary homotopy groups, Toda bracket, Massey product, cup-one product, Shukla cohomology, Mac Lane cohomology, permutative category, quadratic pair module.

The second author was partially supported by the project MTM2004-01865 and the MEC postdoctoral fellowship EX2004-0616. 
algebras (resp. $E_{\infty}$-pair algebras) and quadratic pair algebras (resp. $E_{\infty}$-quadratic pair algebras). They are in a natural way secondary models of ring spectra (resp. commutative ring spectra) and describe new algebraic porperties enriching the commonly used paradigm of ring.

A pair algebra $B$ is a ring $B_{0}$ together with a $B_{0}$-bimodule morphism

$$
\partial: B_{1} \longrightarrow B_{0}
$$

satisfying $\partial(a) b=a \partial(b)$ for $a, b \in B_{1}$. Here $B_{0}, B_{1}$ and $\partial$ are $\mathbb{N}$-graded. In the ungraded case a pair algebra is also termed a crossed bimodule, see $\operatorname{Lod} 98$, E.1.5.1]. Pair algebras or crossed bimodules are known to represent elements in Hochschild and Shukla cohomology, see [Lod98, BP]. Moreover, quadratic pair algebras represent elements in Mac Lane cohomology [BJP06. The cohomology class

$$
\left\langle\pi_{*, *} R\right\rangle \in H M L^{3}\left(\pi_{*} R, \Sigma^{-1} \pi_{*} R\right)
$$

represented by $\pi_{*, *} R$ is the universal Toda bracket ([BD89, Sag06]) which represents the homotopy category of free $R$-modules as a linear extension of categories.

It is well known that homotopy groups $\pi_{*}$ form a lax symmetric monoidal functor carrying the symmetric monoidal category of spectra to the symmetric monoidal category of abelian groups. This, in fact, implies that homotopy groups of a ring spectrum $R$ form a ring. In a similar way we show that there if a lax symmetric monoidal functor $\pi_{*, *}$ on $\Omega$-spectra, given by secondary homotopy groups, which yields the structure of a secondary algebra on $\pi_{*, *} R$. We do not know of any other lax symmetric monoidal functor in the literature leading to such algebraic models of ring spectra extending the ring $\pi_{*} R$.

A functor in the opposite direction is studied by Shipley in Shi06 yielding a ring spectrum $\mathbb{H} D_{*}$ for a differential graded algebra $D_{*}$. In this case $\pi_{*, *} \mathbb{H} D_{*}$ can be described by the secondary algebra associated to $D_{*}$, see Remark 2.8 Moreover, ungraded quadratic pair algebras yield examples of rings and commutative rings in permutative categories, see Remarks 6.9 and 9.14, and Elmendorf and Mandell define in EM05 a functor carrying such objects to ring spectra.

Algebraic Massey products in the secondary algebra $\pi_{*, *} R$ coincide with Toda brackets in $\pi_{*} R$ (Theorems 2.7 and 6.4). Moreover, if $R$ is commutative the algebraic cup-one square in $\pi_{*, *} R$ coincides with the topologycal cup-one square in $\pi_{*} R$ (Theorems 3.4 and 9.12).

The new invariant $\pi_{*, *} R$ is strictly stronger than the collection of secondary operations in $\pi_{*} R$ as we illustrate in Example 3.7 by the 3-local sphere spectrum. This shows examplary computations in the algebraic model $\pi_{*, *} R$.

In case $R_{p}$ is the endomorphism ring spectrum of the Eilenberg-Mac Lane spectrum $H \mathbb{Z} / p$ the ring $\pi_{*} R_{p}=\mathcal{A}$ is the $\bmod p$ Steenrod algebra. In [Bau06] the pair algebra of secondary cohomology operations $\mathcal{B}$ is computed which corresponds to $\pi_{*, *} R_{p}$. It is the purpose of this paper to achieve analogous pair algebras associated to arbitrary ring spectra. As an example the 0 -dimensional part $\pi_{0, *} K \mathbf{W}$ of Waldhausen $K$-theory is computed in MT06.

The sphere spectrum $S$ (which is a connective commutative ring spectrum) yields the $E_{\infty}$-quadratic pair algebra $\pi_{*, *} S$ which enriches the structure of the commutative algebra $\pi_{*} S$ considerably. This $E_{\infty}$-quadratic pair algebra acts on $\pi_{*, *} R$ for any other ring spectrum $R$. The laws of this action (see Definition 10.1) allow the construction of the derivations $\theta(b)$ as follows. Let $b \in \pi_{n} S$ be an element which maps to zero in $\pi_{n} R$ by the unit map $u: S \rightarrow R$ of the ring spectrum $R$. Then a derivation of degree $n$

$$
\theta(b): \pi_{*} R \longrightarrow \Sigma^{-1} \pi_{*} R
$$


is constructued which is well defined up to inner derivation. Moreover $\theta(b)$ is $\pi_{*} S$ linear, so it determines a Hochschild cohomology class

$$
\theta(b) \in H H_{\pi_{*} S}^{1}\left(\pi_{*} R, \Sigma^{-1} \pi_{*} R\right) .
$$

This class vanishes in case $R$ is commutative. The properties of $\theta$ are described in Theorem 1.5,

Special cases of the derivation $\theta(b)$ are known in the literature. For example for the endomorphism spectrum of $H \mathbb{Z} / p$ one has $u_{*}(p \cdot 1)=0$ for $1 \in \pi_{0} S$ and

$$
\theta(p \cdot 1)=\kappa: \mathcal{A} \longrightarrow \Sigma \mathcal{A}
$$

is the Kristensen derivation (Kri63) for $p=2$ which carries $S q^{n}$ to $S q^{n-1}$. For odd primes $\kappa$ is computed in Bau06 by $\kappa\left(P^{n}\right)=0$ and $\kappa(\beta)=1$. Moreover, if $R$ is the endomorphism spectrum of a $\mathbb{Z} / p$-space in the sense of Toda then $\theta(p \cdot 1)$ for $p$ odd coincides with the derivation constructed by Toda in Tod71.

In order to introduce the reader smoothly to the new theory we begin this paper with a section recalling classical secondary homotopy operations and defining the derivations $\theta(b)$ in a topological language. Afterwards we give linear versions of our main theorems for the case of ring spectra neglecting the Hopf map. This will help the reader to understand the more general quadratic versions which are needed to deal with arbitrary spectra.

We are mainly concerned in this paper with connective spectra in order to avoid further technicalities.

\section{Ring SPECTRA AND MOdUlE SPECTRA}

In this paper the framework for stable homotopy theory will be the stable model category of symmetric spectra of compactly generated topological spaces defined in [MMSS01, 9]. The smash product of symmetric spectra $X \wedge Y$ defines a symmetric monoidal structure in this category. The unit of this monoidal structure is the sphere spectrum $S$. Monoids in the category of symmetric spectra are called ring spectra. Ring spectra, modules over a ring spectrum, and algebras over a commutative ring spectrum also form model categories, see [MMSS01, 12]. Fibrant objects in all these categories coincide with the objects which are $\Omega$-spectra. This crucial fact allows the construction of all the new algebraic invariants presented in this paper.

The homotopy groups of a ring spectrum $\pi_{*} R$ carry Toda bracket operations which enrich the ring structure of $\pi_{*} R$. Toda brackets have been considered, for instance, in Tod62 for the sphere spectrum $S$ and in Ale72, under the name of Massey products, for various cobordism spectra.

The homotopy group $\pi_{n} R$ coincides with the group of morphisms $\Sigma^{n} R \rightarrow R$ in the stable homotopy category of right $R$-modules. Therefore Toda brackets in $\pi_{*} R$ are defined following Hel68] by using the triangulated structure in the homotopy category of right $R$-modules, see also Sag06. More precisely, given elements $a, b, c \in \pi_{*} R$ of degree $p, q, r$, respectively, with $a b=0$ and $b c=0$ a generic element in the Toda bracket $\langle a, b, c\rangle \subset \pi_{p+q+r+1} R$ is a morphism $g$ in the homotopy category of right $R$-modules fitting into the commutative diagram

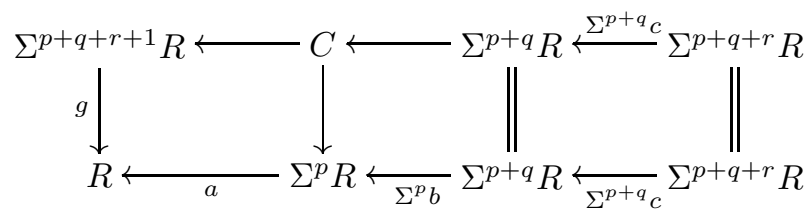

where the upper row is an exact triangle. The existence of such a commutative diagram follows from the axioms of a triangulated category. 
One can also define Toda brackets in $\pi_{*} R$ following BD89 by using tracks in the category of right $R$-modules (i.e. homotopy classes of homotopies between maps). Let us sketch this alternative construction. Since the homotopy groups of any fibrant replacement of $R$ are isomorphic to $\pi_{*} R$ we can suppose without loss of generality that $R$ is a fibrant ring spectrum. In that case the lower row of diagram (1.1) can be realized by a diagram in the category of right $R$-modules

$$
R \longleftarrow \Sigma^{p} R \stackrel{\Sigma^{p} \bar{b}}{\longleftarrow} \Sigma^{p+q} R \stackrel{\Sigma^{p+q} \bar{c}}{\longleftarrow} \Sigma^{p+q+r} R .
$$

The vanishing hypotheses $a b=0$ and $b c=0$ imply the existence of null-homotopies

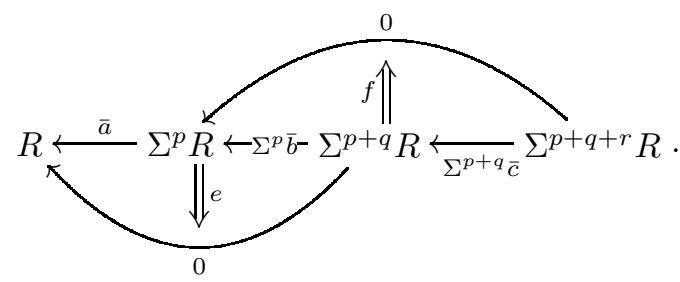

The pasting of this diagram is a self-track of the trivial map $0: \Sigma^{p+q+r} R \rightarrow R$. Such a self-track is the same as a homotopy class

$$
g: \Sigma^{p+q+r+1} R \longrightarrow R,
$$

which is again a generic element of the Toda bracket $\langle a, b, c\rangle$. This is the more convenient approach from the perspective of this paper. More general Toda brackets for a right $R$-module $M,\langle a, b, c\rangle \subset \pi_{p+q+r+1} M$, are defined simply by replacing $R$ by $M$ on the lower left corner of (1.1) or on the left hand side of diagram (1.2), so $a \in \pi_{*} M$ and $b, c \in \pi_{*} R$.

The stable homotopy groups of a commutative ring spectrum $Q$ carry an additional operation, the cup-one square, defined as follows. Let $L Q$ be a fibrant replacement of $Q$ in the category of all ring spectra. The $\operatorname{ring}$ spectrum $L Q$ is no longer commutative, but it remains commutative up to a coherent track $\alpha_{1}$ satisfying the idempotence and the hexagon axioms for symmetric monoidal categories, compare Lemma 16.2. Given $a \in \pi_{2 n} Q, n \geq 0$, we take a representative $\bar{a}: S^{2 n} \rightarrow L Q$ where the spectrum $S^{m}$ is the $m$-fold suspension of the sphere spectrum $S, S^{m}=\Sigma^{m} S$. The symmetry isomorphism for the smash square of an even-dimensional sphere $\tau_{\wedge}: S^{2 n} \wedge S^{2 n} \cong S^{2 n} \wedge S^{2 n}$ is homotopic to the identity. We can choose a track $\hat{\tau}: \tau_{\wedge} \Rightarrow 1_{S^{2 n} \wedge S^{2 n}}$, there are two such choices. Consider the following diagram where $\mu$ is the product in $L Q$.

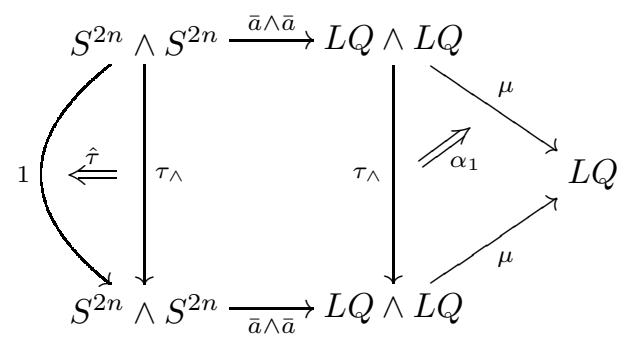

The pasting of this diagram is a self-track of $\mu(\bar{a} \wedge \bar{a})$. The classical Barcus-BarrattRutter isomorphism allows us to indetify this self-track with a homotopy class

$$
S q_{1}(a): S^{4 n+1}=\Sigma\left(S^{2 n} \wedge S^{2 n}\right) \longrightarrow Q
$$

measuring the difference between the pasting of (1.3) and the identity self-track on $\mu(\bar{a} \wedge \bar{a})$. This element $S q_{1}(a) \in \pi_{4 n+1} Q$ is the cup-one square of $a$. One can check 
that $S q_{1}(a)$ does not depend on the representative $\bar{a}$. However in general it does depend on the choice of $\hat{\tau}$.

Assume now that $R$ is a $Q$-algebra. The main example is $Q=S$ since all ring spectra are algebras over the sphere spectrum. Let $u: Q \rightarrow R$ be the unit, let $\tau_{\wedge_{Q}}$ be the symmetry isomorphism for the smash product of $Q$-modules $\wedge_{Q}$, and let $\bar{L}$ be a fibrant replacement functor in the category of $Q$-algebras. The diagram

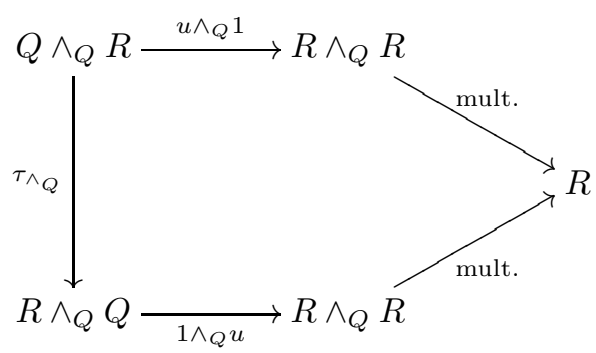

commutes but

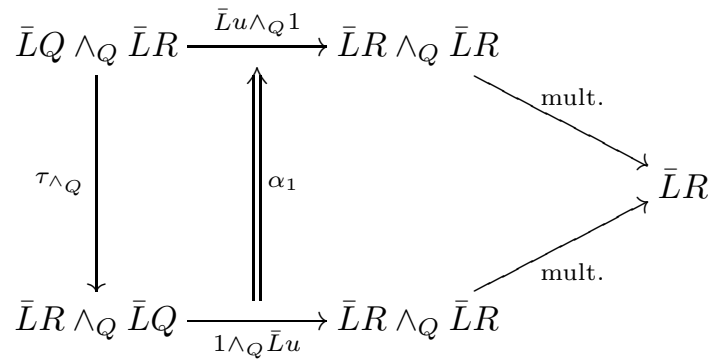

is only commutative up to a certain track $\alpha_{1}$, compare Remark 10.7 .

If $b \in \pi_{n} Q$ is in the kernel of the homomorphism $\pi_{*} Q \rightarrow \pi_{*} R$ induced by the unit map $u: Q \rightarrow R$ we can choose a representative $x: S^{n} \rightarrow \bar{L} Q$ and a track $y:(\bar{L} u) x \Rightarrow 0$. Given $a \in \pi_{m} R$ represented by $\bar{a}: S^{m} \rightarrow \bar{L} R$ the pasting of the diagram

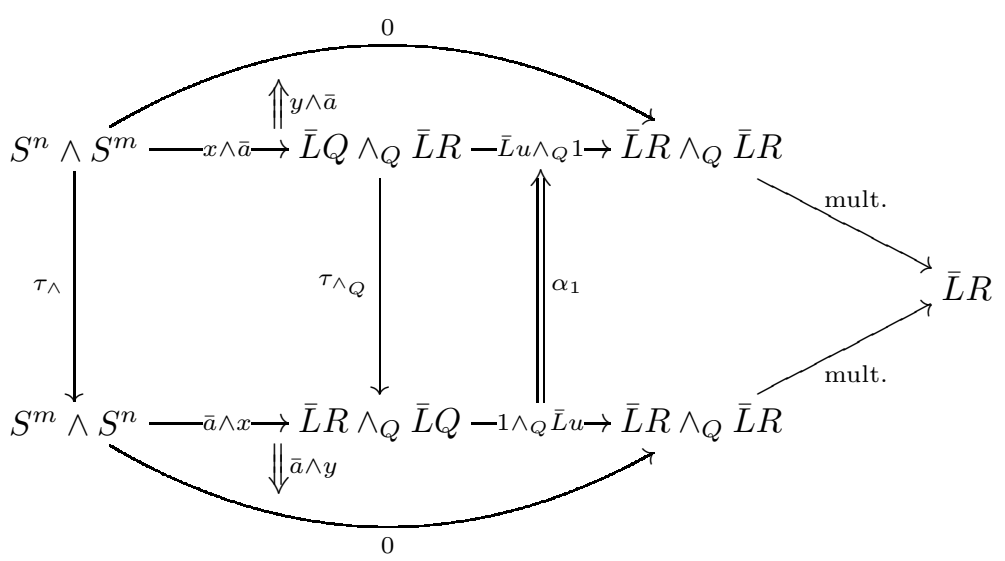

is a selft track of 0: $S^{n+m}=S^{n} \wedge S^{m} \rightarrow \bar{L} R$, or equivalently an element

$$
\theta_{(x, y)}(a) \in \pi_{n+m+1} R \text {. }
$$

One can easily check that this element does not depend on the choice of $\bar{a}$. In Theorem 10.6 we identify $\theta_{(x, y)}(a)$ in a purely algebraic way for connective spectra. This allows to deduce that

$$
\theta_{(x, y)}: \pi_{*} R \longrightarrow \Sigma^{-1} \pi_{*} R
$$


is a degree $n$ derivation of the graded ring $\pi_{*} R$ with coefficients in the desuspended bimodule $\Sigma^{-1} \pi_{*} R$. Moreover, Proposition 10.5 implies the following theorem.

Theorem 1.5. Let $Q$ be a connective commutative ring spectrum, let $R$ be a connective $Q$-algebra, and let $I_{Q}(R)$ be the kernel of the ring homomorphism $\pi_{*} u: \pi_{*} Q \rightarrow$ $\pi_{*} R$. Then there is a $\pi_{*} Q$-module homomorphism

$$
\theta: I_{Q}(R) / I_{Q}(R)^{2} \longrightarrow H H_{\pi_{*} Q}^{1}\left(\pi_{*} R, \Sigma^{-1} \pi_{*} R\right), \quad b+I_{Q}(R)^{2} \mapsto\left\{\theta_{(x, y)}\right\} .
$$

This homomorphism is natural in $R$ and in $Q$ in the obvious way. This implies that $\theta$ vanishes when $R$ is commutative.

All ring spectra are $S$-algebras so the theorem applies to all connective ring spectra for $Q=S$ the sphere spectrum. The example of $\theta(p \cdot 1)$ of Toda shows that $\theta$ is non-trivial, see Tod71.

For a brief remainder of 1-dimensional Hochschild cohomology see the paragraph preceding Definition 4.10 ,

In Section 12 we review more technical details about spectra which are needed for the proofs of the main results in this paper.

\section{Pair algebras Associated to Ring SPECTRA}

Let pm be the category of chain complexes of abelian groups concentrated in degree 0 and 1 . Such chain complexes are termed pair modules, see Bau06, and are denoted by

$$
M=\left(\partial: M_{1} \rightarrow M_{0}\right) .
$$

The tensor product of two pair modules $M \bar{\otimes} N$ is obtained from the tensor product of chain complexes $M \otimes N$ by quotienting out the subcomplex generated by elements in dimension 2, compare BMR04. Hence the category pm is symmetric monoidal. The unit object is $\mathbb{Z}=(0 \rightarrow \mathbb{Z})$. Quasi-isomorphisms in pm are morphisms inducing isomorphisms on the "homology" functors,

$$
h_{0}, h_{1}: \mathbf{p m} \longrightarrow \mathbf{A b},
$$

defined by

$$
\begin{aligned}
h_{0} M & =\operatorname{Coker}\left(\partial: M_{1} \rightarrow M_{0}\right), \\
h_{1} M & =\operatorname{Ker}\left(\partial: M_{1} \rightarrow M_{0}\right) .
\end{aligned}
$$

These homology functors come equipped with a natural isomorphism

$$
h_{0}(M) \otimes h_{0}(N) \cong h_{0}(M \bar{\otimes} N),
$$

and with natural pairings

$$
\begin{aligned}
& h_{0}(M) \otimes h_{1}(N) \longrightarrow h_{1}(M \bar{\otimes} N), \\
& h_{1}(M) \otimes h_{0}(N) \longrightarrow h_{1}(M \bar{\otimes} N) .
\end{aligned}
$$

The isomorphism (2.2) and the pairings (2.3) satisfy the obvious associativity and commutativity properties, i.e. they define a lax symmetric monoidal structure on the functor $\left(h_{0}, h_{1}, 0, \ldots\right)$ from $\mathbf{p m}$ to the category of $\mathbb{N}$-graded abelian groups where $\mathbb{N}=\{0,1,2, \ldots\}$.

We mostly deal with $\mathbb{N}$-graded objects. An ungraded object is an $\mathbb{N}$-graded object concentrated in degree 0 .

The category $\mathbf{p m}^{\mathbb{N}}$ of $\mathbb{N}$-graded pair modules $M=\left(M_{n, *}, n \in \mathbb{N}\right)$ is also symmetric monoidal with the tensor product

$$
(M \bar{\otimes} N)_{n, *}=\bigoplus_{i+j=n} M_{i, *} \bar{\otimes} N_{j, *}
$$


The symmetry isomorphism $\tau_{\bar{\otimes}}: M \bar{\otimes} N \cong N \bar{\otimes} M$ in $\mathbf{p m}^{\mathbb{N}}$ is defined by $a_{p, q} \otimes b_{r, s} \mapsto$ $(-1)^{p r} b_{r, s} \otimes a_{p, q}$. Here the first subscript of $a_{p, q} \in M_{p, q}$ will always denote the $\mathbb{N}-$ grading and the second one the $\{0,1\}$-grading of the corresponding pair module $M_{p, *}$. We point out that $a_{p, 1} \otimes b_{r, 1}$ represents 0 in $M \bar{\otimes} N$.

A pair algebra is a monoid in $\mathbf{p m}^{\mathbb{N}}$. It is given by an $\mathbb{N}$-graded ring $B_{*, 0}$, an $\mathbb{N}$-graded $B_{*, 0}$-bimodule $B_{*, 1}$, and a $B_{*, 0}$-bimodule homomorphism $\partial: B_{*, 1} \rightarrow B_{*, 0}$ such that

$$
a \cdot \partial(b)=\partial(a) \cdot b, \quad a, b \in B_{*, 1} .
$$

If $B$ is a pair algebra then by using (2.2) and (2.3) $h_{0} B$ is an $\mathbb{N}$-graded ring and $h_{1} B$ is an $h_{0} B$-bimodule in a natural way.

Let $A$ be a ring. The most basic examples of pair algebras are the inclusion of a two-sided ideal $I \subset A$ and the zero morphism $0: M \rightarrow A$ where $M$ is an $A$-bimodule. An ungraded pair algebra is also termed a "crossed bimodule", see Lod98, E.1.5.1].

Remark 2.5. Given any ring $A$ and any $A$-bimodule $M$ consider the category consisting of pair algebras $B$ together with a ring isomorphism $h_{0} B \cong A$ and a bimodule isomorphism $h_{1} B \cong M$. Morphisms are pair algebra morphisms over $A$ and under $M$. Then the set of connected components of this category is in natural bijection with 3-dimensional Shukla cohomology

$$
S H^{3}(A, M),
$$

see $\mathrm{BP}$. Shukla cohomology is derived Hochschild cohomology. If the inclusions $h_{1} B \subset B_{1}$ and $\partial\left(B_{1}\right) \subset B_{0}$ split additively then the Shukla cohomology class associated to a pair algebra $B$ is in the image of the natural homomorphism from Hochschild cohomology

$$
H H^{3}(A, M) \longrightarrow S H^{3}(A, M),
$$

see [BP] and [Lod98, Exercise E.1.5.1].

There is a notion of Massey product for pair algebras which is defined as follows.

Definition 2.6. Let $B$ be a pair algebra. Given elements $a, b, c \in h_{0} B$ of degree $p, q, r \in \mathbb{N}$ with $a b=0$ and $b c=0$ the Massey product is the subset

$$
\langle a, b, c\rangle \subset h_{1} B_{p+q+r}
$$

which is a coset of the subgroup

$$
\left(h_{1} B_{p+q}\right) c+a\left(h_{1} B_{q+r}\right)
$$

defined as follows. Given $\bar{a} \in B_{p, 0}, \bar{b} \in B_{q, 0}, \bar{c} \in B_{r, 0}$ representing $a, b, c$, there exist $e \in B_{p+q, 1}, f \in B_{q+r, 1}$ such that $\partial(e)=\bar{a} \cdot \bar{b}, \partial(f)=\bar{b} \cdot \bar{c}$. Then one can easily check that

$$
-e \cdot \bar{c}+\bar{a} \cdot f \in h_{1} B_{p+q+r} \subset B_{p+q+r, 1} .
$$

The coset $\langle a, b, c\rangle \subset h_{1} B_{p+q+r}$ coincides with the set of elements obtained in this way for all different choices of $\bar{a}, \bar{b}, \bar{c}, e$ and $f$.

We say that a ring spectrum $R$ neglects the Hopf map if the stable Hopf map $\eta \in \pi_{1} S$ is in the kernel of the ring homomorphism $\pi_{*} S \rightarrow \pi_{*} R$ induced on stable homotopy groups by the unit $S \rightarrow R$.

Recall that the desuspension $\Sigma^{-1} A$ of an $\mathbb{N}$-graded ring $A$ is $\left(\Sigma^{-1} A\right)_{n}=A_{n+1}$, $n \geq 0$, with the $\mathbb{N}$-graded $A$-bimodule structure defined by the formula

$$
a \cdot\left(\Sigma^{-1} b\right) \cdot c=(-1)^{|a|} \Sigma^{-1}(a \cdot b \cdot c) .
$$

Here $|a|$ is the degree of $a \in A$ and for any $x \in A_{n+1}, n \geq 0$, we denote by $\Sigma^{-1} x \in\left(\Sigma^{-1} A\right)_{n}$ the corresponding element in the desuspension. One of our main results is the following. 
Theorem 2.7. There is a functor

$$
\pi_{*, *}^{a d d}:\left(\begin{array}{c}
\text { connective ring } \\
\text { spectra neglecting } \eta
\end{array}\right) \longrightarrow \text { (pair algebras) }
$$

together with natural isomorphisms

$$
\begin{aligned}
& h_{0} \pi_{*, *}^{a d d} R \cong \pi_{*} R, \text { of rings, } \\
& h_{1} \pi_{*, *}^{a d d} R \cong \Sigma^{-1} \pi_{*} R, \text { of bimodules },
\end{aligned}
$$

such that the Massey products in $\pi_{*, *}^{a d d} R$ coincide with the Toda brackets in $\pi_{*} R$.

Proof. The functor $\pi_{*, *}^{a d d}$ is defined by the formula $\pi_{*, *}^{a d d} R=\left(\pi_{*, *} R\right)^{\text {add }}$ where $\pi_{*, *}$ is the functor in Theorem 6.4 and $(-)^{a d d}$ is the additivization functor in (5.6). Now the theorem follows from Theorem 6.4 and Proposition 5.7.

Example 2.8. Common examples of connective ring spectra neglecting the Hopf map are the spectra associated to multiplicative cohomology theories concentrated in even non-negative dimensions, such as connective complex $K$-theory $k u$, complex cobordism $M U$, Brown-Peterson theory $B P \ldots$ If $R$ denotes any of these ring spectra then the pair algebra $\pi_{*, *}^{a d d} R$ is quasi-isomorphic to

$$
0: \Sigma^{-1} \pi_{*} R \longrightarrow \pi_{*} R \text {. }
$$

In fact if $B$ is any pair algebra such that $h_{0} B$ is concentrated in even degrees and $h_{1} B$ is concentrated in odd degrees then we have a diagram of quasi-isomorphisms

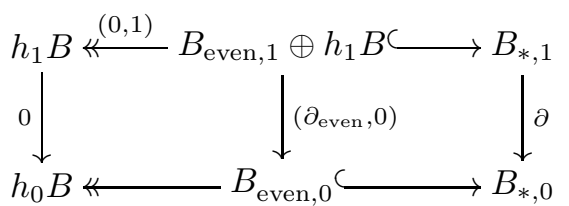

Algebras over the Eilenberg-Mac Lane spectrum $H \mathbb{Z}$ also neglect the Hopf map. Any connective $H \mathbb{Z}$-algebra is weakly equivalent to the $H \mathbb{Z}$-algebra $\mathbb{H} D_{*}$ of a differential graded algebra $D_{*}$ concentrated in non-negative dimensions, see [Shi06. The theory developed in this paper allows one to compute a small model of $\pi_{*, *}^{a d d} \mathbb{H} D_{*}$. Indeed this pair algebra is quasi-isomorphic to

$$
\bar{d}: \Sigma^{-1}\left(D_{*} / d\left(D_{*}\right)\right) \longrightarrow Z_{*} .
$$

Here $Z_{*} \subset D_{*}$ is the subring of cycles and $\bar{d}$ is induced by the differential in $D_{*}$, compare [BM02, 3.6].

The algebra of secondary $\bmod p$ cohomology operations $\mathcal{B}$, for $p$ a fixed prime, computed in Bau06, is a pair algebra. This pair algebra has proved to be useful for computations of $d_{2}$ differentials in the classical Adams spectral sequence, see BJ04. The pair algebra $\mathcal{B}$ corresponds to the coconnective version of $\pi_{*, *} R$ for $R=\operatorname{End}(H \mathbb{Z} / p)$ the endomorphism spectrum of the mod $p$ Eilenberg-Mac Lane spectrum. The coconnective theory, however, is not considered in this paper.

Remark 2.9. Given an ungraded ring $A$ and an ungraded $A$-bimodule $M$, the Shukla cohomology $S H^{3}(A, M)$ is naturally included in Mac Lane cohomology, see [JP86, BP, which is isomorphic to the topological Hochschild cohomology of the corresponding Eilenberg-Mac Lane ring spectrum, compare [PW92],

$$
S H^{3}(A, M) \hookrightarrow H M L^{3}(A, M) \cong T H H^{3}(H A, H M) .
$$

If $R$ is a connective ring spectrum neglecting $\eta$ we can consider the ungraded pair algebra $\pi_{0, *}^{a d d} R$ in the bottom degree of $\pi_{*, *}^{a d d} R$ in Theorem 2.7yielding the associated Shukla cohomology class

$$
\left\langle\pi_{0, *}^{a d d} R\right\rangle \in S H^{3}\left(\pi_{0} R, \pi_{1} R\right) .
$$


On the other hand Lazarev [Laz01] introduced the "first $k$-invariant"

$$
k_{R}^{1} \in T H H^{3}\left(H\left(\pi_{0} R\right), H\left(\pi_{1} R\right)\right)
$$

of the ring spectrum $R$. We claim that the image under (2.10) of the class $\left\langle\pi_{0, *}^{a d d} R\right\rangle$ in topological Hochschild cohomology can be identified with the first Postnikov invariant $k_{R}^{1}$ of the ring spectrum $R$.

\section{3. $E_{\infty}$-Pair algebras Associated to Commutative Ring SPeCtra}

A commutative pair algebra is a commutative monoid in $\mathbf{p m}^{\mathbb{N}}$. We need a weaker notion given by a pair algebra which is commutative up to a coherent homotopy as follows.

Definition 3.1. An $E_{\infty}$-pair algebra $C=\left(C, \smile_{1}\right)$ is a pair algebra $C=C_{*, *}$ together with a homomorphism

$$
\smile_{1}: C_{*, 0} \otimes C_{*, 0} \longrightarrow C_{*, 1}
$$

also called cup-one product such that given $x_{i} \in C_{n_{i}, 0}$ and $s \in C_{m, 1}$,

$$
\begin{aligned}
(-1)^{n_{1} \cdot n_{2}} x_{2} \cdot x_{1}+\partial\left(x_{1} \smile_{1} x_{2}\right) & =x_{1} \cdot x_{2}, \\
(-1)^{m \cdot n_{2}} x_{2} \cdot s+\partial(s) \smile_{1} x_{2} & =s \cdot x_{2}, \\
(-1)^{n_{1} \cdot n_{2}} x_{2} \smile_{1} x_{1}+x_{1} \smile_{1} x_{2} & =0, \\
\left(x_{1} \cdot x_{2}\right) \smile_{1} x_{3} & =(-1)^{n_{2} \cdot n_{3}}\left(x_{1} \smile_{1} x_{3}\right) \cdot x_{2}+x_{1} \cdot\left(x_{2} \smile_{1} x_{3}\right) .
\end{aligned}
$$

The ring $h_{0} C$ is commutative and $h_{1} C$ is an $h_{0} C$-module in a natural way.

Remark 3.2. If $\mu: C \bar{\otimes} C \rightarrow C$ denotes the multiplication on the $E_{\infty}$-pair algebra $C$ then the cup-one product consists exactly of a chain homotopy $\smile_{1}: \mu \tau_{\bar{\otimes}} \Rightarrow \mu$ which is idempotent

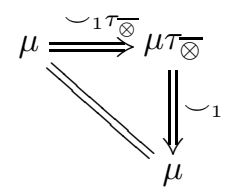

and such that the hexagon

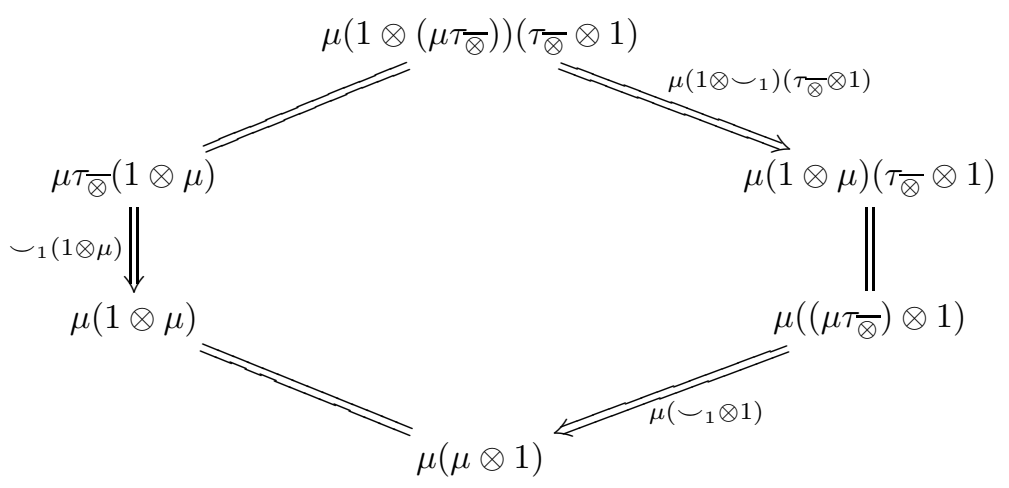

commutes. These are the usual axioms in a symmetric monoidal category, see Bor94. Indeed there is an additional diagram, called the pentagon, which should commute. In this case it commutes automatically since the product in $C$ is strictly associative.

Pair algebras carry a notion of Massey product as we saw in Definition [2.6. $E_{\infty}$-pair algebras carry in addition a notion of cup-one square. 
Definition 3.3. Let $C$ be an $E_{\infty}$-pair algebra. Given an element $a \in h_{0} C_{2 n \text {,* }}$ we define the cup-one square $S q_{1}(a)$ of $a$ in the following way. Choose a representative $\bar{a} \in C_{2 n, 0}$ of $a$. Then we have

$$
\partial\left(\bar{a} \smile_{1} \bar{a}\right) \quad=\quad 0
$$

so we can set

$$
S q_{1}(a)=\bar{a} \smile_{1} \bar{a} \in h_{1} C_{4 n, *} .
$$

One readily checks that the cup-one square construction $S q_{1}(a)$ does not depend on the choice of a representative $\bar{a}$ made for its definition.

Theorem 3.4. There is a diagram of functors

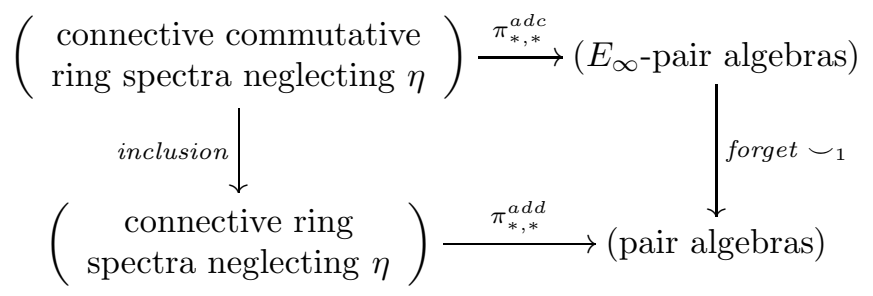

Here the lower horizontal arrow is the functor in Theorem 2.7. This diagram commutes up to natural quasi-isomorphisms as in (17.4) below. Moreover, given a connective commutative ring spectrum $Q$ the algebraic cup-one squares in $\pi_{*, *}^{a d c} Q$ coincide with the topologically-defined cup-one squares in $\pi_{*} Q$.

We prove this theorem in Section 17. It follows from Theorems 3.4 and 2.7 that Massey products in $\pi_{*, *}^{a d c} R$ coincide with Toda brackets in $R$.

Example 3.5. Let $D_{*}$ be a differential graded algebra concentrated in non-negative dimensions endowed with a $\smile_{1}$ operation consisting of a degree 1 homomorphism

$$
\smile_{1}: D_{*} \otimes D_{*} \longrightarrow D_{*}
$$

satisfying the Hirsch formulas

$$
\begin{aligned}
d\left(x_{1} \smile_{1} x_{2}\right) & =x_{1} \cdot x_{2}-(-1)^{n_{1} \cdot n_{2}} x_{2} \cdot x_{1}-d\left(x_{1}\right) \smile_{1} x_{2}-(-1)^{n_{1}} x_{1} \smile_{1} d\left(x_{2}\right), \\
\left(x_{1} \cdot x_{2}\right) \smile_{1} x_{3} & =(-1)^{n_{1}} x_{1} \cdot\left(x_{2} \smile_{1} x_{3}\right)+(-1)^{n_{2} \cdot n_{3}}\left(x_{1} \smile_{1} x_{3}\right) \cdot x_{2},
\end{aligned}
$$

compare [McC85, page 267]. Then the pair algebra

$$
\bar{d}: \Sigma^{-1}\left(D_{*} / d\left(D_{*}\right)\right) \longrightarrow Z_{*}
$$

in Example 2.8 is an $E_{\infty}$-pair algebra with cup-one product induced by $\smile_{1}$ in (3.6).

Example 3.7. Let $S_{(3)}$ be the 3-local sphere spectrum, which is a commutative ring spectrum, and let $\mathbb{Z}_{(3)}$ be the 3 -local integers. The computations in this example are based on classical results which can be found for instance in Tod62 and Rav86. Up to dimension 13 the abelian group $\pi_{n} S_{(3)}$ is given by

\begin{tabular}{|c||c|c|c|c|c|c|c|c|c|c|c|c|c|c|}
$n$ & 0 & 1 & 2 & 3 & 4 & 5 & 6 & 7 & 8 & 9 & 10 & 11 & 12 & 13 \\
\hline$\pi_{n} S_{(3)}$ & $\mathbb{Z}_{(3)}$ & 0 & 0 & $\mathbb{Z} / 3$ & 0 & 0 & 0 & $\mathbb{Z} / 3$ & 0 & 0 & $\mathbb{Z} / 3$ & $\mathbb{Z} / 9$ & 0 & $\mathbb{Z} / 3$ \\
\hline generators & 1 & - & - & $\alpha_{1}$ & - & - & - & $\alpha_{2}$ & - & - & $\beta_{1}$ & $\alpha_{3}^{\prime}$ & 0 & $\alpha_{1} \cdot \beta_{1}$
\end{tabular}

The stable homotopy ring $\pi_{*} S_{(3)}$ is a commutative $\mathbb{Z}_{(3)}$-algebra. The only product in degrees $\leq 13$ which is not indicated above is

$$
\alpha_{1} \cdot \alpha_{2}=0 .
$$


Moreover, the non-vanishing secondary homotopy operations in this range are the Toda brackets

$$
\begin{aligned}
\left\langle\alpha_{1}, 3, \alpha_{1}\right\rangle & =\alpha_{2}, \\
\left\langle\alpha_{1}, \alpha_{1}, \alpha_{1}\right\rangle & =\beta_{1}, \\
\left\langle\alpha_{1}, 3, \alpha_{2}\right\rangle & =3 \cdot \alpha_{3}^{\prime} .
\end{aligned}
$$

We are going to show in this example that the knowledge of the secondary homotopy operations gives us some information about the $E_{\infty}$-pair algebra $\pi_{*, *}^{a d c} S_{(3)}$ in degrees $\leq 12$, but it does not determine the whole structure. This proves that the algebraic object $\pi_{*, *}^{a d c} S_{(3)}$ contains more information than just the collection of classical secondary homotopy operations.

The natural morphism $\pi_{*, *}^{a d c} S_{(3)} \rightarrow \pi_{*, *}^{a d c} S_{(3)} \otimes \mathbb{Z}_{(3)}$ is a quasi-isomorphism since $\pi_{*} S_{(3)}$ is 3-local. Let us choose $a_{1}, a_{2}, b_{1}, a_{3}^{\prime} \in \pi_{*, 0} S_{(3)} \otimes \mathbb{Z}_{(3)}$ representing $\alpha_{1}, \alpha_{2}, \beta_{1}$, $\alpha_{3}^{\prime}$, respectively. Taking pull-backs, as in Section [14, we can define a quasiisomorphic $E_{\infty}$-pair algebra $C \rightarrow \pi_{*, *}^{a d c} S_{(3)} \otimes \mathbb{Z}_{(3)}$ such that $C_{*, 0}$ coincides in dimensions $\leq 12$ with the free $\mathbb{Z}_{(3)}$-algebra generated by $a_{1}, a_{2}, b_{1}, a_{3}^{\prime}$. Any submodule of a free $\mathbb{Z}_{(3)}$-module is free since $\mathbb{Z}_{(3)}$ is a principal ideal domain, therefore, since $h_{0} C \cong \pi_{*} S_{(3)}$ and $h_{1} C \cong \Sigma^{-1} \pi_{*} S_{(3)}$, the pair modules $C_{n, *}$ are given for $n \leq 12$ by

\begin{tabular}{|c||c|c||c|c|}
$n$ & $C_{n, 0}$ & generators & $C_{n, 1}$ & generators \\
\hline 0 & $\mathbb{Z}_{(3)}$ & 1 & 0 & - \\
\hline 1 & 0 & - & 0 & - \\
\hline 2 & 0 & - & $\mathbb{Z} / 3$ & $\Sigma^{-1} \alpha_{1}$ \\
\hline 3 & $\mathbb{Z}_{(3)}$ & $\mathbb{Z}_{(3)}$ & $\bar{a}_{1}$ \\
\hline 4 & 0 & - & 0 & - \\
\hline 5 & 0 & - & 0 & - \\
\hline 6 & $\mathbb{Z}_{(3)}$ & $\mathbb{Z}_{(3)} \oplus \mathbb{Z} / 3$ & $\bar{a}_{1}^{(2)}, \Sigma^{-1} \alpha_{2}$ \\
\hline 7 & $\mathbb{Z}_{(3)}$ & $a_{2}$ & 0 & $\bar{Z}_{2}$ \\
\hline 8 & 0 & - & $\mathbb{Z}_{(3)}$ & - \\
\hline 9 & $\mathbb{Z}_{(3)}$ & $a_{1}^{3} \oplus \bar{a}_{1}^{(3)}, \Sigma^{-1} \beta_{1}$ \\
\hline 10 & $\mathbb{Z}_{(3)} \oplus \mathbb{Z}_{(3)} \oplus \mathbb{Z}_{(3)}$ & $a_{1} \cdot a_{2}, a_{2} \cdot a_{1}, b_{1}$ & $\mathbb{Z}_{(3)} \oplus \mathbb{Z}_{(3)} \oplus \mathbb{Z}(3) \oplus \mathbb{Z} / 9$ & $\bar{a}_{1,2}, \bar{a}_{2,1}, b_{1}, \Sigma^{-1} \alpha_{3}^{\prime}$ \\
\hline 11 & $\mathbb{Z}_{(3)}$ & $a_{3}^{\prime}$ & $\mathbb{Z}_{(3)}$ & $\bar{a}_{3}^{\prime}$ \\
\hline 12 & $\mathbb{Z}_{(3)}$ & $a_{1}^{4}$ & $\mathbb{Z}_{(3)} \oplus \mathbb{Z} / 3$ & $\bar{a}_{1}^{(4)}, \Sigma^{-1}\left(\alpha_{1} \cdot \beta_{1}\right)$
\end{tabular}

where $\bar{a}_{1}, \bar{a}_{1}^{(2)}, \bar{a}_{2}, \bar{a}_{1}^{(3)}, \bar{a}_{1,2}, \bar{a}_{2,1}, \bar{b}_{1}, \bar{a}_{3}^{\prime}, \bar{a}_{1}^{(4)}$ are arbitrarily chosen elements satisfying

$$
\begin{aligned}
\partial\left(\bar{a}_{i}\right) & =3 \cdot a_{i}, \quad i=1,2, \\
\partial\left(\bar{a}_{1}^{(i)}\right) & =a_{1}^{i}, \quad i=2,3,4, \\
\partial\left(\bar{a}_{i, j}\right) & =a_{i} \cdot a_{j}, \quad\{i, j\}=\{1,2\}, \\
\partial\left(\bar{b}_{1}\right) & =3 \cdot b_{1}, \\
\partial\left(\bar{a}_{3}^{\prime}\right) & =9 \cdot a_{3}^{\prime} .
\end{aligned}
$$

The desuspended elements are in $h_{1} C=\operatorname{Ker} \partial$. Since $\partial$ is a $C_{*, 0}$-bimodule homomorphism

$$
\begin{aligned}
& a_{1} \cdot \bar{a}_{1}=3 \cdot \bar{a}_{1}^{(2)}+m \cdot\left(\Sigma^{-1} \alpha_{2}\right), \\
& a_{1} \cdot \bar{a}_{2}=3 \cdot \bar{a}_{1,2}+m^{\prime} \cdot\left(\Sigma^{-1} \alpha_{3}^{\prime}\right), \\
& \bar{a}_{1} \cdot a_{2}=3 \cdot \bar{a}_{1,2}+m^{\prime \prime} \cdot\left(\Sigma^{-1} \alpha_{3}^{\prime}\right),
\end{aligned}
$$

for some $m \in \mathbb{Z} / 3$ and $m^{\prime}, m^{\prime \prime} \in \mathbb{Z} / 9$, and we can choose $\bar{a}_{1}^{(3)}$ and $\bar{a}_{1}^{(4)}$ so that

$$
a_{1}^{i} \cdot \bar{a}_{1}^{(2)}=\bar{a}_{1}^{(i+2)}, \quad i=1,2 .
$$


Moreover,

$$
\begin{aligned}
\bar{a}_{1}^{(2)} \cdot a_{1}^{2} & =\bar{a}_{1}^{(2)} \cdot \partial\left(\bar{a}_{1}^{(2)}\right) \\
& =\partial\left(\bar{a}_{1}^{(2)}\right) \cdot \bar{a}_{1}^{(2)} \\
& =a_{1}^{2} \cdot \bar{a}_{1}^{(2)} \\
& =\bar{a}_{1}^{(4)} .
\end{aligned}
$$

By the laws of an $E_{\infty}$-pair algebra we see that we can choose $a_{1}^{(2)}, \bar{a}_{1,2}, \bar{a}_{2,1}$ in such a way that

$$
\begin{aligned}
& a_{1} \smile_{1} a_{1}=2 \cdot \bar{a}_{1}^{(2)}, \\
& a_{1} \smile_{1} a_{2}=\bar{a}_{2,1}+\bar{a}_{1,2},
\end{aligned}
$$

and moreover

$$
\begin{aligned}
\bar{a}_{1} \cdot a_{1} & =-a_{1} \cdot \bar{a}_{1}+a_{1} \smile_{1}\left(3 \cdot a_{1}\right) \\
& =-3 \cdot \bar{a}_{1}^{(2)}-m \cdot\left(\Sigma^{-1} \alpha_{2}\right)+6 \cdot \bar{a}_{1}^{(2)} \\
& =3 \cdot \bar{a}_{1}^{(2)}-m \cdot\left(\Sigma^{-1} \alpha_{2}\right), \\
\bar{a}_{2} \cdot a_{1} & =-a_{1} \cdot \bar{a}_{2}+a_{1} \smile_{1}\left(3 \cdot a_{2}\right) \\
& =-3 \cdot \bar{a}_{1,2}-m^{\prime} \cdot\left(\Sigma^{-1} \alpha_{3}^{\prime}\right)+3 \cdot \bar{a}_{2,1}+3 \cdot \bar{a}_{1,2} \\
& =3 \cdot \bar{a}_{2,1}-m^{\prime} \cdot\left(\Sigma^{-1} \alpha_{3}^{\prime}\right), \\
a_{2} \cdot \bar{a}_{1} & =-\bar{a}_{1} \cdot a_{2}+a_{2} \smile_{1}\left(3 \cdot a_{1}\right) \\
& =-3 \cdot \bar{a}_{1,2}-m^{\prime \prime} \cdot\left(\Sigma^{-1} \alpha_{3}^{\prime}\right)+3 \cdot \bar{a}_{2,1}+3 \cdot \bar{a}_{1,2} \\
& =3 \cdot \bar{a}_{2,1}-m^{\prime \prime} \cdot\left(\Sigma^{-1} \alpha_{3}^{\prime}\right) .
\end{aligned}
$$

The action of $a_{1}$ on $\Sigma^{-1} \alpha_{1}, \Sigma^{-1} \alpha_{2}$, yields 0 from the left and from the right, as well as the action of $a_{2}$ on $\Sigma^{-1} \alpha_{1}$, and

$$
\begin{aligned}
a_{1} \cdot\left(\Sigma^{-1} \beta_{1}\right) & =-\Sigma^{-1}\left(\alpha_{1} \cdot \beta_{1}\right) \\
& =-\Sigma^{-1}\left(\beta_{1} \cdot \alpha_{1}\right) \\
& =-\left(\Sigma^{-1} \beta_{1}\right) \cdot a_{1} .
\end{aligned}
$$

Finally the known Toda brackets yield the equalities

$$
\begin{aligned}
\Sigma^{-1} \alpha_{2} & =-\bar{a}_{1} \cdot a_{1}+a_{1} \cdot \bar{a}_{1} \\
& =-m \cdot\left(\Sigma^{-1} \alpha_{2}\right), \\
\Sigma^{-1} \beta_{1} & =-\bar{a}_{1}^{(2)} \cdot a_{1}+a_{1} \cdot \bar{a}_{1}^{(2)} \\
& =-\bar{a}_{1}^{(2)} \cdot a_{1}+\bar{a}_{1}^{(3)}, \\
3 \cdot\left(\Sigma^{-1} \alpha_{3}^{\prime}\right) & =-\bar{a}_{1} \cdot a_{2}+a_{1} \cdot \bar{a}_{2} \\
& =\left(m^{\prime}-m^{\prime \prime}\right) \cdot\left(\Sigma^{-1} \alpha_{3}^{\prime}\right) .
\end{aligned}
$$

In particular

$$
\begin{aligned}
m & =-1 \in \mathbb{Z} / 3, \\
m^{\prime \prime} & =m^{\prime}-3 \in \mathbb{Z} / 9 .
\end{aligned}
$$

The equations above determine, up to dimension 9 , the structure of $C$, and hence the structure of $\pi_{*, *}^{a d c} S_{(3)}$ up to quasi-isomorphism. In dimensions $\leq 12$ the structure is determined up to the unknown constant $m^{\prime} \in \mathbb{Z} / 9$, which can not be deduced from the secondary homotopy operations in $\pi_{*} S_{(3)}$. The reader who wants to familiarize himself with the new algebraic invariants introduced in this paper can try to compute more about $\pi_{*, *}^{a d c} S_{(3)}$ with the help of the classical calculations available in the literature. One can also try with primes $p>3$. 
We remark that the elements $\Sigma^{-1} \alpha_{1}, a_{1}, \bar{a}_{1}, a_{2}, \bar{a}_{2}, b_{1}, \bar{a}_{1,2}, \bar{b}_{1}, \Sigma^{-1} \alpha_{3}^{\prime}, a_{3}^{\prime}, \bar{a}_{3}^{\prime}$, generate $C$ as an $E_{\infty}$-pair algebra in dimensions $\leq 12$ since the rest of elements $\bar{a}_{1}^{(i)}$, $\Sigma^{-1} \alpha_{2}, \bar{a}_{2,1}, \Sigma^{-1} \beta_{1}, \Sigma^{-1}\left(\alpha_{1} \cdot \beta_{1}\right), i=2,3,4$, can be written in terms of these generators, the product and the cup-one product in $C$.

Remark 3.8. The coherence conditions in Remark 3.2 are related to the BarrattEccles operad introduced in BE74. Indeed, one can check that $E_{\infty}$-pair algebras are algebras over the following operad in pm. Let $\mathcal{B E}$ be the simplicial BarrattEccles operad and let $C_{*} \mathcal{B E}$ be the operad of chain complexes obtained by taking normalized cochains on $\mathcal{B E}$. If we truncate this operad by dividing out the subcomplexes generated by the elements in dimensions $\geq 2$ we obtain an operad $t_{\leq 2} C_{*} \mathcal{B E}$ in $\mathbf{p m}$ whose associated algebras are exactly the $E_{\infty}$-pair algebras.

Now let $A$ be an ungraded commutative ring and let $M$ be an ungraded $A$ module. We consider the category consisting of ungraded $E_{\infty}$-pair algebras $C_{*}$ with specified isomorphisms $h_{0} C_{*} \cong A$ and $h_{1} C_{*} \cong M$ and $E_{\infty}$-pair algebra morphisms over $A$ and under $M$. Then the set of components of this category is in natural bijection with the operadic cohomology group

$$
H_{t_{\leq 2} C_{*} \mathcal{B E}}^{3}(A, M)
$$

defined in BMR04, which in dimension 3 is the "commutative analogue" of Shukla cohomology, see Remark 2.5. This cohomology is likely to be related to the $\Gamma$ cohomology of Robinson and Whitehouse, compare [BMR04, 7]. Moreover, this operadic cohomology group should map to the third topological Andre-Quillen cohomology group, where the first Postnikov invariant of a connective commutative ring spectrum $R$ lives, see Bas99. In fact, if $R$ neglects the Hopf map the class of the ungraded $E_{\infty}$-pair algebra $\pi_{0, *}^{a d c} R$ should be mapped this way to the first Postnikov invariant of $R$ as in the non-commutative case, see Remark 2.9.

\section{Algebras over Commutative Ring spectra Neglecting the Hopf map}

In stable homotopy theory we have the notion of a connective algebra over a connective commutative ring spectrum $Q$, which is a connective ring spectrum $R$ with a two-sided action of $Q$. If $Q$ neglects the Hopf map then so $R$ does and by Theorems 2.7 and 3.4 the ring spectra $R$ and $Q$ give rise to a pair algebra $\pi_{*, *}^{a d d} R$ and to an $E_{\infty}$-pair algebra $\pi_{*, *}^{a d c} Q$. In this section we study the algebraic properties of the induced action of $\pi_{*, *}^{a d c} Q$ on $\pi_{*, *}^{a d d} R$, obtaining Theorem 1.5 for spectra neglecting the Hopf map as an application.

Definition 4.1. Let $C$ be an $E_{\infty}$-pair algebra. A $C$-algebra is a pair algebra $B$ together with a pair algebra morphism $u: C \rightarrow B$, called the unit, and two external cup-one product operations,

$$
\begin{array}{r}
\smile_{1}: B_{*, 0} \otimes C_{*, 0} \longrightarrow C_{*, 1}, \\
\smile_{1}: C_{*, 0} \otimes B_{*, 0} \longrightarrow C_{*, 1},
\end{array}
$$


such that given $x_{i} \in B_{n_{i}, 0}, s \in B_{m, 1}, \tilde{x}_{i} \in C_{n_{i}, 0}, \tilde{s} \in C_{m, 1}$, the following equations hold, compare Definition 3.1.

$$
\begin{aligned}
u_{1}\left(\tilde{x}_{1} \smile_{1} \tilde{x}_{2}\right) & =u_{0}\left(\tilde{x}_{1}\right) \smile_{1} \tilde{x}_{2} \\
& =\tilde{x}_{1} \smile_{1} u_{0}\left(\tilde{x}_{2}\right), \\
(-1)^{n_{1} \cdot n_{2}} x_{2} \cdot u_{0}\left(\tilde{x}_{1}\right)+\partial\left(\tilde{x}_{1} \smile_{1} x_{2}\right) & =u_{0}\left(\tilde{x}_{1}\right) \cdot x_{2}, \\
(-1)^{m \cdot n_{2}} u_{0}\left(\tilde{x}_{2}\right) \cdot s+\partial(s) \smile_{1} \tilde{x}_{2} & =s \cdot u_{0}\left(\tilde{x}_{2}\right), \\
(-1)^{m \cdot n_{2}} x_{2} \cdot u_{1}(\tilde{s})+\partial(\tilde{s}) \smile_{1} x_{2} & =u_{1}(\tilde{s}) \cdot x_{2}, \\
(-1)^{n_{1} \cdot n_{2}} x_{2} \smile_{1} \tilde{x}_{1}+\tilde{x}_{1} \smile_{1} x_{2} & =0, \\
(-1)^{n_{2} \cdot n_{3}}\left(\tilde{x}_{1} \smile_{1} x_{3}\right) \cdot u_{0}\left(\tilde{x}_{2}\right)+u_{0}\left(\tilde{x}_{1}\right) \cdot\left(\tilde{x}_{2} \smile_{1} x_{3}\right) & =\left(\tilde{x}_{1} \cdot \tilde{x}_{2}\right) \smile_{1} x_{3}, \\
(-1)^{n_{2} \cdot n_{3}}\left(x_{1} \smile_{1} \tilde{x}_{3}\right) \cdot x_{2}+x_{1} \cdot\left(x_{2} \smile_{1} \tilde{x}_{3}\right) & =\left(x_{1} \cdot x_{2}\right) \smile_{1} \tilde{x}_{3} .
\end{aligned}
$$

The ring $h_{0} B$ is an $h_{0} C$-algebra in such a way that the $h_{0} B$-bimodule $h_{1} B$ restricts to an $h_{0} C$-module.

Remark 4.9. Any $E_{\infty}$-pair algebra is an algebra over itself. Moreover, given a morphism of $E_{\infty}$-pair algebras $f: \bar{C} \rightarrow C$ and a $C$-algebra $B$ then $B$ has a $\bar{C}$ algebra structure, denoted by $f^{*} B$, with unit $u f: \bar{C} \rightarrow B$ and external cup-one products obtained by precomposing with $f$.

The main new secondary homotopy operation on algebras over $E_{\infty}$-pair algebras is introduced in the next definition. We need to recall first some basics facts on the 1-dimensional Hochschild cohomology of graded algebras.

Recall that given a commutative algebra $K$, a $K$-algebra $A$, an $A$-bimodule $M$ which is also a $K$-module, and $n \in \mathbb{Z}$, a degree $n$ derivation is a degree $n$ homomorphism of $K$-modules $d: A \rightarrow M$ such that $d(a \cdot b)=d(a) \cdot b+(-1)^{n|a|} a \cdot d(b)$. Any $m \in M$ gives rise to a degree $|m|$ derivation $d_{m}(a)=m \cdot a-(-1)^{|m||a|} a$. $m$. These derivations are called inner derivations and they form a submodule $\operatorname{Inn}_{K}(A, M) \subset \operatorname{Der}_{K}(A, M)$ of the $K$-module of all derivations. The 1-dimensional Hochschild cohomology group of $A$ with coefficients in $M$ coincides with the quotient $K$-module

$$
H H_{K}^{1}(A, M)=\operatorname{Der}_{K}(A, M) / \operatorname{Inn}_{K}(A, M) .
$$

Definition 4.10. Let $C$ be an $E_{\infty}$-pair algebra and let $B$ be a $C$-algebra. We associate to any pair $(x, y)$ with $x \in C_{0, n}$ and $y \in B_{n, 1}$ such that $\partial(y)=u_{0}(x)$ the degree $n$ derivation

defined by

$$
\theta_{(x, y)}: h_{0} B \longrightarrow h_{1} B
$$

$$
\theta_{(x, y)}(a)=-y \cdot \bar{a}+(-1)^{n|a|} \bar{a} \cdot y+x \smile_{1} \bar{a} .
$$

Here $\bar{a} \in B_{*, 0}$ is a representative of $a \in h_{0} B$. This is indeed an element in $h_{1} B$ by (4.3). Moreover, $\theta_{(x, y)}=0$ provided $B$ is an $E_{\infty}$-pair algebra and $u$ is a morphism of $E_{\infty}$-pair algebras. It follows from equations (4.4) and (4.6) that $\theta_{(x, y)}(a)$ does not depend on the choice of $\bar{a}$. In order to check the derivation property for $\theta_{(x, y)}$ one uses equations (4.8) and (4.6). It is $h_{0} C$-linear since $\theta_{(x, y)}$ vanishes in the image of $h_{0} u$. This follows from equations (4.2) and (4.5).

Proposition 4.11. Given an $E_{\infty}$-pair algebra $C$ and a $C$-algebra $B$ let $I_{C}(B)$ be the kernel of the ring homomorphism $h_{0} u: h_{0} C \rightarrow h_{0} B$. There is an $h_{0} C$-module homomorphism

$$
\theta: I_{C}(B) / I_{C}(B)^{2} \longrightarrow H H_{h_{0} C}^{1}\left(h_{0} B, h_{1} B\right)
$$

which sends an element in $I_{C}(B) / I_{C}(B)^{2}$ represented by $x \in C_{n, 0}$ to the element in Hochschild cohomology represented by a derivation $\theta_{(x, y)}$ as in Definition 10.4 for any $y \in B_{n, 1}$ with $\partial(y)=u_{0}(x)$. The homomorphism $\theta$ is natural in $B$ and in 
$C$ in the obvious way. In particular $\theta=0$ when $B$ is an $E_{\infty}$-pair algebra and $u$ is a morphism of $E_{\infty}$-pair algebras.

Proof. Using equation (4.5) one can check that the cohomology class of the derivation $\theta_{(x, y)}$ only depends on the projection of $x$ to $I_{C}(B)$. In this way one obtains a homomorphism $\theta$ from $I_{C}(B)$. Given $z \in C_{m, 0}$ representing $c \in h_{0} C$

$$
\begin{aligned}
\theta_{\left(z \cdot x, u_{0}(z) \cdot y\right)}(a)= & -u_{0}(z) \cdot y \cdot \bar{a}+(-1)^{(m+n)|a|} \bar{a} \cdot u_{0}(z) \cdot y+(z \cdot x) \smile_{1} \bar{a} \\
= & -u_{0}(z) \cdot y \cdot \bar{a}+(-1)^{n|a|} u_{0}(z) \cdot \bar{a} \cdot y \\
& -(-1)^{n|a|} \partial\left(z \smile_{1} \bar{a}\right) \cdot y+(z \cdot x) \smile_{1} \bar{a} \\
= & -u_{0}(z) \cdot y \cdot \bar{a}+(-1)^{n|a|} u_{0}(z) \cdot \bar{a} \cdot y \\
& -(-1)^{n|a|}\left(z \smile_{1} \bar{a}\right) \cdot u_{0}(x)+(z \cdot x) \smile_{1} \bar{a} \\
= & -u_{0}(z) \cdot y \cdot \bar{a}+(-1)^{n|a|} u_{0}(z) \cdot \bar{a} \cdot y+u_{0}(z) \cdot\left(x \smile_{1} \bar{a}\right) \\
= & u_{0}(z) \cdot\left(-y \cdot \bar{a}+(-1)^{n|a|} \bar{a} \cdot y+x \smile_{1} \bar{a}\right) \\
= & \left(h_{0} u\right)(c) \cdot \theta_{(x, y)}(a) .
\end{aligned}
$$

This formula shows that $\theta$ is $h_{0} C$-linear and also that $\theta\left(I_{C}(B)^{2}\right)=0$.

Algebras over commutative ring spectra neglecting the Hopf map are a source of algebras over $E_{\infty}$-pair algebras as the following theorem shows.

Theorem 4.12. Let $Q$ be a connective commutative ring spectrum neglecting the Hopf map and let $\pi_{*, *}^{a d c} Q$ be the $E_{\infty}$-pair algebra given by Theorem 3.4. There is a diagram of functors

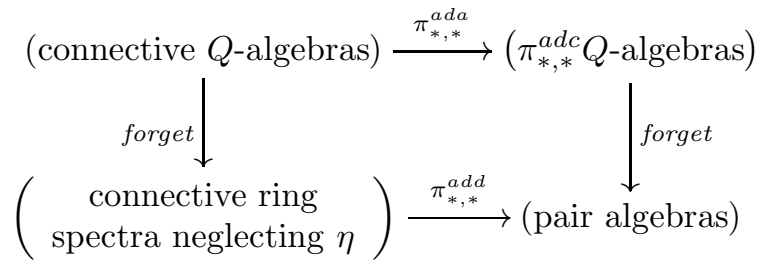

which commutes up to natural quasi-isomorphisms. Here the lower arrow is the functor in Theorem 2.7. Moreover, if $f: Q^{\prime} \rightarrow Q$ is a morphism of connective commutative ring spectra neglecting the Hopf map then the square

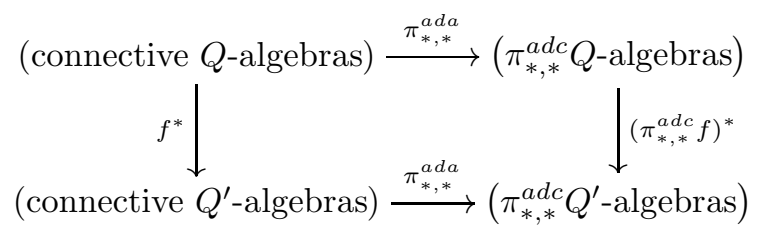

commutes up to natural quasi-isomorphisms. Furthermore, if $R$ is a connective $Q$ algebra, $a \in \pi_{m} R$, and $b \in \pi_{n} Q$ is in $I_{Q}(R)=\operatorname{Ker}\left[\pi_{*} Q \rightarrow \pi_{*} R\right] \cong I_{\pi_{* * *}^{a d c} Q}\left(\pi_{*, *}^{a d a} R\right)$, then the element $\theta_{(x, y)}(a) \in \pi_{n+m+1} R \cong\left(h_{1} \pi_{*, *}^{a d a} R\right)_{n+m}$ defined in (1.4) can be identified with $\theta_{(x, y)}(a)$ in the sense of Definition 4.10.

This theorem can be derived from Theorem 10.6 in the same way as we derive Theorem 3.4 from Theorem 9.12 in Section [17, see Remark 17.5 for details.

\section{Square groups And QuAdratic Pair modules}

We now describe quadratic generalizations of pair modules, pair algebras and $E_{\infty}$-pair algebras. The quadratic concepts are needed to achieve results as above on general ring spectra not neglecting the Hopf map. In fact, the Hopf map requires the use of quadratic structure as developed in BP99, BJP05. In this section we 
quickly recall the basic quadratic algebra which is needed in this paper. We do not recall, for example, the definition of the quadratic tensor product, but we later give explicit definitions of monoids and modules in the monoidal category of quadratic pair modules, see Definitions 6.1 and 7.1. We relate the quadratic concepts to the additive (non-quadratic) situation which was used in the previous sections.

A square group $X$ is a diagram

$$
X=\left(X_{e} \underset{H}{\stackrel{P}{\leftrightarrows}} X_{e e}\right)
$$

where $X_{e}$ is a group with an additively written group law, $X_{e e}$ is an abelian group, $P$ is a homomorphism, $H$ is a quadratic map, i.e. a function such that the cross effect

$$
(a \mid b)_{H}=H(a+b)-H(b)-H(a), a, b \in X_{e},
$$

is bilinear, and the following relations are satisfied, $x, y \in X_{e e}$,

$$
\begin{aligned}
(P x \mid b)_{H} & =(a \mid P y)=0, \\
P(a \mid b)_{H} & =-a-b+a+b, \\
P H P(x) & =P(x)+P(x) .
\end{aligned}
$$

In particular $X_{e}$ is a group of nilpotency class 2 and $P$ maps $X_{e e}$ to the center of $X_{e}$, so its image is a normal subgroup. Let $\mathbf{S G}$ be the category of square groups where a morphism of square groups $f: X \rightarrow Y$ is given by homomorphisms $f_{e}: X_{e} \rightarrow Y_{e}$, $f_{e e}: X_{e e} \rightarrow Y_{e e}$, commuting with $P$ and $H$. An abelian group is identified with a square group $X$ with $X_{e e}=0$. In this way we have a full inclusion $\mathbf{A b} \subset \mathbf{S G}$ of the category of abelian groups into a category of square groups.

For any square group $X$ the function

$$
T=H P-1: X_{e e} \longrightarrow X_{e e}
$$

is an involution, i.e. a homomorphism with $T^{2}=1$. Moreover,

$$
\Delta: X_{e} \longrightarrow X_{e e}: x \mapsto(x \mid x)_{H}-H(x)+T H(x)
$$

is a homomorphism which satisfies $T \Delta=-\Delta$. The cross effect induces a homomorphism

$$
(-\mid-)_{H}: \otimes^{2} \text { Coker } P \longrightarrow X_{e e},
$$

where $\otimes^{2} A=A \otimes A$ is the tensor square of an abelian group. We say that $X$ is good if this homomorphism is an isomorphism.

As an example of square group we can consider

$$
\mathbb{Z}_{n i l}=(\mathbb{Z} \underset{H}{\stackrel{P}{\leftrightarrows}} \mathbb{Z})
$$

with $P=0$ and $H(n)=\left(\begin{array}{l}n \\ 2\end{array}\right)=\frac{n(n-1)}{2}$.

For a pointed set $E$ with base-point $* \in E$ let $\mathbb{Z}[E]$ be the free abelian group with basis $E-\{*\}$, and let $\langle E\rangle_{n i l}$ be the quotient of the free group $\langle E\rangle$ with basis $E-\{*\}$ by triple commutators. Both $\mathbb{Z}[-]$ and $\langle-\rangle_{n i l}$ are functors on the category of pointed sets and we have the natural abelianization homomorphism $\langle E\rangle_{\text {nil }} \rightarrow \mathbb{Z}[E]$ which is supressed from notation.

There is a square group

$$
\mathbb{Z}_{n i l}[E]=\left(\langle E\rangle_{n i l} \underset{H}{\stackrel{P}{\leftrightarrows}} \otimes^{2} \mathbb{Z}[E]\right),
$$

where $P(a \otimes b)=-b-a+b+a, H(e)=0$ for any $e \in E$ and $(s \mid t)_{H}=t \otimes s$ so that $\mathbb{Z}_{n i l}\left[S^{0}\right]=\mathbb{Z}_{\text {nil }}$. These are canonical examples of good square groups. 
A quadratic pair module $C$ is a morphism $\partial: C_{(1)} \rightarrow C_{(0)}$ between square groups

$$
\begin{aligned}
& C_{(0)}=\left(C_{0} \underset{H}{\stackrel{P_{0}}{\leftrightarrows}} C_{e e}\right), \\
& C_{(1)}=\left(C_{1} \underset{H_{1}}{\stackrel{P}{\leftrightarrows}} C_{e e}\right),
\end{aligned}
$$

such that $\partial_{e e}=1: C_{e e} \rightarrow C_{e e}$ is the identity homomorphism. Hence $C$ is completely determined by the diagram

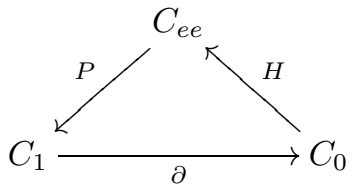

where $\partial=\partial_{e}, H_{1}=H \partial$ and $P_{0}=\partial P$. We say that $C$ is $0-\operatorname{good}$ if $C_{(0)}$ is a good square group. Moreover, $C$ is 0 -free if $C_{(0)}$ is of the form (5.1).

Morphisms of quadratic pair modules $f: C \rightarrow D$ are given by group homomorphisms $f_{0}: C_{0} \rightarrow D_{0}, f_{1}: C_{1} \rightarrow D_{1}, f_{e e}: C_{e e} \rightarrow D_{e e}$, commuting with $H, P$ and $\partial$ in (5.2). They form a category denoted by qpm. A quadratic pair module with $C_{e e}=0$ is the same as a pair module. In this way the category pm of pair modules is regarded as a full subcategory $\mathbf{p m} \subset \mathbf{q p m}$. We write $\mathbf{q p m}_{H}$ for the full subcategory of 0-good quadratic pair modules.

We have functors

$$
h_{0}, h_{1}: \mathbf{q p m} \longrightarrow \mathbf{A b}
$$

defined by $h_{0} C=$ Coker $\partial$ and $h_{1} C=\operatorname{Ker} \partial$ as in (2.1). A quasi-isomorphism of quadratic pair modules is a morphism inducing isomorphisms on $h_{0}$ and $h_{1}$.

Remark 5.3. As shown in BJP05, the category SG is a symmetric monoidal category with the tensor product $X \odot Y$ of square groups and with unit object $\mathbb{Z}_{n i l}$. Moreover, there is a symmetric monoidal structure $\odot$ on qpm defined by using the tensor product of square groups, see [BM07]. The unit object for the monoidal structure in qpm is

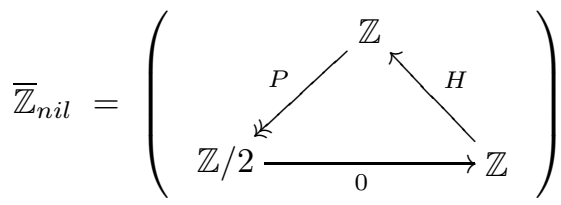

where $P$ is the non-trivial homomorphism and $H(n)=\left(\begin{array}{l}n \\ 2\end{array}\right)$ as in (5.1).

The functors $h_{0}$ and $h_{1}$ also admit pairings as in (2.2) and (2.3), so they assemble to a lax symmetric monoidal functor from $\mathbf{q p m}$ to the category of $\mathbb{N}$-graded abelian groups. This implies, since $h_{1} \overline{\mathbb{Z}}_{n i l}=\mathbb{Z} / 2$, that the unit isomorphism $C \odot \overline{\mathbb{Z}}_{n i l} \cong C$ induces a natural homomorphism

$$
k: h_{0} C \otimes \mathbb{Z} / 2 \longrightarrow h_{1} C
$$

which, in fact, is given by the formula $k(x)=P(x \mid x)_{H}$. This homomorphism, termed the $k$-invariant of $C$, corresponds to the action of the Hopf map in homotopy groups, see Propositions 13.9 and 15.4 below.

The full inclusion $\mathbf{A b} \subset \mathbf{S G}$ above admits a reflection given by

$$
(-)^{\text {add }}: \mathbf{S G} \longrightarrow \mathbf{A b}, X^{\text {add }}=\text { Coker } P \text {. }
$$

The full inclusion $\mathbf{p m} \subset \mathbf{q p m}$ also admits a reflection functor

$$
(-)^{\text {add }}: \mathbf{q p m} \longrightarrow \mathbf{p m}: C \mapsto C^{\text {add }}
$$


which sends $\partial: C_{(1)} \rightarrow C_{(0)}$ to the abelian group homomorphism

$$
\partial^{a d d}: C_{(1)}^{a d d}=\text { Coker } P \longrightarrow \text { Coker } \partial P=C_{(0)}^{a d d}
$$

induced by $\partial: C_{1} \rightarrow C_{0}$.

The natural projection $C \rightarrow C^{a d d}$, which is the unit of the reflection (5.6), induces an isomorphism on $h_{1}$ but not necessarily on $h_{0}$. For $h_{0}$ we have the following result.

Proposition 5.7. Let $C$ be a 0 -free quadratic pair module. The natural projection $C \rightarrow C^{\text {add }}$ is a quasi-isomorphism if and only if the $k$-invariant of $C$, as defined in (5.4), is trivial.

Proof. The $k$-invariant is invariant under quasi-isomorphisms, and pair modules have obviously trivial $k$-invariant, therefore the "only if" part follows.

Suppose now that $C$ is a 0 -free quadratic pair module with trivial $k$-invariant and $C_{(0)}=\mathbb{Z}_{n i l}[E]$. Then the homomorphism

$$
P: \otimes^{2} \mathbb{Z}[E] \longrightarrow C_{1}
$$

factors through the exterior square by

$$
\tilde{P}: \wedge^{2} \mathbb{Z}[E] \longrightarrow C_{1} .
$$

The composite $\partial \tilde{P}: \wedge^{2} \mathbb{Z}[E] \rightarrow\langle E\rangle_{n i l}$ is known to be an injection with cokernel $\mathbb{Z}[E]$, therefore $\tilde{P}$ is also injective. This implies that we have a commutative diagram with exact rows

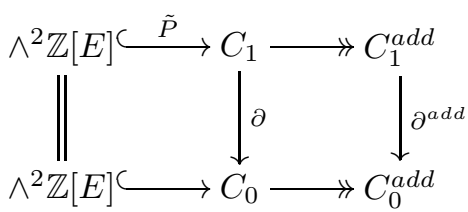

hence the proposition follows from the "snake lemma".

Remark 5.8. The inclusion $\mathbf{A b} \subset \mathbf{S G}$ strictly preserves the tensor product. Moreover, $\mathbf{A b} \subset \mathbf{S G}$ is a lax symmetric monoidal functor with lax symmetric monoidal structure given on units by the square group morphism $\mathbb{Z}_{n i l} \rightarrow \mathbb{Z}$ which is the identity on the $e$-level. The reflection (5.5) is strict symmetric monoidal since it preserves the tensor product and the unit $\mathbb{Z}_{\text {nil }}^{\text {add }}=\mathbb{Z}$.

Similarly the inclusion pm $\subset \mathbf{q p m}$ is lax symmetric monoidal. It is strictly compatible with the tensor product, and the structure morphism $\overline{\mathbb{Z}}_{n i l} \rightarrow \mathbb{Z}$ between the units is the identity on the 0 -level. As in the case of square groups the reflection functor (5.6) is strict symmetric monoidal.

Remark 5.9. A permutative category is a small category $\mathbf{P}$ with a symmetric monoidal structure $\oplus$ with unit object 0 which is strictly associative

$$
(A \oplus B) \oplus C=A \oplus(B \oplus C),
$$

and strictly unital

$$
\begin{aligned}
A \oplus 0 & =A \\
& =0 \oplus A,
\end{aligned}
$$

but not necessarily strictly commutative, i.e. the isomorphism

$$
\tau_{\oplus}: A \oplus B \cong B \oplus A .
$$

needs not be the indentity.

A quadratic pair module $C$ yields a permutative category $\mathbf{P} C$ with object set $C_{0}$. The morphism set is the semidirect product $C_{0} \ltimes C_{1}$, where $C_{0}$ acts on the right of $C_{1}$ by the formula $c_{1}^{c_{0}}=c_{1}+P\left(\partial\left(c_{1}\right) \mid c_{0}\right)_{H}$. An element $\left(c_{0}, c_{1}\right) \in C_{0} \ltimes C_{1}$ 
is a morphism $\left(c_{0}, c_{1}\right): c_{0} \rightarrow c_{0}+\partial\left(c_{1}\right)$. The composition in $\mathbf{P} C$ is determined by the sum in $C_{0} \ltimes C_{1}$. Moreover, the monoidal structure $\oplus$ is also defined by the sum in $C_{0}$ and $C_{0} \ltimes C_{1}$, that is $A \oplus B=A+B$. The symmetry isomorphism is

$$
\tau_{\oplus}=\left(A+B, P(B \mid A)_{H}\right): A \oplus B \cong B \oplus A
$$

Notice that $\mathbf{P} C$ is indeed a groupoid.

\section{QuAdratic PAIR ALGEBRAS ASSOCIATED TO RING SPECTRA}

In this section we give an explicit description of quadratic pair algebras. We also define Massey products for quadratic pair algebras and show that they correspond to Toda brackets.

Definition 6.1. A quadratic pair algebra $B$ is an $\mathbb{N}$-graded quadratic pair module $\left\{B_{n, *}, n \in \mathbb{N}\right\}$, together with multiplications, $n, m \in \mathbb{N}$,

$$
\begin{aligned}
& B_{n, 0} \times B_{m, 0} \longrightarrow B_{n+m, 0}, \\
& B_{n, 0} \times B_{m, 1} \longrightarrow B_{n+m, 1} \text {, } \\
& B_{n, 1} \times B_{m, 0} \longrightarrow B_{n+m, 1} \text {, } \\
& B_{n, e e} \times B_{m, e e} \stackrel{\bullet}{\longrightarrow} B_{n+m, e e},
\end{aligned}
$$

and an element $1 \in B_{0,0}$ with $H(1)=0$ which is a (two-sided) unit for the first three multiplications and such that $(1 \mid 1)_{H} \in B_{0, e e}$ is a (two-sided) unit for the fourth multiplication. These multiplications are associative in all possible ways. Moreover, the following lists of equations are satisfied for $x, x_{i} \in B_{*, 0}, s, s_{i} \in B_{*, 1}$ and $a_{i} \in B_{*, e e}$. The multiplications · are always right linear

$$
\begin{aligned}
x_{1} \cdot\left(x_{2}+x_{3}\right) & =x_{1} \cdot x_{2}+x_{1} \cdot x_{3}, \\
x \cdot\left(s_{1}+s_{2}\right) & =x \cdot s_{1}+x \cdot s_{2}, \\
s \cdot\left(x_{1}+x_{2}\right) & =s \cdot x_{1}+s \cdot x_{2}, \\
a_{1} \cdot\left(a_{2}+a_{3}\right) & =a_{1} \cdot a_{2}+a_{1} \cdot a_{3} .
\end{aligned}
$$

The multiplications $\cdot$ satisfy the following left distributivity laws

$$
\begin{aligned}
\left(x_{1}+x_{2}\right) \cdot x_{3} & =x_{1} \cdot x_{3}+x_{2} \cdot x_{3}+\partial P\left(\left(x_{2} \mid x_{1}\right)_{H} \cdot H\left(x_{3}\right)\right), \\
\left(x_{1}+x_{2}\right) \cdot s & =x_{1} \cdot s+x_{2} \cdot s+P\left(\left(x_{2} \mid x_{1}\right)_{H} \cdot H \partial(s)\right), \\
\left(s_{1}+s_{2}\right) \cdot x & =s_{1} \cdot x+s_{2} \cdot x+P\left(\left(\partial\left(s_{2}\right) \mid \partial\left(s_{1}\right)\right)_{H} \cdot H(x)\right), \\
\left(a_{1}+a_{2}\right) \cdot a_{3} & =a_{1} \cdot a_{3}+a_{2} \cdot a_{3} .
\end{aligned}
$$

The homomorphisms $\partial$ are compatible with the multiplications · in the following sense

$$
\begin{aligned}
\partial(x \cdot s) & =x \cdot \partial(s), \\
\partial(s \cdot x) & =\partial(s) \cdot x, \\
\partial\left(s_{1}\right) \cdot s_{2} & =s_{1} \cdot \partial\left(s_{2}\right) .
\end{aligned}
$$

And finally, we have compatibility conditions for the multiplications $\cdot$ and the maps $P, H, \Delta$, and $(-\mid-)_{H}$,

$$
\begin{aligned}
P\left((x \mid x)_{H} \cdot a\right) & =x \cdot P(a), \\
P(a \cdot \Delta(x)) & =P(a) \cdot x, \\
H\left(x_{1} \cdot x_{2}\right) & =\left(x_{1} \mid x_{1}\right)_{H} \cdot H\left(x_{2}\right)+H\left(x_{1}\right) \cdot \Delta\left(x_{2}\right), \\
H \partial P\left(a_{1} \cdot a_{2}\right) & =H \partial P\left(a_{1}\right) \cdot a_{2}+a_{1} \cdot H \partial P\left(a_{2}\right)-H \partial P\left(a_{1}\right) \cdot H \partial P\left(a_{2}\right), \\
\left(x_{1} \cdot x_{2} \mid x_{3} \cdot x_{4}\right)_{H} & =\left(x_{1} \mid x_{3}\right)_{H} \cdot\left(x_{2} \mid x_{4}\right)_{H} .
\end{aligned}
$$


Remark 6.2. In [BM07] we define the monoidal category $\mathbf{q p m}_{H}^{\mathbb{N}}$ of $\mathbb{N}$-graded 0-good quadratic pair modules. A quadratic pair algebra $B$ which is 0 -good is the same as a monoid in $\mathbf{q p m}_{H}^{\mathbb{N}}$. The product $a_{i} \cdot b_{j}$ of two elements in levels $i, j \in\{0,1\}$ with $i+j \leq 1$ is given by the image of the right linear generator $a_{i} @ b_{j}$ in the tensor product of square groups under the structure morphism $B \odot B \rightarrow B$ of the monoid $B$. If $i=j=e e$ then $a_{i} \cdot b_{j}$ is the image of $a_{i} \otimes b_{j} \in(B \odot B)_{e e}=B_{*, e e} \otimes B_{*, e e}$. To see this requires a technical computation which only uses the formulas for the tensor products of square groups and quadratic pair modules in BJP06, BM07. We leave it to the reader.

If $B$ is a quadratic pair algebra then $h_{0} B$ is an $\mathbb{N}$-graded ring and $h_{1} B$ is an $h_{0} B$-bimodule in a natural way. Massey products are defined for quadratic pair algebras as in Definition 2.6 above.

Definition 6.3. Let $B$ be a quadratic pair algebra. Given elements $a, b, c \in h_{0} B$, of degree $p, q, r \in \mathbb{N}$ with $a b=0$ and $b c=0$ the Massey product is the subset

$$
\langle a, b, c\rangle \subset h_{1} B_{p+q+r},
$$

which is a coset of the subgroup

$$
\left(h_{1} B_{p+q}\right) c+a\left(h_{1} B_{q+r}\right),
$$

defined as follows. Given $\bar{a} \in B_{p, 0}, \bar{b} \in B_{q, 0}, \bar{c} \in B_{r, 0}$ representing $a, b, c$, there exist $e \in B_{p+q, 1}, f \in B_{q+r, 1}$ such that $\partial(e)=\bar{a} \cdot \bar{b}, \partial(f)=\bar{b} \cdot \bar{c}$ and one can easily check that

$$
-e \cdot \bar{c}+\bar{a} \cdot f \in h_{1} B_{p+q+r} \subset B_{p+q+r, 1} .
$$

The coset $\langle a, b, c\rangle \subset h_{1} B_{p+q+r}$ coincides with the set of elements obtained in this way for all different choices of $\bar{a}, \bar{b}, \bar{c}, e$ and $f$.

The next result identifies Toda brackets for ring spectra with Massey products for quadratic pair algebras.

Theorem 6.4. There is a functor

$$
\pi_{*, *}: \text { (connective ring spectra) } \longrightarrow \text { (quadratic pair algebras) }
$$

together with natural isomorphisms

$$
\begin{aligned}
& h_{0} \pi_{*, *} R \cong \pi_{*} R, \text { of rings, } \\
& h_{1} \pi_{*, *} R \cong \Sigma^{-1} \pi_{*} R, \text { of bimodules },
\end{aligned}
$$

such that the Massey products in $\pi_{*, *} R$ coincide with the Toda brackets in $\pi_{*} R$. Moreover, using the isomorphisms as identifications the algebraically-defined $k$ invariant of the quadratic pair algebra $\pi_{*, *} R$ in (5.4),

$$
k: \pi_{*} R \otimes \mathbb{Z} / 2 \longrightarrow \Sigma^{-1} \pi_{*} R,
$$

coincides with the multiplication by $\eta$ where $\eta$ is the stable Hopf map.

We prove this theorem in Section 16

Example 6.5. Waldhausen defined in Wal78 the $K$-theory spectrum $K \mathbf{W}$ of a category $\mathbf{W}$ with cofibrations and weak equivalences. This spectrum is a symmetric spectrum, see GH99]. Moreover, if $\mathbf{W}$ is a strict monoidal category with biexact tensor product then $K \mathbf{W}$ is a ring spectrum. In [MT06] we give a small algebraic model for the quadratic pair algebra $\pi_{0, *} K \mathbf{W}$ which is generated just by the objects, weak equivalences, and cofiber sequences in $\mathbf{W}$.

The next result is an illustrating example for computations in a quadratic pair algebra. 
Proposition 6.6. Let $B$ be a quadratic pair algebra. Given $\alpha \in h_{0} B_{n, *}$ with $2 \alpha=0$ the Massey product $\langle 2, \alpha, 2\rangle$ is defined and the $k$-invariant satisfies

$$
k(\alpha)=P(\alpha \mid \alpha)_{H} \in\langle 2, \alpha, 2\rangle .
$$

Proof. Let $a \in B_{n, 0}$ be a representative of $\alpha$. We choose $1+1 \in B_{0,0}$ as a representative of $2 \in h_{0} B_{0, *}$. In order to construct an element in the Massey product $\langle 2, \alpha, 2\rangle$ we choose $b \in B_{n, 1}$ such that $\partial(b)=a(1+1)=a+a$. Notice that

$$
\begin{aligned}
\partial(b+P H(a)) & =a+a+\partial P H(a) \\
& =1 \cdot a+1 \cdot a+\partial P\left((1 \mid 1)_{H} H(a)\right) \\
& =(1+1) a .
\end{aligned}
$$

Here we use the axioms of a quadratic pair algebra. These equations show that the following element is in the Massey product $\langle 2, \alpha, 2\rangle$

$$
\begin{aligned}
-(b+P H(a))(1+1)+(1+1) b= & -(b+P H(a)+b+P H(a)) \\
& +b+b+P H(a+a) \\
= & -P H(a)-b-P H(a)-b \\
& +b+b+P H(a+a) \\
= & -P H(a)-P H(a)+P H(a+a) \\
= & P(a \mid a)_{H} .
\end{aligned}
$$

Here we use the laws of a quadratic pair algebra together with the fact that $P$ is central in any quadratic pair module.

It is a result of Toda that for the sphere spectrum $S$ and $\alpha \in \pi_{*} S$ with $2 \alpha=0$ one has $\alpha \eta \in\langle 2, \alpha, 2\rangle$ for $\eta$ the stable Hopf map. The same result for some cobordism spectra can be found in [Ale72. In fact Proposition 6.6 together with Theorem 6.4 generalizes this result for all connective ring spectra and shows:

Corollary 6.7. For any connective ring spectrum and $\alpha \in \pi_{*} R$ with $2 \alpha=0$ we have $\alpha \eta \in\langle 2, \alpha, 2\rangle$.

Remark 6.8. Given an ungraded ring $A$ and an ungraded $A$-bimodule $M$ consider the category consisting of ungraded quadratic pair algebras $B$ with specified isomorphisms $h_{0} B \cong A$ and $h_{1} B \cong M$ such that any element in $A$ is the image of some $x \in B_{0}$ with $H(x)=0$. Morphisms are quadratic pair algebra morphisms over $A$ and under $M$. It is proved in [BJP06 that the set of connected components of this category is in natural bijection with the 3-dimensional Mac Lane cohomology group

$$
H M L^{3}(A, M) \text {. }
$$

As we mention in Remark 2.9 this cohomology group coincides with topological Hochschild cohomology of the corresponding Eilenberg-Mac Lane ring spectrum

$$
T H H^{3}(H A, H M) \text {. }
$$

For $R$ a connective ring spectrum the Mac Lane cohomology class of the ungraded quadratic pair algebra $\pi_{0, *} R$

$$
\left\langle\pi_{0, *} R\right\rangle \in H M L^{3}\left(\pi_{0} R, \pi_{1} R\right)
$$

corresponds in $T H H$ to the first Postnikov invariant of the ring spectrum $R$, as studied in Laz01. Compare Remarks 2.5 and 2.9.

Remark 6.9. Given an ungraded quadratic pair algebra $B$ the permutative category $\mathbf{P} B$ is actually a ring category, i.e. it has an additional monoidal structure $\otimes$ 
satisfying the axioms of [EM05, 3.3]. The additional monoidal structure $\otimes$ is given by the product - on the set of objects, which is $B_{0}$. On morphisms it is given by

$$
\begin{aligned}
\left(x_{0}, s_{0}\right) \otimes\left(x_{1}, s_{1}\right) & =\left(x_{0} \cdot x_{1}, s_{0} \cdot \partial\left(s_{1}\right)\right) \\
& =\left(x_{0} \cdot x_{1}, \partial\left(s_{0}\right) \cdot s_{1}\right) .
\end{aligned}
$$

The monoidal structure $\otimes$ is strictly right distributive

$$
\left(x_{1} \oplus x_{2}\right) \otimes\left(x_{1} \oplus x_{3}\right)=x_{1} \otimes\left(x_{2} \oplus x_{3}\right),
$$

and there is a natural left distributivity isomorphism

$$
\left(x_{1} \cdot x_{3}+x_{2} \cdot x_{3}, P\left(\left(x_{2} \mid x_{1}\right)_{H} \cdot H\left(x_{3}\right)\right)\right):\left(x_{1} \otimes x_{3}\right) \oplus\left(x_{2} \otimes x_{3}\right) \longrightarrow\left(x_{1} \oplus x_{2}\right) \otimes x_{3} .
$$

\section{MODUles OVER QUADRATIC PAIR ALGEBRAS AND MODULE SPECTRA}

In this section we explicitly describe modules over quadratic pair algebras and we show their connection with modules over ring spectra.

Definition 7.1. Let $B$ be a quadratic pair algebra. A right $B$-module $M$ is an $\mathbb{N}$-graded quadratic pair module together with multiplications, $n, m \geq 0$,

$$
\begin{aligned}
& M_{n, 0} \times B_{m, 0} \longrightarrow M_{n+m, 0}, \\
& M_{n, 0} \times B_{m, 1} \longrightarrow M_{n+m, 1} \text {, } \\
& M_{n, 1} \times B_{m, 0} \longrightarrow M_{n+m, 1}, \\
& M_{n, e e} \times B_{m, e e} \longrightarrow M_{n+m, e e} .
\end{aligned}
$$

These multiplications are associative with respect to the multiplications in $B$. Moreover, $1 \in B_{0,0}$ acts trivially on $M_{*, 0}$ and $M_{*, 1}$, and $(1 \mid 1)_{H} \in B_{0, e e}$ acts trivially on $M_{*, e e}$. Furthermore, all equations in Definition 6.1 hold if we replace the elements on the left of any multiplication · by elements in $M$.

Notice that $h_{0} M$ and $h_{1} M$ are naturally right $h_{0} B$-modules, and there is a natural right $h_{0} B$-module morphism

$$
\cdot: h_{0} M \otimes_{h_{0} B} h_{1} B \rightarrow h_{1} M .
$$

This morphism extends the $k$-invariant of $M$ since $k(x)=x \cdot P(1 \mid 1)_{H}$ for $x \in h_{0} M$. Moreover, given $a \in h_{0} M$ and $b, c \in h_{0} B$ of degree $p, q, r$, such that $a b=0$ and $b c=0$ there is defined as in Definition 6.3 a Massey product

$$
\langle a, b, c\rangle \subset h_{1} M_{p+q+r}
$$

which is a coset of

$$
\left(h_{1} M_{p+q}\right) c+a\left(h_{1} B_{q+r}\right) .
$$

Remark 7.3. In BM07] we define the monoidal category $\mathbf{q p m}_{H}^{\mathbb{N}}$ of $\mathbb{N}$-graded 0-good quadratic pair modules. For $B$ a 0 -good quadratic pair algebra the 0-good right $B$-modules are exactly the right modules over the monoid $B$ in $\mathbf{q p m}_{H}^{\mathbb{N}}$, compare Remark 6.2

Recall that for an $\mathbb{N}$-graded ring $A$ the desuspension $\Sigma^{-1} M$ of a right $A$-module $M$ is $\left(\Sigma^{-1} M\right)_{n}=M_{n+1}, n \geq 0$, with the right $A$-module structure defined by the formula $\left(\Sigma^{-1} m\right) \cdot a=\Sigma^{-1}(m \cdot a)$.

In the following theorem we consider modules over quadratic pair algebras associated to right modules over a ring spectrum.

Theorem 7.4. Let $R$ be a connective ring spectrum. There is a functor

$$
\left.\left.\pi_{*, *}: \text { (connective right } R \text {-modules }\right) \longrightarrow \text { (right }\left(\pi_{*, *} R\right) \text {-modules }\right) \text {. }
$$


Here the quadratic pair algebra $\pi_{*, *} R$ is obtained by using the functor in Theorem 6.4. Moreover, if we use the first isomorphism in Theorem 6.4 as an identification then for any right $R$-module $M$ there are natural isomorphisms of right $\pi_{*} R$-modules

$$
\begin{aligned}
h_{0} \pi_{*, *} M \cong \pi_{*} M, \\
h_{1} \pi_{*, *} M \cong \Sigma^{-1} \pi_{*} M .
\end{aligned}
$$

Using these isomorphisms and the isomorphisms in Theorem 6.4 as identifications the algebraically-defined homomorphism (7.2) associated to $\pi_{*, *} M$

$$
\cdot: \pi_{*} M \otimes_{\pi_{*} R} \Sigma^{-1} \pi_{*} R \longrightarrow \Sigma^{-1} \pi_{*} M
$$

is defined by the right right $\pi_{*} R$-module structure of $\pi_{*} M$ according to the formula

$$
m \cdot\left(\Sigma^{-1} a\right)=(-1)^{|m|} \Sigma^{-1}(m a) .
$$

In particular the $k$-invariant of $\pi_{*, *} M$ coincides with the multiplication by the stable Hopf map $\eta$. Furthermore, Massey products in $\pi_{*, *} M$ coincide with Toda brackets in $\pi_{*} M$.

We prove this theorem in Section 16.

\section{Sign GROUP ACTIONS ON QUADRATIC PAIR MOdULES}

For the definition of an $E_{\infty}$-quadratic pair algebra below we need an action of the symmetric track groups $\operatorname{Sym}_{\square}(n)$ on a quadratic pair module. For this we recall the notion of sign group and action of a sign group from [BM06c, BM07].

Definition 8.1. A sign group is a diagram in the category of groups

$$
\{ \pm 1\} \stackrel{\imath}{\hookrightarrow} G_{\square} \stackrel{\delta}{\rightarrow} G \stackrel{\varepsilon}{\longrightarrow}\{ \pm 1\}
$$

where the first two morphisms form an extension. By abuse of notation we denote this sign group just by $G_{\square}$. The group law of the groups defining a sign group is denoted multiplicatively.

Sign groups as above were introduced in BM06c]. The main examples are the symmetric track groups $\operatorname{Sym}_{\square}(n)$ associated to the sign homomorphism of the symmetric groups

$$
\operatorname{sign}: \operatorname{Sym}(n) \rightarrow\{ \pm 1\} \text {. }
$$

The group $\operatorname{Sym}_{\square}(n)$ has a presentation with generators $\omega, t_{i}, 1 \leq i \leq n-1$, and relations

$$
\begin{aligned}
t_{1}^{2} & =1 \text { for } 1 \leq i \leq n-1, \\
\left(t_{i} t_{i+1}\right)^{3} & =1 \text { for } 1 \leq i \leq n-2, \\
\omega^{2} & =1 \\
t_{i} \omega & =\omega t_{i} \text { for } 1 \leq i \leq n-1, \\
t_{i} t_{j} & =\omega t_{j} t_{i} \text { for } 1 \leq i<j-1 \leq n-1 .
\end{aligned}
$$

Moreover, the structure of sign groups is given by $\imath(-1)=\omega, \delta(\omega)=0$, and $\delta\left(t_{i}\right)=(i i+1)$, the permutation exchanging $i$ and $i+1$ in $\{1, \ldots, n\}$.

Definition 8.2. A sign group $G_{\square}$ acts on the right of a quadratic pair module $C$ if $G$ acts on the right of $C$ by morphisms $g^{*}: C \rightarrow C, g \in G$, in qpm, and there is a bracket

$$
\langle-,-\rangle=\langle-,-\rangle_{G}: C_{0} \times G_{\square} \longrightarrow C_{1}
$$

satisfying the following properties, $x, y \in C_{0}, z \in C_{1}, s, t \in G_{\square}$.

(1) $\langle x+y, t\rangle=\langle x, t\rangle+\langle y, t\rangle+P\left(-\delta(t)^{*}(x)+\varepsilon \delta(t) x \mid \delta(t)^{*}(y)\right)_{H}$,

(2) $\varepsilon \delta(t) x+\left(\begin{array}{c}\varepsilon \delta(t) \\ 2\end{array}\right) \partial P H(x)=\delta(t)^{*}(x)+\partial\langle x, t\rangle$, 
(3) $\varepsilon \delta(t) z+\left(\begin{array}{c}\varepsilon \delta(t) \\ 2\end{array}\right) P H \partial(x)=\delta(t)^{*}(z)+\langle\partial(z), t\rangle$,

(4) $\langle x, s \cdot t\rangle=\left\langle\delta(s)^{*}(x), t\right\rangle+\left\langle\varepsilon \delta(t) x+\left(\begin{array}{c}\varepsilon \delta(t) \\ 2\end{array}\right) \partial P H(x), s\right\rangle$,

(5) for the element $\omega=\imath(-1) \in G_{\square}$ we have the $\omega$-formula:

$$
\langle x, \omega\rangle=P(x \mid x)_{H} .
$$

Notice that the $\omega$-formula corresponds to the $k$-invariant in (5.4).

Here $\varepsilon \delta(t)$ can take the value -1 . In this case $(-1) x$ means $-x \in C_{0}$, and $(-1) z=$ $-z \in C_{1}$.

Given a sign group $G_{\square}$ the "group ring" $A\left(G_{\square}\right)$ is the ungraded quadratic pair algebra with generators

- $[g]$ for any $g \in G$ on the 0-level,

- $[t]$ for any $t \in G_{\square}$ on the 1-level,

- no generators on the ee-level,

and relations

- $H[g]=0$ for $g \in G$,

- $[1]=1$ the unit element,

- $[g h]=[g] \cdot[h]$ for $g, h \in G$,

- $\partial[t]=-[\delta(t)]+\varepsilon \delta(t)$

- $[s t]=[\delta(s)] \cdot[t]+\varepsilon \delta(t) \cdot[s]+\left(\begin{array}{c}\varepsilon \delta(s) \\ 2\end{array}\right)\left(\begin{array}{c}\varepsilon \delta(t) \\ 2\end{array}\right) P(1 \mid 1)_{H}$ for $s, t \in G_{\square}$,

- $[\omega]=P(1 \mid 1)_{H}$ where $\omega=\imath(-1)$.

The relations above show that $A_{(0)}\left(G_{\square}\right)=\mathbb{Z}_{n i l}\left[G_{+}\right]$where $G_{+}$is the group $G$ together with an outer base point, so $A\left(G_{\square}\right)$ is 0 -free and hence 0 -good.

If $C$ is a 0 -good quadratic pair module then an action of $G_{\square}$ on $C$ is the same as a right ungraded $A\left(G_{\square}\right)$-module structure on $C$. The correspondence is given by the following equations, see [BM07]. Given $g \in G, t \in G_{\square}, x \in C_{0}, y \in C_{1}$, $z \in C_{e e}$,

$$
\begin{aligned}
g^{*} x & =x \cdot[g] \\
g^{*} y & =y \cdot[g], \\
g^{*} z & =z \cdot \Delta[g] \\
\langle x, t\rangle & =x \cdot[t]+\left(\begin{array}{c}
\varepsilon \delta(t) \\
2
\end{array}\right) \operatorname{PH}(x) .
\end{aligned}
$$

Definition 8.3. A morphism $f_{\square}: G_{\square} \rightarrow K_{\square}$ in the category $\mathbf{G r}_{ \pm}$of sign groups is given by two group homomorphisms $f: G \rightarrow K, f_{\square}: G_{\square} \rightarrow K_{\square}$ with $f_{\square} \imath=\imath$, $f \delta=\delta f_{\square}$, and $\varepsilon=\varepsilon f$. In this situation we say that the homomorphism $f_{\square}$ covers $f$. A twisted bilinear morphism of sign groups

$$
\left(f_{\square}, g_{\square}\right): G_{\square} \times L_{\square} \longrightarrow K_{\square}
$$

is given by a pair of sign group morphisms $f_{\square}: G_{\square} \rightarrow K_{\square}, g_{\square}: L_{\square} \rightarrow K_{\square}$, such that given $a \in G$ and $b \in L$ the equality

$$
f(a) g(b)=g(b) f(a)
$$

holds in $K$, and therefore the group homomorphism

$$
(f, g): G \times L \longrightarrow K:(a, b) \mapsto f(a) g(b)
$$

is defined, and given $x \in G_{\square}, y \in L_{\square}$ the following equality is satisfied in $K_{\square}$

$$
f_{\square}(x) g_{\square}(y)=g_{\square}(y) f_{\square}(x) \imath\left((-1)^{\left(\begin{array}{c}
\varepsilon \delta(x) \\
2
\end{array}\right)\left(\begin{array}{c}
\varepsilon \delta(y) \\
2
\end{array}\right)}\right) .
$$


There is a universal twisted bilinear morphism

$$
\left(i_{G_{\square}}, i_{L_{\square}}\right): G_{\square} \times L_{\square} \longrightarrow G_{\square} \tilde{\times} L_{\square}
$$

Here $G_{\square} \tilde{\times} L_{\square}$ is the twisted product of sign groups defined in BM07, which is a sign group

$$
\{ \pm 1\} \stackrel{\imath}{\hookrightarrow} G_{\square} \tilde{\times} L_{\square} \rightarrow G \times L \stackrel{\varepsilon}{\rightarrow}\{ \pm 1\} .
$$

The twisted product defines a non-symmetric monoidal structure in $\mathbf{G r}_{ \pm}$. We show in [BM07] that the "group ring" $A\left(G_{\square}\right)$ is a strict monoidal functor, i.e. the "group ring" of a twisted product of sign groups is the tensor product of the "group rings" of the factors and the "group ring" of the trivial sign group is $\overline{\mathbb{Z}}_{n i l}$.

Remark 8.4. The isomorphism class of a sign group $G_{\square}$ is determined by two group cohomology classes

$$
\varepsilon_{G_{\square}} \in H^{1}(G, \mathbb{Z} / 2), \quad\left\{G_{\square}\right\} \in H^{2}(G, \mathbb{Z} / 2),
$$

corresponding to the homomorphism $\varepsilon$ and the extension in Definition 8.1, respectively. For the symmetric track groups $\operatorname{Sym}_{\square}(n)$ these cohomology classes are the first two Stiefel-Whitney classes associated to the usual inclusion of the symmetric group into the orthogonal group $\operatorname{Sym}(n) \subset O(n)$. The cohomological invariants of $G_{\square} \tilde{\times} L_{\square}$ are given by

$$
\begin{aligned}
\varepsilon_{G_{\square} \tilde{\times} L_{\square}} & =p_{1}^{*} \varepsilon_{G_{\square}}+p_{2}^{*} \varepsilon_{L_{\square}} \in H^{1}(G \times L, \mathbb{Z} / 2), \\
\left\{G_{\square} \tilde{\times} L_{\square}\right\} & =p_{1}^{*}\left\{G_{\square}\right\}+p_{1}^{*} \varepsilon_{G_{\square}} \smile p_{2}^{*} \varepsilon_{L_{\square}}+p_{2}^{*}\left\{L_{\square}\right\} \in H^{2}(G \times L, \mathbb{Z} / 2) .
\end{aligned}
$$

Here $p_{1}$ and $p_{2}$ are the projections of the factors of the product $G \times K$ and $\smile$ denotes the cup-product.

We define in BM07 sign group morphisms

$$
\times_{\square}: \operatorname{Sym}_{\square}(n) \tilde{x} \operatorname{Sym}_{\square}(m) \longrightarrow \operatorname{Sym}_{\square}(n+m)
$$

covering the usual cross product homomorphisms

$$
\operatorname{Sym}(n) \times \operatorname{Sym}(m) \longrightarrow \operatorname{Sym}(n+m):(\sigma, \tau) \mapsto \sigma \times \tau .
$$

Recall that the block permutation $\sigma \times \tau$ permutes the first $n$ elements $\{1, \ldots, n\}$ of $\{1, \ldots, n, n+1, \ldots, n+m\}$ according to $\sigma$ and the last $m$ elements of this set $\{n+1, \ldots, n+m\}$ according to $\tau$. The sign group morphism (8.5) corresponds to the twisted bilinear morphism

$$
\left(-\wedge S^{m}, S^{n} \wedge-\right): \operatorname{Sym}_{\square}(n) \times \operatorname{Sym}_{\square}(m) \longrightarrow \operatorname{Sym}_{\square}(n+m)
$$

defined on generators by

$$
\begin{aligned}
t_{i} \wedge S^{m} & =t_{i}, \quad 1 \leq i \leq n, \\
\omega \wedge S^{m} & =\omega, \\
S^{n} \wedge t_{i} & =t_{n+i}, \quad 1 \leq i \leq m, \\
S^{n} \wedge \omega & =\omega .
\end{aligned}
$$

The sign group morphism (8.5) was defined in BM07] in a geometric way. We obtained this description in terms of generators by using the positive pin representations in [BM06c and the computations in [BM07, Section 17], see [BM06b for further details. 


\section{9. $E_{\infty}$-QUADRATIC PAIR ALGEBRAS ASSOCIATED TO COMMUTATIVE RING SPECTRA}

In order to deal with secondary operations in the homotopy groups of a connective commutative ring spectrum in general we need the following algebraic structure.

Definition 9.1. An $E_{\infty}$-quadratic pair algebra is a quadratic pair algebra $C$ as in Definition 6.1 together with a cup-one product operation

$$
\smile_{1}: C_{n, 0} \times C_{m, 0} \longrightarrow C_{n+m, 1}, \quad n, m \geq 0,
$$

such that the quadratic pair module $C_{n, *}$ is endowed with a right action of the symmetric track group $\operatorname{Sym}_{\square}(n)$ and the following compatibility conditions hold. Let $x_{i} \in C_{n_{i}, 0}, s_{i} \in C_{n_{i}, 1}, a_{i} \in C_{n_{i}, e e}, g_{i}, g_{i}^{\prime} \in \operatorname{Sym}\left(n_{i}\right)$, and $r_{i} \in \operatorname{Sym}_{\square}\left(n_{i}\right)$. The product in the quadratic pair algebra $C$ is compatible with the sign group actions and the sign group morphisms $\times_{\square}$ in (8.5),

$$
\begin{aligned}
\left(x_{1} \cdot\left[g_{1}\right]\right) \cdot\left(x_{2} \cdot\left[g_{2}\right]\right) & =\left(x_{1} \cdot x_{2}\right) \cdot\left[g_{1} \times g_{2}\right], \\
\left(s_{1} \cdot\left[g_{1}\right]\right) \cdot\left(x_{2} \cdot\left[g_{2}\right]\right) & =\left(s_{1} \cdot x_{2}\right) \cdot\left[g_{1} \times g_{2}\right], \\
\left(a_{1} \cdot\left(\left[g_{1}\right] \mid\left[g_{1}^{\prime}\right]\right)_{H}\right) \cdot\left(a_{2} \cdot\left(\left[g_{2}\right] \mid\left[g_{2}^{\prime}\right]\right)_{H}\right) & =\left(a_{1} \cdot a_{2}\right) \cdot\left(\left[g_{1} \times g_{2}\right] \mid\left[g_{1}^{\prime} \times g_{2}^{\prime}\right]\right)_{H}, \\
x_{1} \cdot\left(x_{2} \cdot\left[r_{2}\right]\right) & =\left(x_{1} \cdot x_{2}\right) \cdot\left[S^{n_{1}} \wedge r_{2}\right] .
\end{aligned}
$$

The cup-one product measures the lack of commutativity, i.e. if $\tau_{p, q} \in \operatorname{Sym}(p+q)$ denotes the permutation exchanging the blocks $\{1, \ldots, p\}$ and $\{p+1, \ldots, p+q\}$, $p, q \geq 0$, then

$$
\begin{aligned}
&\left(x_{2} \cdot x_{1}\right) \cdot\left[\tau_{n_{1}, n_{2}}\right]+\partial\left(x_{1} \smile_{1} x_{2}\right)=x_{1} \cdot x_{2}+\partial P\left(H\left(x_{2}\right) \cdot T H\left(x_{1}\right)\right) \cdot\left[\tau_{n_{1}, n_{2}}\right], \\
&\left(x_{2} \cdot s_{1}\right) \cdot\left[\tau_{n_{1}, n_{2}}\right]+\partial\left(s_{1}\right) \smile_{1} x_{2}=s_{1} \cdot x_{2}+P\left(H\left(x_{2}\right) \cdot T H \partial\left(s_{1}\right)\right) \cdot\left[\tau_{n_{1}, n_{2}}\right] .
\end{aligned}
$$

The cup-one product is itself commutative in the following sense

$$
\begin{aligned}
\left(x_{2} \smile_{1} x_{1}\right) \cdot\left[\tau_{n_{1}, n_{2}}\right]+x_{1} \smile_{1} x_{2}= & -P\left(T H\left(x_{1}\right) \cdot H\left(x_{2}\right)\right) \\
& +P\left(H\left(x_{2}\right) \cdot T H\left(x_{1}\right)\right) \cdot\left[\tau_{n_{1}, n_{2}}\right] .
\end{aligned}
$$

Let $1_{n} \in \operatorname{Sym}(n)$ be the unit element. The cup-one product also satisfies the following rules with respect to addition

$$
\begin{aligned}
x_{1} \smile_{1}\left(x_{2}+x_{3}\right)= & x_{1} \smile_{1} x_{2}+x_{1} \smile_{1} x_{3} \\
& +P\left(\partial\left(x_{1} \smile_{1} x_{2}\right) \mid\left(x_{3} \cdot x_{1}\right) \cdot\left[\tau_{n_{1}, n_{3}}\right]\right)_{H},
\end{aligned}
$$

multiplication

$$
\begin{aligned}
\left(x_{1} \cdot x_{2}\right) \smile_{1} x_{3}= & \left(\left(x_{1} \smile_{1} x_{3}\right) \cdot x_{2}\right) \cdot\left[1_{n_{1}} \times \tau_{n_{2}, n_{3}}\right]+x_{1} \cdot\left(x_{2} \smile_{1} x_{3}\right) \\
& +P\left(\left(\partial\left(x_{1} \smile_{1} x_{3}\right) \mid\left(x_{3} \cdot x_{1}\right) \cdot\left[\tau_{n_{1}, n_{3}}\right]\right)_{H} \cdot H\left(x_{2}\right)\right) \cdot\left[1_{n_{1}} \times \tau_{n_{2}, n_{3}}\right] \\
& +P\left(H\left(x_{3}\right) \cdot\left(x_{1} \mid x_{1}\right)_{H} \cdot T H\left(x_{2}\right)\right) \cdot\left[\tau_{n_{1}+n_{2}, n_{3}}\right] \\
& -P\left(\left(x_{1} \mid x_{1}\right)_{H} \cdot H\left(x_{3}\right) \cdot T H\left(x_{2}\right)\right) \cdot\left[1_{n_{1}} \times \tau_{n_{2}, n_{3}}\right],
\end{aligned}
$$

and symmetric group action

$$
\left(x_{1} \cdot\left[g_{1}\right]\right) \smile_{1}\left(x_{2} \cdot\left[g_{2}\right]\right)=\left(x_{1} \smile_{1} x_{2}\right) \cdot\left[g_{1} \times g_{2}\right] .
$$

Remark 9.8. One can check that an $E_{\infty}$-pair algebra $C$ as defined in Definition 3.1 is the same as an $E_{\infty}$-quadratic pair algebra such that $C_{*, e e}=0$ and for any $x \in C_{n, 0}$ and $t \in \operatorname{Sym}_{\square}(n)$ the equation $x \cdot[t]=0$ holds. This defines a full inclusion of categories

$$
\left(E_{\infty} \text {-pair algebras }\right) \subset\left(E_{\infty} \text {-quadratic pair algebras }\right) .
$$


Lemma 9.9. Given an $E_{\infty}$-quadratic pair algebra $C$ the ring $h_{0} C$ is commutative and $h_{1} C$ is an $h_{0} C$-module in a natural way.

Proof. Let $x_{i} \in C_{n_{i}, 0}$. By the laws of an $E_{\infty}$-quadratic pair algebra the class of $x_{1} \cdot x_{2}$ in $h_{0} C$ coincides with the class of $\left(x_{2} \cdot x_{1}\right) \cdot\left[\tau_{n_{1}, n_{2}}\right]$. It is easy to see that $\operatorname{sign} \tau_{n_{1}, n_{2}}=(-1)^{n_{1} n_{2}}$. Let $\hat{\tau} \in \operatorname{Sym}_{\square}\left(n_{1}+n_{2}\right)$ be an element with $\delta(\hat{\tau})=\tau_{n_{1}, n_{2}}$. By the laws of a module over a quadratic pair algebra and the definition of the "group ring" of a sign group

$$
\begin{aligned}
\partial\left(\left(x_{2} \cdot x_{1}\right) \cdot[\hat{\tau}]\right) & =\left(x_{2} \cdot x_{1}\right) \cdot \partial[\hat{\tau}] \\
& =\left(x_{2} \cdot x_{1}\right) \cdot\left(-\left[\tau_{n_{1}, n_{2}}\right]+(-1)^{n_{1} n_{2}}\right) \\
& =-\left(x_{2} \cdot x_{1}\right) \cdot\left[\tau_{n_{1}, n_{2}}\right]+(-1)^{n_{1} n_{2}}\left(x_{2} \cdot x_{1}\right),
\end{aligned}
$$

therefore $\left(x_{2} \cdot x_{1}\right) \cdot\left[\tau_{n_{1}, n_{2}}\right]$ represents the same class as $(-1)^{n_{1} n_{2}}\left(x_{2} \cdot x_{1}\right)$ in $h_{0} C$, so $h_{0} C$ is commutative.

Let now $s_{1} \in C_{n_{1}, 1}$ be an element with $\partial\left(s_{1}\right)=0$. By the laws of an $E_{\infty^{-}}$quadratic pair algebra $\left(x_{2} \cdot s_{1}\right) \cdot\left[\tau_{n_{1}, n_{2}}\right]=s_{1} \cdot x_{2}$. Moreover, by the laws of quadratic pair algebras, modules over a quadratic pair algebra, and the definition of the "group ring" of a sign group

$$
\begin{aligned}
0 & =x_{2} \cdot \partial\left(s_{1}\right) \cdot[\hat{\tau}] \\
& =x_{2} \cdot s_{1} \cdot \partial[\hat{\tau}] \\
& =x_{2} \cdot s_{1} \cdot\left(-\left[\tau_{n_{1}, n_{2}}\right]+(-1)^{n_{1} n_{2}}\right) \\
& =-\left(x_{2} \cdot s_{1}\right) \cdot\left[\tau_{n_{1}, n_{2}}\right]+(-1)^{n_{1} n_{2}}\left(x_{2} \cdot s_{1}\right),
\end{aligned}
$$

hence we are done.

As in the non-quadratic case $E_{\infty}$-quadratic pair algebras carry a notion of cupone square.

Definition 9.10. Let $C$ be an $E_{\infty}$-quadratic pair algebra. Given an element $a \in h_{0} C_{2 n, *}$ we define the cup-one square of $a$

$$
S q_{1}(a) \in h_{1} C_{4 n, *}
$$

in the following way. Choose a representative $\bar{a} \in C_{2 n, 0}$ of $a$ and an element in the symmetric track group $\hat{\tau} \in \operatorname{Sym}_{\square}(4 n)$ whose boundary is the shuffle permutation $\delta(\hat{\tau})=\tau_{2 n, 2 n}$. Then we have

$$
\partial\left(-(\bar{a} \cdot \bar{a}) \cdot[\hat{\tau}]+\bar{a} \smile_{1} \bar{a}-P(H(\bar{a}) \cdot T H(\bar{a})) \cdot\left[\tau_{2 n, 2 n}\right]\right)=0 .
$$

so we can set

$$
S q_{1}(a)=-(\bar{a} \cdot \bar{a}) \cdot[\hat{\tau}]+\bar{a} \smile_{1} \bar{a}-P(H(\bar{a}) \cdot T H(\bar{a})) \cdot\left[\tau_{2 n, 2 n}\right] \in h_{1} C_{4 n, *} .
$$

We leave it to the reader to check that this element does not depend on the choice of $\bar{a}$. However it does depend on the choice of $\hat{\tau}$. There are two possible choices, namely $\hat{\tau}$ and $\omega \hat{\tau}$. The next result determines the difference between the two possible cup-one squares.

Lemma 9.11. Let $S q_{1}$ be cup-one square in an $E_{\infty}$-quadratic pair algebra $C$ associated to the lift $\hat{\tau}$ of the shuffle permutation and let $S q_{1}^{\omega}$ be the cup-one square associated to $\omega \hat{\tau}$. Then given $a \in h_{0} C_{2 n, *}$

$$
S q_{1}^{\omega}(a)=S q_{1}(a)+k(a \cdot a) .
$$

Here $k$ is the $k$-invariant defined in (5.4). 
Proof. Let $\bar{a} \in C_{2 n, 0}$ be a representative of $a$. The laws of a quadratic pair algebra, of a module over it, and the definition of the "group ring" of a sign group yield equations

$$
\begin{aligned}
(\bar{a} \cdot \bar{a}) \cdot[\omega \hat{\tau}] & =(\bar{a} \cdot \bar{a}) \cdot([\hat{\tau}]+[\omega]) \\
& =(\bar{a} \cdot \bar{a}) \cdot[\hat{\tau}]+(\bar{a} \cdot \bar{a}) \cdot[\omega] \\
& =(\bar{a} \cdot \bar{a}) \cdot[\hat{\tau}]+(\bar{a} \cdot \bar{a}) \cdot P(1 \mid 1)_{H} \\
& =(\bar{a} \cdot \bar{a}) \cdot[\hat{\tau}]+P(\bar{a} \cdot \bar{a} \mid \bar{a} \cdot \bar{a})_{H},
\end{aligned}
$$

hence the lemma follows from the very definition of the cup-one product and the $k$-invariant of a quadratic pair module.

One of the main results in this paper is the following theorem.

Theorem 9.12. There is a commutative diagram of functors

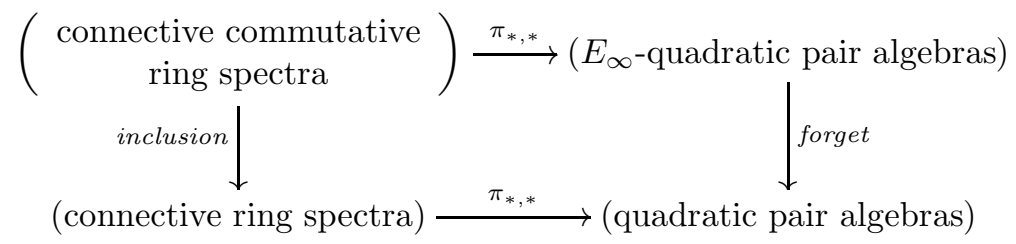

Here the lower arrow is the functor in Theorem 6.4. Moreover, for a commutative ring spectrum $Q$ the algebraic cup-one squares in $\pi_{*, *} Q$ correspond to the topologically-defined cup-one squares in $\pi_{*} Q$.

We prove this theorem in Section 16.

Remark 9.13. $E_{\infty}$-quadratic pair algebras can also be described as coherently homotopy commutative monoids as in the additive case, see Remark 3.2. For this one uses the symmetric monoidal category $\mathbf{q p m}_{H}^{\mathrm{Sym}} \square$ of enhanced symmetric sequences of 0-good quadratic pair modules introduced in BM07]. In fact, this is the way in which we obtain the equations in Definition 9.1. The cup-one product $x_{1} \smile_{1} x_{2}$ of two level 0 elements corresponds to the image of the right linear generator $x_{1} \odot x_{2}$ in the tensor product of square groups $C_{*,(0)} \odot C_{*,(0)}=(C \odot C)_{*,(0)}$ under the track $\smile_{1}$. The definition of tracks for quadratic pair modules is recalled in Definition 11.4 below.

As we recall in Remark 3.8 the coherence conditions in Remark 3.2 (i.e. the hexagon and the idempotence) are encoded by the Barratt-Eccles operad in the simplicial setting. Indeed, $E_{\infty}$-quadratic pair algebras are also the algebras over an operad in $\mathbf{q p m}_{H}$ which can be obtained from the simplicial Barratt-Eccles operad, i.e. the additive situation described in Remark 3.8 extends to the quadratic world.

Remark 9.14. If $C$ is an ungraded $E_{\infty}$-quadratic pair algebra then the permutative category $\mathbf{P C}$ in Remark 5.9 is actually a bipermutative category in the sense of EM05, 3.6]. The monoidal structures $\oplus$ and $\otimes$ are given by Remarks 5.9 and 6.9. and the symmetry isomorphism for $\otimes$ is

$$
\left(x_{1} \cdot x_{2}, P\left(H\left(x_{2}\right) \cdot T H\left(x_{1}\right)\right)-x_{1} \smile_{1} x_{2}\right): x_{1} \otimes x_{2} \longrightarrow x_{2} \otimes x_{1} .
$$

\section{Algebras over Commutative Ring SPECtra}

Given a connective commutative ring spectrum $Q$ and a $Q$-algebra $R$ we study in this section the algebraic properties of the induced action of the $E_{\infty}$-quadratic pair algebra $\pi_{*, *} Q$ defined by Theorem 9.12 on the quadratic pair algebra $\pi_{*, *} R$ given by Theorem 6.4 obtaining Theorem [1.5 as an application. This generalizes the results of Section 4 to spectra which do not neglect the Hopf map. 
Definition 10.1. Let $C$ be an $E_{\infty}$-quadratic pair algebra. A $C$-algebra is a quadratic pair algebra $B$ as in Definition 6.1 together with a quadratic pair algebra morphism $u: C \rightarrow B$, called the unit, and two external cup-one product operations, $n, m \geq 0$,

$$
\begin{aligned}
& \smile_{1}: B_{n, 0} \times C_{m, 0} \longrightarrow C_{n+m, 1}, \\
& \smile_{1}: C_{n, 0} \times B_{m, 0} \longrightarrow C_{n+m, 1},
\end{aligned}
$$

such that the quadratic pair module $B_{n, *}$ is endowed with a right action of the symmetric track group $\operatorname{Sym}_{\square}(n)$ and the following compatibility conditions hold. Let $x_{i} \in B_{n_{i}, 0}, s_{i} \in B_{n_{i}, 1}, a_{i} \in B_{n_{i}, e e}, \tilde{x}_{i} \in C_{n_{i}, 0}, \tilde{s}_{i} \in C_{n_{i}, 1}, \tilde{a}_{i} \in C_{n_{i}, e e}$, $g_{i}, g_{i}^{\prime} \in \operatorname{Sym}\left(n_{i}\right)$, and $r_{i} \in \operatorname{Sym}_{\square}\left(n_{i}\right)$. The product in $B$ is equivariant, i.e. it satisfies equations (9.2) and

$$
\left(x_{1} \cdot\left[r_{1}\right]\right) \cdot x_{2}=\left(x_{1} \cdot x_{2}\right) \cdot\left[r_{1} \wedge S^{n_{2}}\right] .
$$

The morphism $u$ is also equivariant,

$$
\begin{aligned}
& u_{0}\left(\tilde{x}_{1} \cdot\left[g_{1}\right]\right)=u_{0}\left(\tilde{x}_{1}\right) \cdot\left[g_{1}\right], \\
& u_{1}\left(\tilde{s}_{1} \cdot\left[g_{1}\right]\right)=u_{1}\left(\tilde{s}_{1}\right) \cdot\left[g_{1}\right], \\
& u_{1}\left(\tilde{x}_{1} \cdot\left[r_{1}\right]\right)=u_{0}\left(\tilde{x}_{1}\right) \cdot\left[r_{1}\right],
\end{aligned}
$$

and compatible with the cup-one products in $C$ and $B$

$$
\begin{aligned}
u_{1}\left(\tilde{x}_{1} \smile_{1} \tilde{x}_{2}\right) & =u_{0}\left(\tilde{x}_{1}\right) \smile_{1} \tilde{x}_{2}, \\
& =\tilde{x}_{1} \smile_{1} u_{0}\left(\tilde{x}_{2}\right) .
\end{aligned}
$$

The external cup-one products satisfy the following analogue of (9.3),

$$
\begin{aligned}
\left(x_{2} \cdot u_{0}\left(\tilde{x}_{1}\right)\right) \cdot\left[\tau_{n_{1}, n_{2}}\right]+\partial\left(\tilde{x}_{1} \smile_{1} x_{2}\right) & =u_{0}\left(\tilde{x}_{1}\right) \cdot x_{2}+\partial P\left(H\left(x_{2}\right) \cdot T H u_{0}\left(\tilde{x}_{1}\right)\right) \cdot\left[\tau_{n_{1}, n_{2}}\right], \\
\left(x_{2} \cdot u_{1}\left(\tilde{s}_{1}\right)\right) \cdot\left[\tau_{n_{1}, n_{2}}\right]+\partial\left(\tilde{s}_{1}\right) \smile_{1} x_{2}=u_{1}\left(\tilde{s}_{1}\right) \cdot x_{2}+P\left(H\left(x_{2}\right) \cdot T H \partial u_{1}\left(\tilde{s}_{1}\right)\right) \cdot\left[\tau_{n_{1}, n_{2}}\right], & \left(\tilde{x}_{1}\right) \smile_{1} \tilde{x}_{2}=s_{1} \cdot u_{0}\left(\tilde{x}_{2}\right)+P\left(H u_{0}\left(\tilde{x}_{2}\right) \cdot T H \partial\left(s_{1}\right)\right) \cdot\left[\tau_{n_{1}, n_{2}}\right] .
\end{aligned}
$$

The two external cup-one products are related by the following formula, which is similar to (9.4),

$$
\begin{aligned}
\left(\tilde{x}_{2} \smile_{1} x_{1}\right) \cdot\left[\tau_{n_{1}, n_{2}}\right]+x_{1} \smile_{1} \tilde{x}_{2}= & -P\left(T H\left(x_{1}\right) \cdot H u_{0}\left(\tilde{x}_{2}\right)\right) \\
& +P\left(H u_{0}\left(\tilde{x}_{2}\right) \cdot T H\left(x_{1}\right)\right) \cdot\left[\tau_{n_{1}, n_{2}}\right] .
\end{aligned}
$$

in particular it is redundant to give the two external cup-one products in (10.2) since any of them determines the other, but having two makes the equations shorter. The external cup-one products also satisfy the following rules with respect to addition

$x_{1} \smile_{1}\left(\tilde{x}_{2}+\tilde{x}_{3}\right)=x_{1} \smile_{1} \tilde{x}_{2}+x_{1} \smile_{1} \tilde{x}_{3}+P\left(\partial\left(x_{1} \smile_{1} \tilde{x}_{2}\right) \mid\left(u_{0}\left(\tilde{x}_{3}\right) \cdot x_{1}\right) \cdot\left[\tau_{n_{1}, n_{3}}\right]\right)_{H}$, $\tilde{x}_{1} \smile_{1}\left(x_{2}+x_{3}\right)=\tilde{x}_{1} \smile_{1} x_{2}+\tilde{x}_{1} \smile_{1} x_{3}+P\left(\partial\left(\tilde{x}_{1} \smile_{1} x_{2}\right) \mid\left(x_{3} \cdot u_{0}\left(\tilde{x}_{1}\right)\right) \cdot\left[\tau_{n_{1}, n_{3}}\right]\right)_{H}$,

compare (9.5), multiplication

$$
\begin{aligned}
\left(\tilde{x}_{1} \cdot \tilde{x}_{2}\right) \smile_{1} x_{3}= & \left(\left(\tilde{x}_{1} \smile_{1} x_{3}\right) \cdot u_{0}\left(\tilde{x}_{2}\right)\right) \cdot\left[1_{n_{1}} \times \tau_{n_{2}, n_{3}}\right]+u_{0}\left(\tilde{x}_{1}\right) \cdot\left(\tilde{x}_{2} \smile_{1} x_{3}\right) \\
& +P\left(\left(\partial\left(\tilde{x}_{1} \smile_{1} x_{3}\right) \mid\left(x_{3} \cdot u_{0}\left(\tilde{x}_{1}\right)\right) \cdot\left[\tau_{n_{1}, n_{3}}\right]\right)_{H} \cdot H u_{0}\left(\tilde{x}_{2}\right)\right) \cdot\left[1_{n_{1}} \times \tau_{n_{2}, n_{3}}\right] \\
& +P\left(H\left(x_{3}\right) \cdot u_{e e}\left(\tilde{x}_{1} \mid \tilde{x}_{1}\right)_{H} \cdot T H u_{0}\left(\tilde{x}_{2}\right)\right) \cdot\left[\tau_{n_{1}+n_{2}, n_{3}}\right] \\
& -P\left(u_{e e}\left(\tilde{x}_{1} \mid \tilde{x}_{1}\right)_{H} \cdot H\left(x_{3}\right) \cdot T H u_{0}\left(\tilde{x}_{2}\right)\right) \cdot\left[1_{n_{1}} \times \tau_{n_{2}, n_{3}}\right], \\
\left(x_{1} \cdot x_{2}\right) \smile_{1} \tilde{x}_{3}= & \left(\left(x_{1} \smile_{1} \tilde{x}_{3}\right) \cdot x_{2}\right) \cdot\left[1_{n_{1}} \times \tau_{n_{2}, n_{3}}\right]+x_{1} \cdot\left(x_{2} \smile_{1} \tilde{x}_{3}\right) \\
& +P\left(\left(\partial\left(x_{1} \smile_{1} \tilde{x}_{3}\right) \mid\left(u_{0}\left(\tilde{x}_{3}\right) \cdot x_{1}\right) \cdot\left[\tau_{n_{1}, n_{3}}\right]\right)_{H} \cdot H\left(x_{2}\right)\right) \cdot\left[1_{n_{1}} \times \tau_{n_{2}, n_{3}}\right] \\
& +P\left(H u_{0}\left(\tilde{x}_{3}\right) \cdot\left(x_{1} \mid x_{1}\right)_{H} \cdot T H\left(x_{2}\right)\right) \cdot\left[\tau_{n_{1}+n_{2}, n_{3}}\right] \\
& -P\left(\left(x_{1} \mid x_{1}\right)_{H} \cdot H u_{0}\left(\tilde{x}_{3}\right) \cdot T H\left(x_{2}\right)\right) \cdot\left[1_{n_{1}} \times \tau_{n_{2}, n_{3}}\right],
\end{aligned}
$$


compare (9.6), and symmetric group action

$$
\begin{aligned}
& \left(x_{1} \cdot\left[g_{1}\right]\right) \smile_{1}\left(\tilde{x}_{2} \cdot\left[g_{2}\right]\right)=\left(x_{1} \smile_{1} \tilde{x}_{2}\right) \cdot\left[g_{1} \times g_{2}\right], \\
& \left(\tilde{x}_{1} \cdot\left[g_{1}\right]\right) \smile_{1}\left(x_{2} \cdot\left[g_{2}\right]\right)=\left(\tilde{x}_{1} \smile_{1} x_{2}\right) \cdot\left[g_{1} \times g_{2}\right],
\end{aligned}
$$

compare (9.7).

The ring $h_{0} B$ is an $h_{0} C$-algebra in such a way that the $h_{0} B$-bimodule $h_{1} B$ restricts to an $h_{0} C$-module.

Remark 10.3. Any $E_{\infty}$-quadratic pair algebra is an algebra over itself. Moreover, given a morphism of $E_{\infty}$-quadratic pair algebras $f: \bar{C} \rightarrow C$ and a $C$-algebra $B$ then $B$ has a $\bar{C}$-algebra structure, denoted by $f^{*} B$, with unit $u f: \bar{C} \rightarrow B$ and external cup-one products obtained by precomposing with $f$.

The main new secondary homotopy operation on algebras over $E_{\infty}$-quadratic pair algebras is introduced in the next definition, analogous to Definition 4.10.

Definition 10.4. Let $C$ be an $E_{\infty}$-quadratic pair algebra and let $B$ be a $C$-algebra. We associate to any pair $(x, y)$ with $x \in C_{0, n}$ and $y \in B_{n, 1}$ such that $\partial(y)=u_{0}(x)$ the degree $n$ derivation

$$
\theta_{(x, y)}: h_{0} B \longrightarrow h_{1} B
$$

defined by

$$
\theta_{(x, y)}(a)=-y \cdot \bar{a}+(\bar{a} \cdot y) \cdot\left[\tau_{n,|a|}\right]+x \smile_{1} \bar{a}-P\left(H(\bar{a}) \cdot T H u_{0}(x)\right) .
$$

Here $\bar{a} \in B_{*, 0}$ is a representative of $a \in h_{0} B$. This is indeed an element in $h_{1} B$ by the equations defining a $C$-algebra which are analogue to (9.3), see Definition 10.1. Moreover, by (9.3) $\theta_{(x, y)}=0$ provided $B$ is an $E_{\infty}$-quadratic pair algebra and $u$ is a morphism of $E_{\infty}$-quadratic pair algebras. One readily checks that $\theta_{(x, y)}$ is indeed a derivation and that $\theta_{(x, y)}(a)$ does not depend on the choice of $\bar{a}$.

Proposition 10.5. Given an $E_{\infty}$-quadratic pair algebra $C$ and a $C$-algebra $B$ let $I_{C}(B)$ be the kernel of the ring homomorphism $h_{0} u: h_{0} C \rightarrow h_{0} B$. There is an $h_{0} C$-module homomorphism

$$
\theta: I_{C}(B) / I_{C}(B)^{2} \longrightarrow H H_{h_{0} C}^{1}\left(h_{0} B, h_{1} B\right)
$$

which sends an element in $I_{C}(B) / I_{C}(B)^{2}$ represented by $x \in C_{n, 0}$ to the element in Hochschild cohomology represented by a derivation $\theta_{(x, y)}$ as in Definition 10.4 for any $y \in B_{n, 1}$ with $\partial(y)=u_{0}(x)$. The homomorphism $\theta$ is natural in $B$ and in $C$ in the obvious way. In particular $\theta=0$ when $B$ is an $E_{\infty}$-quadratic pair algebra and $u$ is a morphism of $E_{\infty}$-quadratic pair algebras.

The proof of this proposition is tedious but straightforward. It only uses the equations defining algebras over $E_{\infty}$-quadratic pair algebras, compare the proof of the additive analogue, Proposition 4.11.

Algebras over commutative ring spectra yield examples of algebras over $E_{\infty^{-}}$ quadratic pair algebras as the following theorem shows.

Theorem 10.6. Let $Q$ be a connective commutative ring spectrum and let $\pi_{*, *} Q$ be the $E_{\infty}$-quadratic pair algebra given by Theorem 9.12. There is a diagram of functors

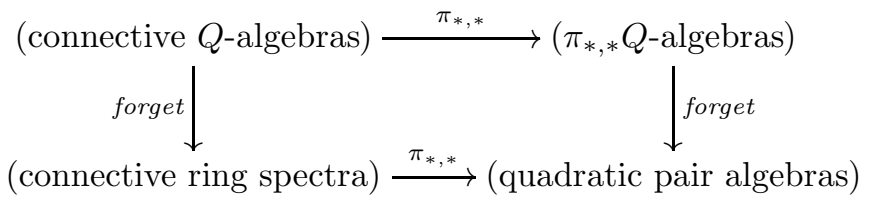


which commutes up to quasi-isomorphisms. Here the lower arrow is the functor in Theorem 6.4. Moreover, if $f: Q^{\prime} \rightarrow Q$ is a morphism of connective commutative ring spectra then the square

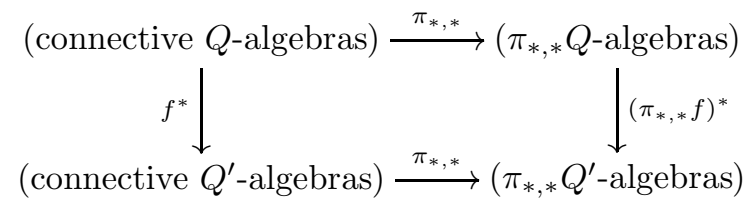

commutes up to quasi-isomorphisms. Furthermore, if $R$ is a connective $Q$-algebra, $a \in \pi_{m} R$, and $b \in \pi_{n} Q$ is in $I_{Q}(R)=\operatorname{Ker}\left[\pi_{*} Q \rightarrow \pi_{*} R\right] \cong I_{\pi_{* * *} Q}\left(\pi_{*, *} R\right)$, then the element $\theta_{(x, y)}(a) \in \pi_{n+m+1} R \cong\left(h_{1} \pi_{*, *} R\right)_{n+m}$ defined in (1.4) can be identified with $\theta_{(x, y)}(a)$ in the sense of Definition 10.4 .

The proof of this theorem goes along the same lines as the proof of Theorem 9.12. The reader can find the crucial hints in the following remark.

Remark 10.7. Suppose that $Q$ is a connective commutative ring spectrum, $R$ is a connective $Q$-algebra, and $\bar{L}$ is a functorial fibrant replacement in the category of $Q$-algebras. We assume without loss of generality that $\bar{L} Q$ concides with the fibrant relplacement chosen for the definition of $\pi_{*, *} Q$ in the proof of Theorem 9.12 (otherwise we would have to work with a quasi-isomorphic $E_{\infty}$-quadratic pair algebra). Then $\pi_{*, *} R$ is defined by the secondary homotopy groups of the spectrum $\bar{L} R$, as defined in Section 15. The unit $\bar{u}: Q \rightarrow R$ of the $Q$-algebra $R$ and the product in $\bar{L} R$ induce maps

$$
\begin{aligned}
& \mu_{1}: \bar{L} Q \wedge_{Q} \bar{L} R \stackrel{\bar{L} \bar{u} \wedge_{Q} 1}{\longrightarrow} \bar{L} R \wedge_{Q} \bar{L} R \stackrel{\text { mult. }}{\longrightarrow} \bar{L} R, \\
& \mu_{2}: \bar{L} R \wedge_{Q} \bar{L} Q \stackrel{\wedge_{Q} \bar{L} \bar{u}}{\longrightarrow} \bar{L} R \wedge_{Q} \bar{L} R \stackrel{\text { mult. }}{\longrightarrow} \bar{L} R,
\end{aligned}
$$

such that there is a track $\alpha_{1}: \mu_{1} \tau_{\wedge_{Q}} \Rightarrow \mu_{2}$ which can be constructed as in Lemma 16.2 below. The external cup-one product

$$
\smile_{1}: \pi_{*, 0} R \times \pi_{*, 0} Q \longrightarrow \pi_{*, 1} R
$$

corresponds to the track $\alpha_{1}$, and the other external cup-one product corresponds to $\alpha_{1} \tau_{\wedge_{Q}}$, compare the proof of Theorem 9.12

If $L$ is the fibrant replacement functor in the category of ring spectra used to define $\pi_{*, *}$ in Theorems 6.4 and 9.12 then the natural quasi-isomorphisms making commutative the first square in Theorem 10.6 are obtained by taking secondary homotopy groups on

$$
\bar{L} R \longmapsto L \bar{L} R \leftarrow L R .
$$

For the second square one can argue in a similar way.

With the notation used for the definition of $\theta_{(x, y)}(a)$ in (1.4), the elements $x$ and $y$ lie in $\pi_{n, 0} Q$ and $\pi_{n, 1} R$, respectively, and satisfy $\partial(y)=u_{0}(x)$ as required in Definition 10.4, therefore the last part of the statement of Theorem 10.6 makes sense.

\section{Universal TODA BRACKETS OF RING SPECTRA}

Given a ring spectrum $R$ the category $\bmod (R)$ of right $R$-modules is a Quillen model category, see [MMSS01, Theorem 12.1]. By [Bau89] the full subcategory $\bmod ^{\mathrm{fc}}(R)$ of fibrant-cofibrant right $R$-modules is a groupoid-enriched category whose morphisms are homotopy classes of homotopies, also termed tracks.

A free right $R$-module is a right $R$-module of the form

$$
R^{\left(n_{1}, \ldots, n_{k}\right)}=\left(S^{n_{1}} \vee \cdots \vee S^{n_{k}}\right) \wedge R, \quad k \geq 0, n_{1}, \ldots, n_{k} \geq 0 .
$$


Here $\vee$ denotes the coproduct. Free right $R$-modules are cofibrant objects in $\bmod (R)$ by $\left[\right.$ MMSS01, Theorem 12.1 (vi)] since sphere spectra $S^{m}$ are cofibrant in the category of symmetric spectra.

We denote by $\bmod _{f}(R)$ the full groupoid-enriched subcategory of $\bmod ^{\mathrm{fc}}(R)$ consisting of fibrant replacements of free right $R$-modules. An object $X$ in $\bmod _{f}(R)$ is a trivial cofibration $R^{\left(n_{1}, \ldots, n_{k}\right)} \stackrel{\sim}{\longmapsto} X$ in $\bmod (R)$ with fibrant target. This groupoidenriched category has a further structure described in the following definition.

Definition 11.1. Let $\mathbf{A}$ be a category and $D: \mathbf{A}^{o p} \times \mathbf{A} \rightarrow \mathbf{A b}$ a functor, also called A-bimodule. A linear track extension of $\mathbf{A}$ by $D$ consists of

(1) a groupoid-enriched category $\mathbf{B}$.

(2) A functor $p: \mathbf{B} \rightarrow \mathbf{A}$ from the underlying ordinary category of $\mathbf{B}$ such that $p$ is the identity on objects and $p(f)=p(g)$ if and only if there exists a track $f \Rightarrow g$, i.e. A is the homotopy category of $\mathbf{B}$.

(3) Isomorphisms

$$
\sigma_{f}: D(X, Y) \cong \operatorname{Aut}_{\mathbf{B}}(f)
$$

for all morphisms $f: X \rightarrow Y$ in $\mathbf{B}$ such that given a track $\alpha: f \Rightarrow g$ in $\mathbf{B}$,

$$
\alpha \square \sigma_{f}(x)=\sigma_{g}(x) \square \alpha ;
$$

and given composable morphisms $\bullet \stackrel{h}{\rightarrow} \bullet \stackrel{g}{\rightarrow} \bullet \stackrel{f}{\rightarrow} \bullet$ in $\mathbf{B}$

$$
f \sigma_{g}(x)=\sigma_{f g}(D(1, p(f)) x) \text { and } \sigma_{g}(x) h=\sigma_{g h}(D(p(h), 1) x) .
$$

For any category $\mathbf{A}$ and any functor $D: \mathbf{A}^{o p} \times \mathbf{A} \rightarrow \mathbf{A b}$ the third BauesWirsching cohomology group $H^{3}(\mathbf{A}, D)$ (which in this case is isomorphic to a Hochschild-Mitchell cohomology) classifies linear track extensions of $\mathbf{A}$ by $D$, see BD89. Indeed any such linear track extension B determines a characterisic cohomology class

$$
\langle\mathbf{B}\rangle \in H^{3}(\mathbf{A}, D)
$$

also termed universal Toda bracket, and all cohomology classes are represented by linear track extensions.

Given an $\mathbb{N}$-graded ring $A$ a free right $A$-module is a right $A$-module of the form

$$
A^{\left(n_{1}, \ldots, n_{k}\right)}=\left(\left(\mathbb{Z}, n_{1}\right) \oplus \cdots \oplus\left(\mathbb{Z}, n_{k}\right)\right) \otimes A, \quad k \geq 0, n_{1}, \ldots, n_{k} \geq 0 .
$$

Here $(\mathbb{Z}, m)$ denotes the $\mathbb{N}$-graded abelian group consisting of $\mathbb{Z}$ concentrated in degree $m$. The category of free right $A$-modules is denoted by $\bmod _{f}(A)$.

Given an $A$-bimodule $M$ we define the graded version of Mac Lane cohomology by

$$
H M L^{*}(A, M)=H^{*}\left(\bmod _{f}(A), \operatorname{Hom}_{A}\left(-,-\otimes_{A} M\right)\right) .
$$

For an ungraded ring $A$ if $\bmod _{f}(A)$ denotes the usual category of finitely generated free ungraded right $A$-modules this equality corresponds to the natural isomorphism established in [PW92] in homology.

Lemma 11.2. Let $R$ be a connective spectrum. The category $\bmod _{f}(R)$ is a linear track extension of $\bmod _{f}\left(\pi_{*} R\right)$ by $\operatorname{Hom}_{\pi_{*} R}\left(-,-\otimes_{\pi_{*} R} \Sigma^{-1} \pi_{*} R\right)$.

Proof. For any right $R$-module $Y$ the right $R$-module morphisms

$$
R^{\left(n_{1}, \ldots, n_{k}\right)} \longrightarrow Y
$$

coincide with the symmetric spectra morphisms

$$
S^{n_{1}} \vee \cdots \vee S^{n_{k}} \longrightarrow Y
$$

hence it becomes obvious that $\bmod _{f}\left(\pi_{*} R\right)$ is the homotopy category of $\bmod _{f}(R)$ and the projection functor is given by taking homotopy groups $p=\pi_{*}$. 
The group of self-tracks of a map $X \rightarrow Y$ between fibrant-cofibrant right $R$ modules coincides with the abelian group of homotopy classes $[\Sigma X, Y]_{R}$ in the category of right $R$-modules, compare BD89. If $X$ and $Y$ are weakly equivalent to free right $R$-modules then this abelian group is canonically isomorphic to $\operatorname{Hom}_{\pi_{*} R}\left(\pi_{*} X, \pi_{*} Y \otimes_{\pi_{*} R} \Sigma^{-1} \pi_{*} R\right)$ by the same argument as before. The axioms of a linear track extension are checked in [BD89] in a more general setting.

The lemma above yields a universal Toda bracket associated to a connective ring spectrum $R$

$$
\left\langle\bmod _{f}(R)\right\rangle \in H M L^{*}\left(\pi_{*} R, \Sigma^{-1} \pi_{*} R\right) .
$$

This universal Toda bracket determines all Toda brackets in $\bmod _{f}(R)$, see BD89, i.e. all matric Toda brackets in $R$, compare May69. It has also been studied by Sagave from a different perspective, see Sag06. The goal of this section is the construction of a small algebraic model for this universal Toda bracket. For this we will consider universal Toda brackets associated to quadratic pair algebras.

Given a quadratic pair algebra $B$, in order to obtain a convenient groupoidenriched category of right $B$-modules we need to consider weak morphisms.

Definition 11.3. A weak morphism $f: C \rightarrow D$ between quadratic pair modules consists of group homomorphisms

$$
\begin{gathered}
f_{0}: C_{0} \longrightarrow C_{0}, \\
f_{1}: C_{1} \longrightarrow D_{1}, \\
f_{e e}: C_{e e} \longrightarrow D_{e e}
\end{gathered}
$$

such that

$$
\begin{aligned}
f_{0} \partial & =\partial f_{1}, \\
f_{1} P & =P f_{e e} \\
f_{e e}(-\mid-)_{H} & =\left(f_{0} \mid f_{0}\right)_{H}, \\
f_{e e} T & =T f_{e e} .
\end{aligned}
$$

Weak morphisms were introduced in BM06c]. The functors $h_{0}$ and $h_{1}$ in (2.1) obviously extend to the category wqpm of quadratic pair modules and weak morphisms.

A weak morphism $f: M \rightarrow N$ between right $B$-modules is a collection of weak morphisms $f_{n}: M_{n, *} \rightarrow N_{n, *}, n \geq 0$, which are strictly compatible with the action of $B$, i.e. given $m_{i} \in M_{p, i}$ and $b_{i} \in B_{q, i}$ for $i=0,1, e e$,

$$
\begin{aligned}
f_{p+q, 0}\left(m_{0} \cdot b_{0}\right) & =f_{p, 0}\left(m_{0}\right) \cdot b_{0}, \\
f_{p+q, 1}\left(m_{0} \cdot b_{1}\right) & =f_{p, 0}\left(m_{0}\right) \cdot b_{1}, \\
f_{p+q, 1}\left(m_{1} \cdot b_{0}\right) & =f_{p, 1}\left(m_{1}\right) \cdot b_{0}, \\
f_{p+q, e e}\left(m_{e e} \cdot b_{e e}\right) & =f_{p, e e}\left(m_{e e}\right) \cdot b_{e e} .
\end{aligned}
$$

The reader can easily check that right $B$-modules together with weak morphisms form a category that we denote by $\operatorname{wmod}(B)$. The ordinary category of right $B$ $\operatorname{modules} \bmod (B)$ is a subcategory of $\operatorname{wmod}(B)$ with the same objects.

The category $\operatorname{wmod}(B)$ is also a groupoid-enriched category with the tracks (i.e. 2-morphisms) defined as follows.

Definition 11.4. Let $f, g: C \rightarrow D$ be weak morphisms between quadratic pair modules. A track $\alpha: f \Rightarrow g$ is a function $\alpha: C_{0} \rightarrow D_{1}$ satisfying the equations, 


$$
\begin{aligned}
x, y \in C_{0}, z \in C_{1}, & \\
\alpha(x+y) & =\alpha(x)+\alpha(y)+P\left(-f_{0}(x)+g_{0}(x) \mid f_{0}(y)\right)_{H}, \\
g_{0}(x) & =f_{0}(x)+\partial \alpha(x), \\
g_{1}(z) & =f_{1}(z)+\alpha \partial(z) .
\end{aligned}
$$

This definition of track was given in BM06c, where we show that the category wqpm of quadratic pair modules and weak morphisms is a groupoid-enriched category. The subcategory $\mathbf{q p m}$ is also groupoid-enriched. Tracks in qpm are just tracks in wqpm between morphisms in qpm.

The vertical composition of tracks

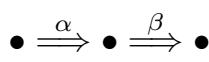

is defined by addition $(\beta \square \alpha)(x)=\alpha(x)+\beta(x)$. The horizontal composition of tracks and maps as in

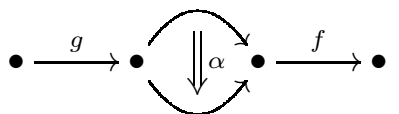

is defined as $(f \alpha)(x)=f_{1} \alpha(x)$ and $(\alpha g)(x)=\alpha g_{0}(x)$. The identity track $0_{f}^{\square}: f \Rightarrow f$ (also called trivial track) is always defined as $\left(0_{f}^{\square}\right)(x)=0$.

Let $B$ be a quadratic pair algebra and let $f, g: M \rightarrow N$ be weak right $B$-module morphisms. A track $\alpha: f \Rightarrow g$ in $\bmod (B)$ is collection of tracks $\alpha_{n}: f_{n} \Rightarrow g_{n}$ between the quadratic pair module morphisms $f_{n}, g_{n}: M_{n} \rightarrow N_{n}$ such that for any $x \in M_{n, 0}$ and $a \in B_{m, 0}$

$$
\alpha_{n+m}(x \cdot a)=\alpha_{n}(x) \cdot a+P\left(\left(-f_{n, 0}(x)+g_{n, 0}(x) \mid f_{n, 0}(x)\right)_{H} \cdot H(a)\right) .
$$

We leave it to the reader to check that these tracks with vertical and horizontal composition defined as above endow $\operatorname{wmod}(B)$ with a groupoid-enriched category structure.

Remark 11.5. The groupoid-enriched category qpm was studied in BM06c, BM07. The enrichment extends in the obvious way to the category of $\mathbb{N}$-graded objects $\mathbf{q p m}^{\mathbb{N}}$ and restricts to the subcategory of 0 -good objects $X$ in $\mathbf{q p m}_{H}^{\mathbb{N}}$ which is monoidal. In this monoidal category tracks can be interpreted as morphisms from the cylinder object $\mathbb{I} \odot X$ defined by the interval quadratic pair module $\mathbb{I}$, see [BM07, Lemma 5.3]. If $B$ is a 0 -good quadratic pair algebra the cylinder of a 0 -good right $B$-module is again a right $B$-module in a natural way, so we can define tracks as right $B$-module morphisms from the cylinder. In this way we obtained the formulas for tracks in Definition 11.4 above. This also works for weak morphisms.

Let $B$ be a quadratic pair algebra. A free right $B$-module is a $B$ module of the form

$$
B^{\left(n_{1}, \ldots, n_{k}\right)}=\left(\left(\overline{\mathbb{Z}}_{n i l}, n_{1}\right) \vee \cdots \vee\left(\overline{\mathbb{Z}}_{n i l}, n_{k}\right)\right) \odot B, \quad k \geq 0, n_{1}, \ldots, n_{k} \geq 0 .
$$

Here $\left(\overline{\mathbb{Z}}_{n i l}, m\right)$ denotes the $\mathbb{N}$-graded quadratic pair module given by $\overline{\mathbb{Z}}_{n i l}$ concentrated in degree $m$ and $\vee$ is the coproduct. We denote by $\operatorname{wmod}_{f}(B)$ the full groupoid-enriched subcategory of $\operatorname{wmod}(B)$ consisting of free right $B$-modules.

Lemma 11.6. Let $B$ be a quadratic pair algebra. Then the category $\operatorname{wmod}_{f}(B)$ is a linear track extension of $\bmod _{f}\left(h_{0} B\right)$ by $\operatorname{Hom}_{h_{0} B}\left(-,-\otimes_{h_{0} B} h_{1} B\right)$.

The proof of this lemma will be given below.

Lemma 11.6 yields a universal Toda bracket in Mac Lane cohomology for any quadratic pair algebra $B$

$$
\left\langle\operatorname{wmod}_{f}(B)\right\rangle \in H M L^{*}\left(h_{0} B, h_{1} B\right) .
$$

The following theorem is the main result of this section. 
Theorem 11.7. Let $R$ be a connective ring spectrum. If we use the isomorphisms in Theorem 6.4 as identifications then the universal Toda brackets associated to the ring spectrum $R$ and to the quadratic pair algebra $\pi_{*, *} R$ coincide, that is:

$$
\left\langle\bmod _{f}(R)\right\rangle=\left\langle\operatorname{wmod}_{f}\left(\pi_{*, *} R\right)\right\rangle .
$$

We prove Theorem 11.7 at the end of this section.

Remark 11.8. In BJP06, Theorem 2.2.1] it is proved in the ungraded setting that any element in Mac Lane cohomology $H M L^{3}(A, M)$ is represented by a quadratic pair algebra $B$ with $h_{0} B \cong A$ and $h_{1} B \cong M$. Such a quadratic pair algebra is also termed a "crossed extension of $A$ by $M$ in the symmetric monoidal category of square groups", see the Addendum of [BJP06, Theorem 2.2.1]. Since for a connective ring spectrum $R$ the topologically defined category $\bmod _{f}(R)$ represents an element in $H M L^{3}\left(\pi_{*} R, \Sigma^{-1} \pi_{*} R\right)$ the result in BJP06] makes it plausible that, in fact, the element $\left\langle\bmod _{f}(R)\right\rangle$ should also be represented by a quadratic pair algebra. By Theorem 11.7 above we construct such a quadratic pair algebra, given by $\pi_{*, *} R$, even in a functorial way.

In order to prove Lemma 11.6 we need the following results.

Lemma 11.9. There are natural isomorphisms

$$
\begin{aligned}
h_{0}\left(B^{\left(n_{1}, \ldots, n_{k}\right)}\right) & \cong\left(h_{0} B\right)^{\left(n_{1}, \ldots, n_{k}\right)}, \\
h_{1}\left(B^{\left(n_{1}, \ldots, n_{k}\right)}\right) & \cong\left(h_{0} B\right)^{\left(n_{1}, \ldots, n_{k}\right)} \otimes_{h_{0} B} h_{1} B .
\end{aligned}
$$

Lemma 11.9 follows easily from the following one.

Lemma 11.10. For any quadratic pair module $C$ there are natural isomorphisms

$$
\begin{aligned}
& h_{0}\left(\left(\overline{\mathbb{Z}}_{n i l} \vee \cdot \stackrel{k}{*} \vee \overline{\mathbb{Z}}_{n i l}\right) \odot C\right) \cong h_{0} C \oplus \stackrel{k}{*} \oplus h_{0} C, \\
& h_{1}\left(\left(\overline{\mathbb{Z}}_{n i l} \vee \cdot \stackrel{k}{*} \vee \overline{\mathbb{Z}}_{n i l}\right) \odot C\right) \cong h_{1} C \oplus \stackrel{k}{*} \oplus h_{1} C .
\end{aligned}
$$

Proof. There is an exact sequence of square groups in the sense of [BJP05]

$$
h_{1} C \hookrightarrow C_{(1)} \stackrel{\partial}{\longrightarrow} C_{(0)} \rightarrow h_{0} C .
$$

Given a pointed set $E$ the square group $\mathbb{Z}_{n i l}[E]$ in (5.1) is the coproduct of copies of the unit element $\mathbb{Z}_{n i l}$ for the tensor product of square groups indexed by $E$ excluding the base-point. Therefore by using the definition in [BM07] of the tensor product in qpm we see that the quadratic pair module

$$
\left(\overline{\mathbb{Z}}_{n i l} \vee \cdots \vee \overline{\mathbb{Z}}_{n i l}\right) \odot C
$$

coincides with

$$
\mathbb{Z}_{n i l}[\underline{k}] \odot C_{(1)} \stackrel{\mathbb{Z}_{n i l}[\underline{k}] \odot \partial}{\longrightarrow} \mathbb{Z}_{n i l}[\underline{k}] \odot C_{(0)}
$$

where $\underline{k}=\{0,1, \ldots, k\}$ pointed at 0 .

The square groups in (5.1) are flat for the tensor product of square groups. This follows easily from [BJP05, Corollary 39] since the unit object $\mathbb{Z}_{n i l}$ is obviously flat. Moreover, it is easy to check by using the generatos and relations for the tensor product of square groups in BJP05] that for any abelian group $A$ there is a natural isomorphism $\mathbb{Z}_{\text {nil }}[\underline{k}] \odot A \cong \mathbb{Z}[\underline{k}] \otimes A$, and hence the lemma follows.

The following lemma is a simple exercise.

Lemma 11.11. Let $C$ be any quadratic pair module. Weak morphisms $f: \overline{\mathbb{Z}}_{n i l} \rightarrow C$ are in bijective correspondence with the elements of $C_{0}$. The correspondence sends $f$ to $f_{0}(1)$ for $1 \in \mathbb{Z}=\left(\overline{\mathbb{Z}}_{n i l}\right)_{0}$. Moreover, given two morphisms $f, g: \overline{\mathbb{Z}}_{n i l} \rightarrow C$ the 
set of tracks $\alpha: f \Rightarrow g$ is in bijection with the subset $\partial^{-1}\left(-f_{0}(1)+g_{0}(1)\right) \subset C_{1}$. The bijection sends $\alpha$ to $\alpha(1)$.

Now we are ready to prove Lemma 11.6 .

Proof of Lemma 11.6. Weak right $B$-module morphisms

$$
B^{\left(n_{1}, \ldots, n_{k}\right)} \longrightarrow B^{\left(m_{1}, \ldots, m_{l}\right)}
$$

and tracks between them are in bijection with weak morphisms between graded quadratic pair modules

$$
\left(\overline{\mathbb{Z}}_{n i l}, n_{1}\right) \vee \cdots \vee\left(\overline{\mathbb{Z}}_{n i l}, n_{k}\right) \longrightarrow B^{\left(m_{1}, \ldots, m_{l}\right)}
$$

and tracks between them. Hence Lemma[11.6 follows from Lemmas 11.9 and 11.11 The projection onto the homotopy category is $h_{0}: \operatorname{wmod}_{f}(B) \rightarrow \bmod _{f}\left(h_{0} B\right)$.

We finally need the following result.

Lemma 11.12. Let $R$ be a connective ring spectrum. The restriction of the functor $\pi_{*, *}$ in Theorem 7.4 to the full subcategory of connective fibrant-cofibrant right $R$ modules can be extended to a groupoid-enriched functor.

Proof. The functor $\pi_{*, *}$ in Theorem 7.4 is constructued in Section 16 by using secondary homotopy groups of spectra. Secondary homotopy groups of spectra are defined as colimits of additive secondary homotopy groups of spaces which are groupoid-enriched functors, see BM06a, BM06c. Therefore secondary homotopy groups of spectra take homotopies to tracks between quadratic pair modules, and in particular the functor $\pi_{*, *}$ in Theorem 7.4 becomes a groupoid-enriched functor on connective fibrant-cofibrant right $R$-modules.

Now we are ready to prove Theorem 11.7

Proof of Theorem 11.7. Recall that a pseudofunctor between groupoid-enriched categories $\varphi: \mathbf{C} \rightarrow \mathbf{B}$ is an assignment of objects, maps and tracks which preserves horizontal composition and identity morphisms only up to certain given tracks

$$
\varphi_{f, g}: \varphi(f) \varphi(g) \Rightarrow \varphi(f g) \text { and } \varphi_{X}: \varphi\left(1_{X}\right) \Rightarrow 1_{\varphi(X)} .
$$

These tracks must satisfy well-known coherence and naturality properties. Track functors are pseudofunctors where these tracks are trivial. Similarly there is a definition of pseudonatural transformation $\alpha: \varphi \Rightarrow \psi$ between pseudofunctors $\varphi, \psi: \mathbf{C} \rightarrow$ B given by maps $\alpha_{X}: \varphi(X) \rightarrow \psi(X)$ such that the usual square does not commute, but for any map $f: X \rightarrow Y$ there is a track $\alpha_{f}: \psi(f) \alpha_{X} \Rightarrow \alpha_{Y} \varphi(f)$ satisfying coherence and naturality properties.

Two linear track extensions $\mathbf{B}, \mathbf{C}$ of $\mathbf{A}$ by $D$ have the same universal Toda bracket if and only if there is a pseudofunctor $\varphi: \mathbf{C} \rightarrow \mathbf{B}$ such that for any morphism $f: X \rightarrow Y$ in $\mathbf{C}$ the equality $p_{\mathbf{B}} \varphi(f)=p_{\mathbf{C}}(f)$ holds, and for any $x \in D(X, Y)$ the equation $\sigma_{\varphi(f)}(x)=\varphi \sigma_{f}(x)$ is satisfied, compare BM05.

We now prove Theorem 11.7 by constructing an appropriate pseudofunctor

$$
\varphi: \bmod _{f}(R) \longrightarrow \operatorname{wmod}_{f}\left(\pi_{*, *} R\right) .
$$

The functor sending a fibrant replacement of a free right $R$-module $X$ to $\pi_{*, *} X$, see Theorem 7.4, takes values in the category of right $\pi_{*, *} R$-modules, but not on the full subcategory $\operatorname{wmod}_{f}\left(\pi_{*, *} R\right)$. However, the fixed trivial cofibration $R^{\left(n_{1}, \ldots, n_{k}\right)} \stackrel{\sim}{\longmapsto} X$ together with Theorem 15.17 and Remark 15.2 give rise to a right $\pi_{*, *} R$-module morphism

$$
\alpha_{X}: \varphi(X)=\left(\pi_{*, *} R\right)^{\left(n_{1}, \ldots, n_{k}\right)} \longrightarrow \pi_{*, *} X
$$


see Remark 16.1 for details, which induces isomorphisms in $h_{0}$ and $h_{1}$, compare Lemma 11.9. Now it is easy to check that for any map $f: X \rightarrow Y$ between free right $R$-modules we can choose a morphism $\varphi(f)$ and a track $\alpha_{f}$ as in diagram

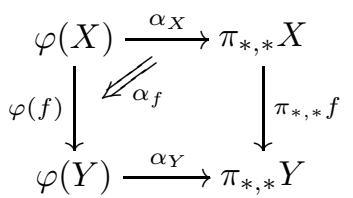

Since $\pi_{*, *}$ is groupoid-enriched functor by Lemma 11.12 and $\alpha_{X}$ and $\alpha_{Y}$ induce isomorphisms on $h_{0}$ and $h_{1}$ then by categorical nonsense there is a unique pseudofunctor $\varphi$ defined on objects and maps as above such that $\alpha: \varphi \Rightarrow \pi_{*, *}$ is a pseudonatural transformation. This pseudofunctor proves the theorem.

\section{SyMmetriC SPECTRA}

We work in the symmetric monoidal model category Top* of pointed (compactly generated) spaces, see [Hov99, 4.2.12].

A spectrum $X$ is a sequence of pointed spaces $X_{0}, X_{1}, \ldots, X_{n}, \ldots$ together with pointed maps $\sigma_{n}: S^{1} \wedge X_{n} \rightarrow X_{1+n}$ called structure maps. A morphism of spectra $f: X \rightarrow Y$ is a sequence of pointed maps $f_{n}: X_{n} \rightarrow Y_{n}$ with $\sigma_{n} f_{n}=f_{1+n} \sigma_{n}$. The category of spectra will be denoted by $\mathbf{S p}^{\mathbb{N}}$.

An $\Omega$-spectrum $X$ is a spectrum such that the adjoints $\operatorname{ad}\left(\sigma_{n}\right): X_{n} \rightarrow \Omega X_{1+n}$ of the structure maps $\sigma_{n}$ are weak equivalences for all $n \geq 0$.

A symmetric spectrum is a more structured notion of spectrum defined in the following way. An object $X$ in the category (Top $\left.{ }^{*}\right)^{\text {Sym }}$ of symmetric sequences in Top* ${ }^{*}$ is a sequence of spaces $X_{0}, X_{1}, \ldots, X_{n}, \ldots$ such that $X_{n}$ carries a left action of the symmetric group $\operatorname{Sym}(n)$. A morphism $X \rightarrow Y$ of symmetric sequences is a sequence of equivariant maps $X_{n} \rightarrow Y_{n}$. The category of symmetric sequences has a symmetric monoidal structure with the tensor product $X \otimes Y$ characterized by the existence of a natural isomorphism

$$
\operatorname{Hom}_{\left(\text {Top }^{*}\right)} \operatorname{Sym}(X \otimes Y, Z) \cong \prod_{p, q \geq 0} \operatorname{Hom}_{\times}\left(X_{p} \wedge Y_{q}, Z_{p+q}\right): f \mapsto f_{p, q}
$$

Here $\mathrm{Hom}_{\times}$is the set of equivariant morphisms with respect to the cross product homomorphism $\times$ in (8.6). The symmeytry isomorphism $X \otimes Y \cong Y \otimes X$ is given by the maps

$$
X_{p} \wedge Y_{q} \cong Y_{q} \wedge X_{p} \stackrel{\left(1_{Y \otimes X}\right)_{q, p}}{\longrightarrow}(Y \wedge X)_{q+p} \stackrel{\tau_{q, p}}{\longrightarrow}(Y \wedge X)_{p+q}
$$

The shuffle permutation $\tau_{q, p}$ was already considered in Definition 9.1.

The sphere spectrum $S$ is the symmetric sequence $S$ given by the spheres $S^{0}$, $S^{1}, \ldots, S^{n}, \ldots$, where $\operatorname{Sym}(n)$ acts on $S^{n}=S^{1} \wedge \cdots \wedge S^{1}$ by permutation of coordinates. This symmetric sequence is a commutative monoid with multiplication $\mu: S \otimes S \rightarrow S$ given by $\mu_{p, q}: S^{p} \wedge S^{q}=S^{p+q}$. The category $\mathbf{S p}^{\Sigma}$ of symmetric spectra is the category of left $S$-modules. The structure maps $\sigma_{1, n}: S^{1} \wedge X_{n} \rightarrow X_{1+n}$ of the underlying spectrum of a symmetric spectrum $X$ are defined by the left action $\sigma: S \otimes X \rightarrow X$.

Since the monoid $S$ is commutative the category $\mathbf{S p}^{\Sigma}$ is symmetric monoidal for the tensor product $\otimes_{S}$ also called smash product and denoted by $\wedge$. The smash product of symmetric spectra $X \wedge Y$ is the coequalizer of the two multiplications $X \otimes S \otimes Y \rightrightarrows X \otimes Y$. The smash product $X \wedge Y$ comes equipped with natural maps, $p, q \geq 0$,

$$
\jmath_{p, q}: X_{p} \wedge Y_{q} \longrightarrow(X \wedge Y)_{p+q}
$$


satisfying a universal property which characterizes $X \wedge Y$ as a symmetric spectrum, see Sch04.

The category $\mathbf{S p}^{\mathbb{N}}$ of spectra is a stable model category, see [MMSS01, 4.1 and 9], while $\mathbf{S p}^{\Sigma}$ is in addition a symmetric monoidal model category, see MMSS01, 4.2 and 9]. We wish to emphasize that among the possible model category structures on $\mathbf{S p}^{\mathbb{N}}$ and $\mathbf{S p}^{\Sigma}$ we have chosen to work with the stable model structure defined in [MMSS01, 9] since with this structure fibrant objects coincide in both cases with the $\Omega$-spectra.

Symmetric sequences and symmetric spectra defined in this way are available over monoidal categories more general than Top*, see Hov01]. They can also be defined by using right actions of symmetric groups instead of left actions.

\section{SECONDARY HOMOTOPY GROUPS OF SPACES}

A groupoid-enriched category, also termed track category, is a 2-category where all 2-morphisms are invertible with respect to vertical composition. We also call tracks to the 2-morphisms in track categories. Horizontal composition is denoted by juxtaposition, and we use the symbol $\square$ for the vertical composition. The vertical inverse of a track $\alpha$ is $\alpha^{\boxminus}$. Identity morphisms are denoted by 1 , and the symbol $0^{\square}$ is used for identity tracks. Track functors are 2-functors between track categories. The category Top* is a track category where a track between two maps is a relative homotopy class of homotopies between them. Similarly for the category of fibrantcofibrant spectra or symmetric spectra, compare [Bau89]. For the convenience of the reader we recall from BM06a, BM06c the following definition.

Definition 13.1. Let $n \geq 3$. For a pointed space $X$ we define the additive secondary homotopy group, $\Pi_{n, *} X$ which is a 0 -free quadratic pair module with $\Pi_{n,(0)} X=\mathbb{Z}_{n i l}\left[\Omega^{n} X\right]$ where $\Omega^{n} X$ is the discrete pointed set of maps $S^{n} \rightarrow X$ in Top*.

We describe the group $\Pi_{n, 1} X$ and the homomorphisms $P$ and $\partial$ as follows. Given a pointed set $E$ we denote by $\vee_{E} S^{n}$ to the $n$-fold suspension of $E$ in Top* ${ }^{*}$ It is a one-point union of $n$-spheres indexed by the set $E-\{*\}$. The group $\Pi_{n, 1} X$ is given by the set of equivalence classes $[f, F]$ represented by a map $f: S^{1} \rightarrow \vee_{\Omega^{n} X} S^{1}$ and a track in Top* of the form:

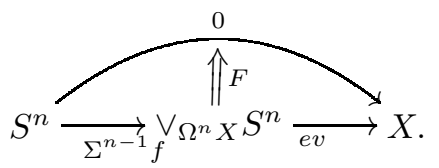

Here the map ev: $\vee_{\Omega^{n} X} S^{n} \rightarrow X$ is the obvious evaluation map.

The equivalence relation $[f, F]=[g, G]$ holds provided there is a track

$$
N: \Sigma^{n-1} f \Rightarrow \Sigma^{n-1} g
$$

with trivial Hopf invariant $\operatorname{Hopf}(N)=0$ in the sense of (13.3) below such that the composite track in the following diagram is the trivial track.

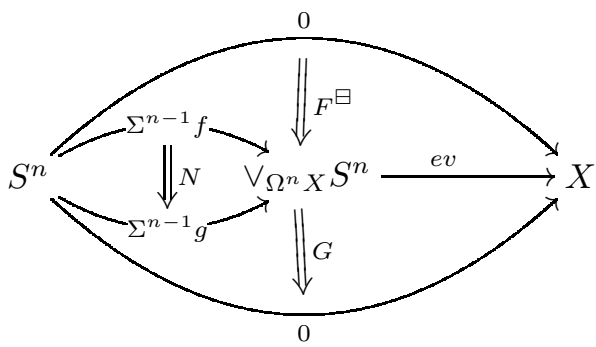


The element $\partial[f, F] \in\left\langle\Omega^{n} X\right\rangle_{n i l}$ is given by the image of the generator $1 \in \mathbb{Z} \cong \pi_{1} S^{1}$ under the homomorphism

$$
\pi_{1} f: \pi_{1} S^{1} \longrightarrow \pi_{1}\left(\vee_{\Omega^{n} X} S^{1}\right) \cong\left\langle\Omega^{n} X\right\rangle .
$$

Let $I_{+}$be the unit interval $I=[0,1]$ with an outer base-point and let $I X=$ $I_{+} \wedge X$ be the reduced cylinder of $X$ in Top*. The Hopf invariant Hopf $(N)$ of a track $N: \Sigma^{n-1} f \Rightarrow \Sigma^{n-1} g$ with $f, g: S^{1} \rightarrow \vee_{E} S^{1}$ is defined in [BM06a] 3.3 by the homomorphism

$$
H_{2}\left(I S^{1}, S^{1} \vee S^{1}\right) \stackrel{\operatorname{ad}(N)_{*}}{\longrightarrow} H_{2}\left(\Omega^{n-1}\left(\vee_{E} S^{n}\right), \vee_{E} S^{1}\right) \cong \hat{\otimes}^{2} \mathbb{Z}[E],
$$

which carries the generator $1 \in \mathbb{Z} \cong H_{2}\left(I S^{1}, S^{1} \vee S^{1}\right)$ to $\operatorname{Hopf}(N)$. Here the isomorphism is induced by the Pontrjagin product and $a d(N)_{*}$ is the homomorphism induced in homology by the adjoint of

$$
S^{n-1} \wedge I_{+} \wedge S^{1} \cong I_{+} \wedge S^{n} \stackrel{N}{\rightarrow} \vee_{E} S^{n} .
$$

The reduced tensor square $\hat{\otimes}^{2}$ in (13.3) is defined for any abelian group $A$ as the quotient

$$
\hat{\otimes}^{2} A=\frac{A \otimes A}{a \otimes b+b \otimes a \sim 0}
$$

and $\bar{\sigma}: \otimes^{2} A \rightarrow \hat{\otimes}^{2} A$ denotes the natural projection. If $f, g: \vee_{E^{\prime}} S^{1} \rightarrow \vee_{E} S^{1}$ are maps from a coproduct the Hopf invariant $\operatorname{Hopf}(N): \mathbb{Z}\left[E^{\prime}\right] \rightarrow \hat{\otimes}^{2} \mathbb{Z}[E]$ is a homomorphism defined on the basis $E^{\prime}$ by the Hopf invariants of the restriction of $N$ to the components of $\vee_{E^{\prime}} S^{n}$. We refer the reader to [BM06a, 3] for the elementary properties of the invariant Hopf.

The equivalence classes $[f, F]$ define $\Pi_{n, 1} X$ as a set. The group structure of $\Pi_{n, 1} X$ is induced by the comultiplication $\mu: S^{1} \rightarrow S^{1} \vee S^{1}$, compare BM06a 4.4.

We now define the homomorphism $P$. Consider the diagram

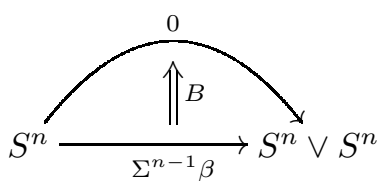

where $\beta: S^{1} \rightarrow S^{1} \vee S^{1}$ is given such that $\left(\pi_{1} \beta\right)(1)=-a-b+a+b$ is the commutator of the canonical generators of $\pi_{1}\left(S^{1} \vee S^{1}\right)$, i.e. $a$ corresponds to the inclusion of the first sphere and $b$ to the inclusion of the second one. The track $B$ is any track with $H o p f(B)=-\bar{\sigma}(a \otimes b)$. Given $x \otimes y \in \otimes^{2} \mathbb{Z}\left[\Omega^{n} X\right]$ let $\tilde{x}, \tilde{y}: S^{1} \rightarrow \vee_{\Omega^{n} X} S^{1}$ be maps such that the images of $\left(\pi_{1} \tilde{x}\right)(1)$ and $\left(\pi_{1} \tilde{y}\right)(1)$ in $\mathbb{Z}\left[\Omega^{n} X\right]$ are $x$ and $y$, respectively. Then the diagram

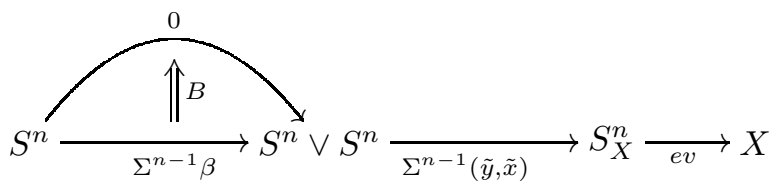

represents $P(x \otimes y) \in \Pi_{n, 1} X$. This completes the definition of the quadratic pair module $\Pi_{n, *} X$ for $n \geq 3$.

Additive secondary homotopy groups are defined in BM06c also for $n=0,1,2$. In this way we get for $n \geq 0$ a functor

$$
\Pi_{n, *}: \text { Top }^{*} \longrightarrow \mathbf{q p m}
$$

which is actually a track functor. Moreover, $\Pi_{n,(0)} X=\mathbb{Z}_{n i l}\left[\Omega^{n} X\right]$ so that $\Pi_{n, *}$ takes values in the full subcategory $\mathbf{q p m}_{H}$ of 0 -free, and hence 0 -good, quadratic pair modules. 
Remark 13.5. By [BM06a, 4.16] $\Pi_{n, *} S^{n}$ is quasi-isomorphic to $\overline{\mathbb{Z}}_{n i l}$. The quasiisomorphism $\imath_{n, 0}: \overline{\mathbb{Z}}_{n i l} \rightarrow \Pi_{n, *} S^{n}$ is defined by $\imath_{n, 0}(1)=1_{S^{n}} \in\left\langle\Omega^{n} S^{n}\right\rangle_{n i l}$ for $1 \in \mathbb{Z}=\left(\overline{\mathbb{Z}}_{n i l}\right)_{0}$. This quasi-isomorphism is an isomorphism for $n=0$.

The secondary homotopy group $\Pi_{n, *} X$ is endowed with a natural action of the symmetric track group $\operatorname{Sym}_{\square}(n)$, see $[$ BM06c . The underlying action of $\operatorname{Sym}(n)$ on $\Pi_{n,(0)} X=\mathbb{Z}_{n i l}\left[\Omega^{n} X\right]$ is given by the right action of $\operatorname{Sym}(n)$ on the pointed set $\Omega^{n} X$ of maps $S^{n} \rightarrow X$ determined by the left action of $\operatorname{Sym}(n)$ on the $n$-sphere $S^{n}=S^{1} \wedge \cdots \wedge S^{1}$ by permutation of coordinates.

One of the results in BM07 states that the functor

$$
\Pi_{*, *}: \text { Top }^{*} \longrightarrow \mathbf{q p m}_{H}^{\mathbb{N}}
$$

to the category of $\mathbb{N}$-graded 0 -good quadratic pair modules is lax monoidal with multiplication given by the smash product operation for secondary homotopy groups

$$
\Pi_{n, *} X \odot \Pi_{m, *} Y \stackrel{\wedge}{\longrightarrow} \Pi_{n+m, *}(X \wedge Y) .
$$

This morphism is equivariant with respect to the sign group morphism (8.5). The functor $\Pi_{*, *}$ in (13.6) is however non-symmetric. In order to obtain a symmetric version we have to regard $\Pi_{*, *}$ as a functor to the category $\mathbf{q p m}_{H}^{\mathrm{Sym}}$ of enhanced symmetric sequences of 0 -good quadratic pair modules defined in [BM07, 8.14]. The properties of the smash product for secondary homotopy groups yield a lax symmetric monoidal functor

$$
\Pi_{*, *}: \mathbf{T o p}^{*} \longrightarrow \mathbf{q p m}_{H}^{\mathrm{Sym}} \square .
$$

This is the main result in BM07.

There are natural isomorphisms, $n \geq 3$,

$$
\begin{aligned}
& h_{0} \Pi_{n, *} X \cong \pi_{n} X, \\
& h_{1} \Pi_{n, *} X \cong \pi_{n+1} X .
\end{aligned}
$$

Here we use BM06a 5.1. Furthermore, the following property is crucial.

Proposition 13.9. For all $n \geq 3$ the $k$-invariant of $\Pi_{n, *} X$ defined in (5.4) coincides via (13.8) with the homomorphism $\eta^{*}: \pi_{n} X \rightarrow \pi_{n+1} X$ induced by precomposition with $\Sigma^{n-2} \eta$ where $\eta: S^{3} \rightarrow S^{2}$ is the Hopf map.

This follows from BM06a, 8.2].

\section{The PULL-BACK CONSTRUCTION}

Let $C$ be any quadratic pair module. Given a pointed set $E$ and a pointed map $E \rightarrow \operatorname{Ker} H \subset C_{0}$, or equivalently a group homomorphism $\varphi:\langle E\rangle_{n i l} \rightarrow C_{0}$ such that $H \varphi(e)=0$ for any $e \in E$ then the 0 -free quadratic pair module $\varphi^{*} C$ is defined as follows.

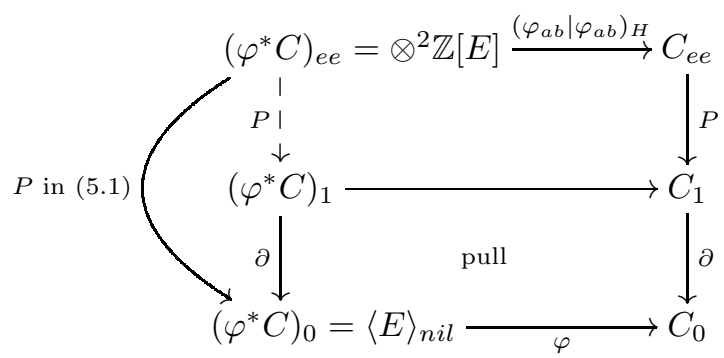

This quadratic pair module comes equipped with a morphism $\varphi_{\#}: \varphi^{*} C \rightarrow C$ in qpm which is given by the horizontal arrows in diagram (14.1). This construction can be straightforwardly extended to the graded setting degreewise. 
Lemma 14.2. In the conditions above the morphism $\varphi_{\#}: \varphi^{*} C \rightarrow C$ induces always an isomorphism on $h_{1}$. It also induces an isomorphism on $h_{0}$ provided the set of elements in $h_{0} C$ comming from $E$ generate the group $h_{0} C$.

Lemma 14.3. Suppose that $G_{\square}$ is a sign group acting on a quadratic pair module $C$ and that the group $G$ acts on $E$ in such a way that $\varphi$ is $G$-equivariant. Then there is a unique action of $G_{\square}$ on $\varphi^{*} C$ such that the induced $G$-action on level 0 is given by the action of $G$ on $E$ and the natural morphism $\varphi_{\#}: \varphi^{*} C \rightarrow C$ is a right $A\left(G_{\square}\right)$-module morphism.

This follows easily from the universal property of a pull-back.

Lemma 14.4. If $C$ is a quadratic pair algebra, $E$ is a pointed graded monoid, and $E \rightarrow \operatorname{Ker} H \subset C_{0}$ is a graded monoid morphism then there is a unique quadratic pair algebra structure on $\varphi^{*} C$ such that the monoid structure of $\left(\varphi^{*} C\right)_{*,(0)}=\mathbb{Z}_{n i l}[E]$ in SG is induced by the monoid structure on $E$ in the sense of [BJP05, Section 12.1] and the natural morphism $\varphi_{\#}: \varphi^{*} C \rightarrow C$ is a morphism of quadratic pair algebras.

This is also a consequence of the universal property of a pull-back. In can be extended to the $E_{\infty}$-case.

Lemma 14.5. Suppose that $C$ is an $E_{\infty}$-quadratic pair algebra, $E$ is a pointed graded monoid such that the symmetric group $\operatorname{Sym}(n)$ acts on $E_{n}$ in such a way that the multiplications $E_{n} \wedge E_{m} \rightarrow E_{n, m}$ are equivariant with respect to the cross product homomorphisms in (8.6), and $E \rightarrow$ Ker $H$ is a degreewise equivariant graded monoid morphism. Then there is a unique $E_{\infty}$-quadratic pair algebra structure on $\varphi^{*} C$ such that the monoid structure of $\left(\varphi^{*} C\right)_{*,(0)}=\mathbb{Z}_{n i l}[E]$ in $\mathbf{S G}$ is induced by the monoid structure on $E$ in the sense of [BJP05, Section 12.1], the action of $\operatorname{Sym}(n)$ on $\left(\varphi^{*} C\right)_{n,(0)}=\mathbb{Z}_{n i l}\left[E_{n}\right]$ is given by the action on $E_{n}$, and the natural morphism $\varphi_{\#}: \varphi^{*} C \rightarrow C$ is a morphism of $E_{\infty}$-quadratic pair algebras.

\section{SECONDARY HOMOTOPY GROUPS OF SPECTRA}

Secondary homotopy groups of spaces introduced in the previous section can be used to define secondary homotopy groups of spectra which are the crucial tools in this paper.

Given a space $X$ and a pointed map $E \rightarrow \Omega^{n} X$ inducing a homomorphism $\varphi:\langle E\rangle_{n i l} \rightarrow\left\langle\Omega^{n} X\right\rangle_{n i l}$, we write

$$
\Pi_{n, *}(X \mid E)=\varphi^{*} \Pi_{n, *} X
$$

for the pull-back construction defined in (14.1).

Definition 15.1. Let $X$ be now a spectrum. The secondary homotopy group $\pi_{n, *} X$, $n \geq 0$, is defined as the colimit in $\mathbf{q p m}$

$$
\pi_{n, *} X=\operatorname{colim}_{k \geq 0} \Pi_{k+n, *}\left(X_{k} \mid \Omega^{n} X_{0}\right) .
$$

Here the map $\Omega^{n} X_{0} \rightarrow \Omega^{k+n} X_{k}$ is obtained by taking $\Omega^{n}$ on the adjoint of the structure map $\sigma_{k, 0}: S^{k} \wedge X_{0} \rightarrow X_{k}$, i.e. it sends an $n$-loop $f: S^{n} \rightarrow X_{0}$ to the composite

$$
S^{k+n} \stackrel{S^{k} \wedge f}{\longrightarrow} S^{k} \wedge X_{0} \stackrel{\sigma_{k, 0}}{\longrightarrow} X_{k} .
$$

The bonding morphisms of the directed system

$$
\Pi_{k+n, *}\left(X_{k} \mid \Omega^{n} X_{0}\right) \longrightarrow \Pi_{1+k+n, *}\left(X_{1+k} \mid \Omega^{n} X_{0}\right)
$$

are defined to be the identity on levels 0 and $e e$, so $\pi_{n,(0)} X=\mathbb{Z}_{n i l}\left[\Omega^{n} X_{0}\right]$ and $\pi_{n, *} X$ is 0 -free, and hence 0 -good. On the 1 -level the bonding morphism is defined 
by the composite in qpm

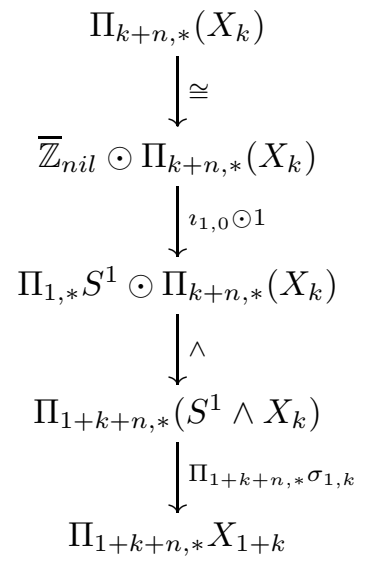

Here the first isomorphism is the unit isomorphism for the symmetric monoidal structure $\odot$ in qpm. In the second arrow we use the morphism $\imath_{1,0}$ in Remark 13.5. The morphism $\wedge$ is the smash product for secondary homotopy groups of spaces constructed in BM07.

Remark 15.2. The quadratic pair module $\pi_{n, *} S^{n}$ is quasi-isomorphic to $\overline{\mathbb{Z}}_{n i l}$. The quasi-isomorphism $\imath_{n, 0}: \overline{\mathbb{Z}}_{n i l} \rightarrow \pi_{n, *} S^{n}$ is defined by $\imath_{n, 0}(1)=1_{S^{n}} \in\left\langle\Omega^{n} S^{n}\right\rangle_{n i l}$, as in Remark 13.5. This quasi-isomorphism is an isomorphism for $n=0$. This remark follows for instance from Proposition 15.4 below.

The symmetric track group $\operatorname{Sym}_{\square}(n)$ acts on $\Pi_{k+n, *}\left(X_{k} \mid \Omega^{n} X_{0}\right)$, see Lemma 14.3. The induced action of the symmetric group $\operatorname{Sym}(n)$ at level 0 is given by the action on $\Omega^{n} X_{0}$ considered in the previous section. The bonding morphisms of the directed system are right $A\left(\operatorname{Sym}_{\square}(n)\right)$-module morphisms, therefore $\pi_{n, *} X$ carries a natural action of $\operatorname{Sym}_{\square}(n)$.

For any $\Omega$-spectrum $X$ we derive from (13.8) and Lemma 14.2 the natural isomorphisms, $n \geq 0$,

$$
\begin{aligned}
& h_{0} \pi_{n, *} X \cong \pi_{n} X, \\
& h_{1} \pi_{n, *} X \cong \pi_{n+1} X .
\end{aligned}
$$

The following proposition follows from Proposition 13.9 .

Proposition 15.4. Let $X$ be an $\Omega$-spectrum. The $k$-invariant of $\pi_{n, *} X$ coincides via the isomorphisms (15.3) with the homomorphism $\eta: \pi_{n} X \rightarrow \pi_{n+1} X$ induced by multiplication with the stable Hopf map $\eta \in \pi_{1} S$.

The projection to the colimit yields a natural morphism in qpm

$$
\phi_{n}: \Pi_{n, *} X_{0} \longrightarrow \pi_{n, *} X
$$

In case $X$ is an $\Omega$-spectrum $\phi_{n}$ is levelwise surjective for $n=0,1$ and an isomorphism for $n \geq 2$. The next result is a crucial ingredient for the main theorems in this paper.

Theorem 15.5. Let $X, Y, Z$ be symmetric spectra which are $\Omega$-spectra and let $f: X \wedge Y \rightarrow Z$ be a morphism in $\mathbf{S p}^{\Sigma}$. Then for all $n, m \geq 0$ there is a unique 
morphism $\tilde{f}_{n, m}$ in $\mathbf{q p m}$ for which the following diagram commutes.

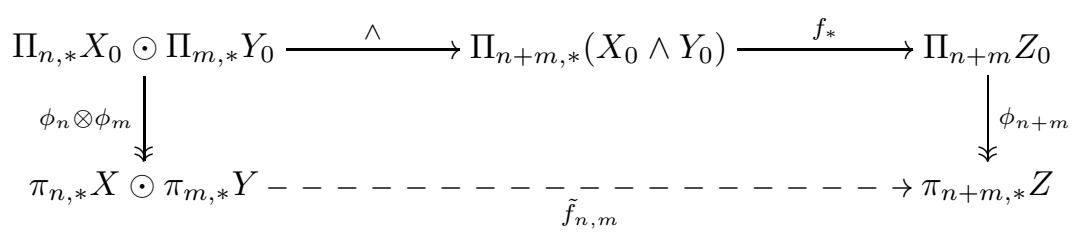

The filler $\tilde{f}_{n, m}$ will be constructed in Theorem 15.17, In order to define it we consider the morphisms

$$
\Pi_{k+n, *}\left(X_{k} \mid \Omega^{n} X_{0}\right) \odot \Pi_{l+m, *}\left(Y_{l} \mid \Omega^{m} Y_{0}\right)
$$

defined on the (0)-level by

$$
\begin{gathered}
\mathbb{Z}_{\text {nil }}\left[\Omega^{n} X_{0}\right] \odot \mathbb{Z}_{\text {nil }}\left[\Omega^{m} Y_{0}\right] \\
\mathbb{Z}_{\text {nil }}\left[\left(\Omega^{n} X_{0}\right) \wedge\left(\Omega^{m} Y_{0}\right)\right] \\
\\
\mathbb{Z}_{\text {nil }}[\wedge] \\
\mathbb{Z}_{\text {nil }}\left[\Omega^{n+m}\left(X_{0} \wedge Y_{0}\right)\right] \\
\mathbb{Z}_{\text {nil }}\left[\Omega^{n+m} \jmath_{0,0}\right] \\
\mathbb{Z}_{\text {nil }}\left[\Omega^{n+m}(X \wedge Y)_{0}\right]
\end{gathered}
$$

Here the first arrow is the isomorphism in BM07, 3,7] and $\wedge:\left(\Omega^{n} X_{0}\right) \wedge\left(\Omega^{m} Y_{0}\right) \rightarrow$ $\Omega^{n+m}\left(X_{0} \wedge Y_{0}\right)$ is the map defined by $\left(f: S^{n} \rightarrow X_{0}\right) \wedge\left(g: S^{m} \rightarrow Y_{0}\right)=f \wedge$ $g: S^{n+m} \rightarrow X_{0} \wedge Y_{0}$. Moreover, the morphism (15.6) is induced on the 1-level by

$$
\begin{gathered}
\Pi_{k+n, *}\left(X_{k}\right) \odot \Pi_{l+m, *}\left(Y_{l}\right) \\
\qquad \begin{array}{l}
\mid \\
\Pi_{k+n+l+m, *}\left(X_{k} \wedge Y_{l}\right)
\end{array} \\
\Pi_{k+n+l+m, *}(X \wedge Y)_{k+l} \\
\prod_{k+l+n+m, *}(X \wedge Y)_{k+l}
\end{gathered}
$$

Here the last arrow is given by the right action of the sign group $\operatorname{Sym}_{\square}(k+l+n+m)$.

Lemma 15.9. The morphism (15.6) is well defined.

Proof. We have to check that the composite (15.7) is compatible with (15.8) so that (15.6) is indeed defined. This follows from the fact that given maps $f: S^{n} \rightarrow X_{0}$, 
$g: S^{m} \rightarrow Y_{0}$ the following diagram commutes

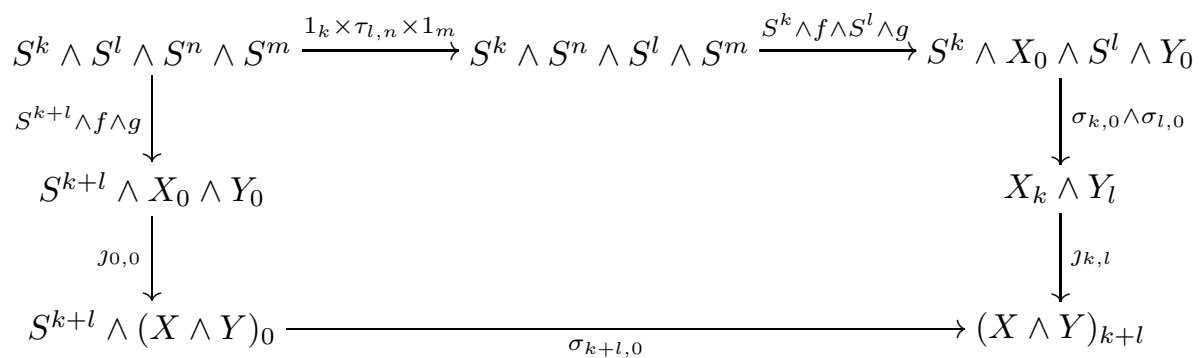

Theorem 15.10. Suppose that $E$ is a pointed space, $\varphi: E \rightarrow \Omega^{n+m} X$ is a pointed weak equivalence and the symmetric group $\operatorname{Sym}(n)$ acts from the left on $X$ in such a way that the adjoint

$$
\psi: S^{n} \wedge S^{m} \wedge E \longrightarrow X
$$

of $\varphi$ is equivariant with respect to the left action of $\operatorname{Sym}(n)$ on the sphere $S^{n}$. Suppose also that $m \geq 1$. Then for any $\tau \in \operatorname{Sym}(n)$ the two possible composites in the diagram

$$
\Pi_{n+m, *}(X \mid E) \stackrel{\varphi_{\#}}{\longrightarrow} \Pi_{n+m, *} X \underset{\left(\tau \times 1_{m}\right)^{*}}{\stackrel{\left(\Pi_{n+m, *} \tau\right.}{\longrightarrow}} \Pi_{n+m, *} X
$$

coincide.

Proof. The theorem is obvious in degrees 0 and ee. Let us check the degree 1 case. Obviously the statement of Theorem 15.10 is non-trivial just in case $n \geq 2$ so we can suppose that we are within this range, and hence $n+m \geq 3$.

Let $[f, F] \in \Pi_{n+m, 1}(X, E)$. This element is represented by a map

$$
f: S^{1} \longrightarrow \vee_{E} S^{1}
$$

such that the image of $\left(\pi_{1} f\right)(1) \in\langle E\rangle$ in the quotient $\langle E\rangle_{n i l}$ is $\partial[f, F]$, and a track

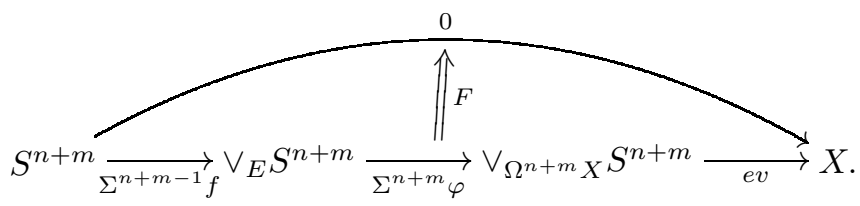

Here $\varphi$ is the underlying map of $\varphi$ above between pointed discrete sets.

On the one hand the element $\left(\Pi_{n+m, *} \tau\right) \varphi_{\#}[f, F]$ is represented by $(15.11)$

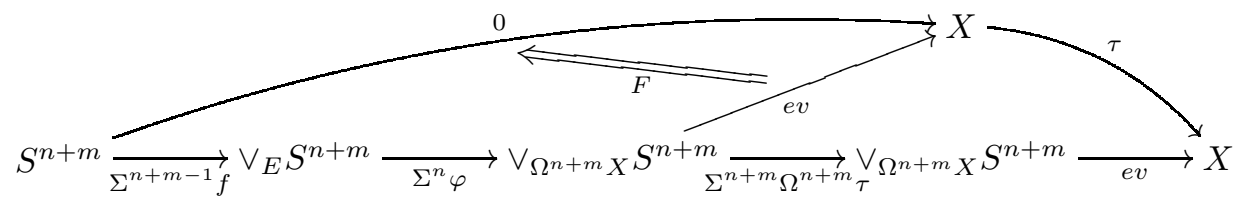

Here $\Omega^{n+m} \tau$ is also regarded as a map between pointed discrete sets. 


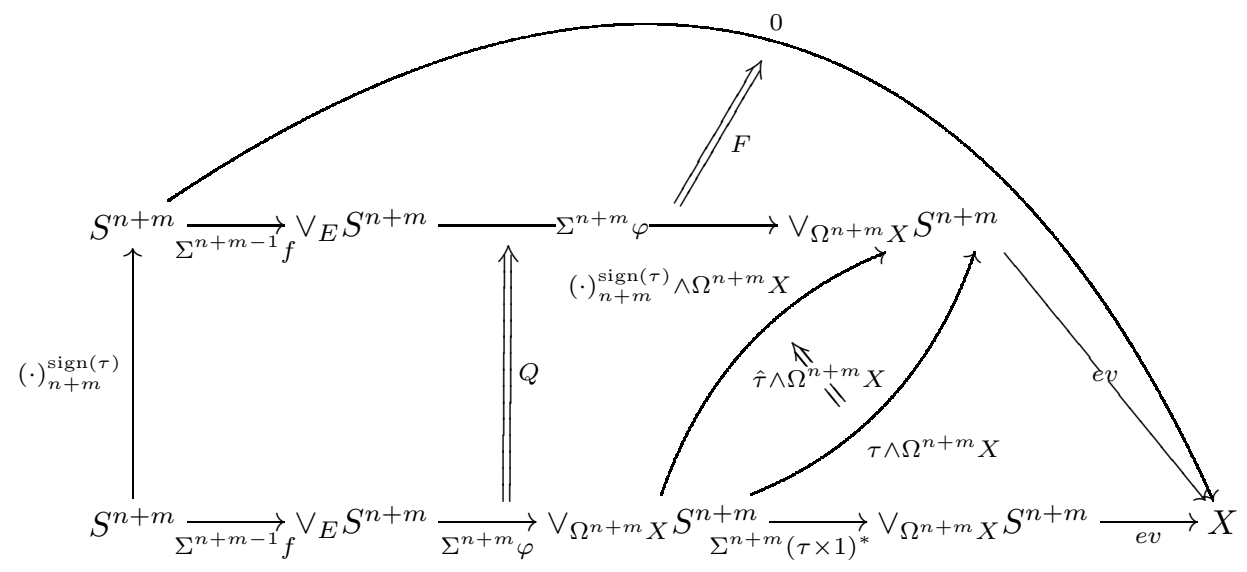

Here $\Omega^{n+m} X$ is regarded as a discrete pointed set, $(\tau \times 1)^{*}: \Omega^{n+m} X \rightarrow \Omega^{n+m} X$ is the pointed map induced by precomposition with $\tau \times 1_{m}=\tau \wedge S^{m}: S^{n+m} \rightarrow$ $S^{n+m},(\cdot)_{n+m}^{\operatorname{sign}(\tau)}: S^{n+m} \rightarrow S^{n+m}$ is the $(n+m-1)$-fold suspension of the map $(\cdot)^{\operatorname{sign}(\tau)}: S^{1} \rightarrow S^{1}$ where we use the multiplicative topological abelian group structure of $S^{1}, \hat{\tau}: \tau \times 1_{m} \Rightarrow(\cdot)_{n+m}^{\operatorname{sign}(\tau)}$ is any track, and $Q$ is the unique track with Hopf invariant

$$
\operatorname{Hopf}(Q)=-\left(\hat{\otimes}^{2} \mathbb{Z}[\varphi]\right)\left(\begin{array}{c}
\operatorname{sign}(\tau) \\
2
\end{array}\right) H \partial[f, F]
$$

Since $\psi$ is $\operatorname{Sym}(n)$-equivariant we have the equation

$$
\left(\Omega^{n} \tau\right) \varphi=\left(\tau \times 1_{m}\right)^{*} \varphi .
$$

We use this equation in order to simplify diagram (15.12) by using Lemma 15.16 below.

The hypothesis $m \geq 1$ is also needed in order to apply Lemma 15.16 below. By Lemma 15.16 the track (15.12) coincides with

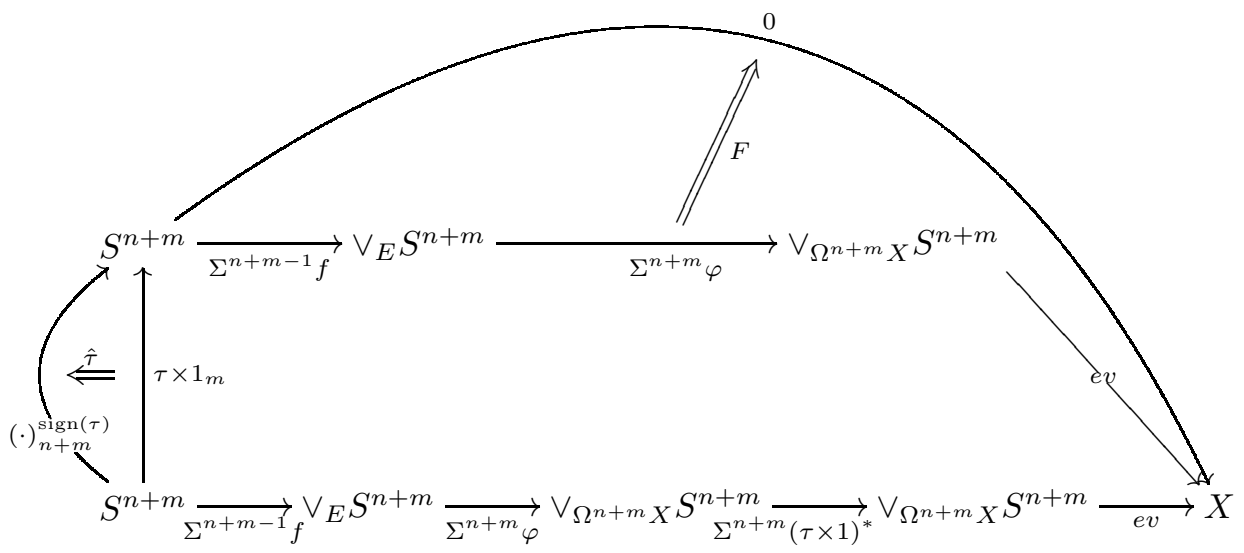

Any track horizontally composed with a trivial map becomes a trivial track, therefore in order to complete the proof it is enough to check that

$$
\tau F=F(\tau \times 1) .
$$


The track $F$ can be regarded as the relative homotopy class of a path in $\Omega^{n+m} X$ between two points comming from $E$. Since $\varphi$ is a weak equivalence the whole path comes from $E$ up to homotopy. Moreover, by (15.13) the two possible composites in the diagram

$$
E \stackrel{\varphi}{\longrightarrow} \Omega^{n+m} X \underset{\Omega^{n+m} \tau}{\stackrel{\left(\tau \times 1_{m}\right)^{*}}{\longrightarrow}} \Omega^{n+m} X
$$

coincide. Hence (d) follows.

The following lemma is used in the proof of Theorem 15.10 above. The map $(\cdot)_{n+m}^{\operatorname{sign}(\tau)}$ and the track $\hat{\tau}$ in the statement of the lemma can also be found in the proof of Theorem 15.10 .

Lemma 15.16. Let $m \geq 1$. Given two pointed sets $A, B$ and a map $f: \vee_{A} S^{1} \rightarrow$ $\vee_{B} S^{1}$ the Hopf invariant of the composite track $M(f)$

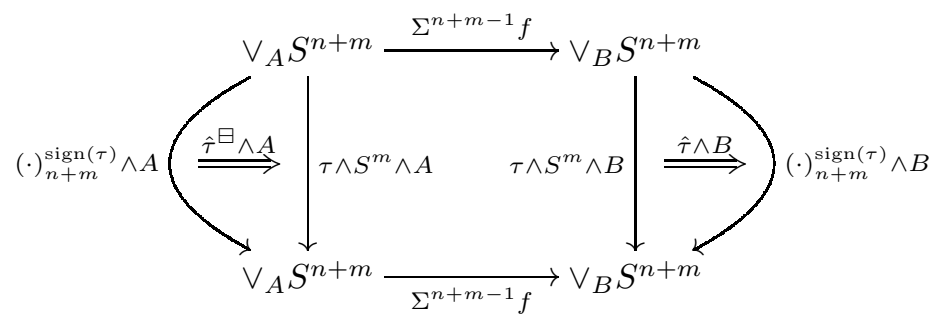

is defined by the equation, $a \in A$,

$$
\operatorname{Hopf}(M(f))(a)=\left(\begin{array}{c}
\operatorname{sign}(\tau) \\
2
\end{array}\right) H\left(\left(\pi_{1} f\right)_{n i l}(a)\right) \in \otimes^{2} \mathbb{Z}[B],
$$

where $\left(\pi_{1} f\right)_{n i l}(a)$ denotes the image under $\pi_{1} f$ of the generator $a \in\langle A\rangle \cong \pi_{1}\left(\vee_{A} S^{1}\right)$ projected to $\langle B\rangle_{\text {nil }}$.

Proof. By using similar techniques to [BM07, 16] one can check that it is enough to prove the statement for $f=(\cdot)^{-1}: S^{1} \rightarrow S^{1}$ the complex inversion. We have that

$$
\left(\begin{array}{c}
\operatorname{sign}(\tau) \\
2
\end{array}\right) H(-1)=\left(\begin{array}{c}
\operatorname{sign}(\tau) \\
2
\end{array}\right) \in \mathbb{Z} / 2
$$

This coincides with $\operatorname{Hopf}\left(M\left((\cdot)^{-1}\right)\right)$ provided the following identity holds in the positive pin group $\operatorname{Pin}_{+}(n+m)$ as defined in BM06c, 6.6]

$$
e_{n+m} \hat{\tau}=(-1)^{\left(\begin{array}{c}
\operatorname{sign}(\tau) \\
2
\end{array}\right)} \hat{\tau} e_{n+m} \text {. }
$$

The positive pin group is defined in BM06c, 6.6] as a subgroup of units in the Clifford algebra $C_{+}(n+m)$ with generators $e_{1}, \ldots, e_{n+m}$ associated to the Euclidean scalar product in $\mathbb{R}^{n+m}$. See also BM06c, 6.11] to see how $\hat{\tau}$ defines an element in $\operatorname{Pin}_{+}(n+m)$. Now the equation can be checked by using the defining relations of $C_{+}(n+m)$, see [BM06c, 6.6], and the fact that $\hat{\tau}$ does not depend on $e_{n+m}$ as an element in $C_{+}(n+m)$.

We use Theorem 15.10 to prove the following result.

Theorem 15.17. For any morphism of symmetric spectra $f: X \wedge Y \rightarrow U$ with $U$ fibrant the composite of (15.6) with the morphism

$$
\begin{gathered}
\Pi_{k+l+n+m, *}\left((X \wedge Y)_{k+l} \mid \Omega^{n+m}(X \wedge Y)_{0}\right) \\
\prod_{k+l+n+m, *}\left(U_{k+l} \mid \Omega^{n+m} U_{0}\right)
\end{gathered}
$$


induced by $f$ defines a morphism in $\mathbf{q p m}$

$$
\tilde{f}_{n, m}: \pi_{n, *} X \odot \pi_{m, *} Y \longrightarrow \pi_{n+m, *} U
$$

by taking colimits over $k, l \geq 0$.

Proof. The map $f$ clearly induces a morphism (a) which is $\mathbb{Z}_{n i l}\left[\Omega^{n+m} f_{0}\right]$ on the (0)-level and which is induced by $\Pi_{k+l+n+m, *} f$ on the 1-level.

In order to check that the morphism (b) is defined in the colimit it is enough to check that

$$
f_{*} \sigma_{*}\left(1_{S^{1}} \Lambda\left(1_{k} \times \tau_{l, m} \times 1_{n}\right)^{*}\left(\jmath_{k, l}\right)_{*}(a \unrhd b)\right)
$$

is equal to both

$$
\begin{gathered}
\left.f_{*}\left(1_{1+k} \times \tau_{l, m} \times 1_{n}\right)^{*}\left(\jmath_{1+k, l}\right)_{*}\left(\sigma_{*}\left(1_{S^{1}} \triangle a\right) \underline{\wedge}\right)\right), \\
f_{*}\left(1_{k} \times \tau_{1+l, m} \times 1_{n}\right)^{*}\left(\jmath_{k, 1+l}\right)_{*}\left(a \triangle \sigma_{*}\left(1_{S^{1}} \underline{\wedge} b\right)\right),
\end{gathered}
$$

at least for $k, l$ sufficiently large. We will see that equality $(\mathrm{c})=(\mathrm{d})$ holds for any $k$ and $l$, while we have to require $l \geq 1$ to check that $(\mathrm{c})=(\mathrm{e})$.

In order to check $(\mathrm{c})=(\mathrm{d})$ we consider the following equations

$$
\begin{aligned}
\text { (c) } & =f_{*}\left(1_{1+k} \times \tau_{l, m} \times 1_{n}\right)^{*} \sigma_{*}\left(1_{S^{1}} \triangle\left(\jmath_{k, l}\right)_{*}(a \triangle b)\right) \\
& =f_{*}\left(1_{1+k} \times \tau_{l, m} \times 1_{n}\right)^{*} \sigma_{*}\left(S^{1} \wedge \jmath_{k, l}\right)_{*}\left(1_{S^{1}} \underline{\wedge}(a \triangle b)\right) \\
& =\text { (d). }
\end{aligned}
$$

For the first equality we use the fact that the sign group action on secondary homotopy groups of spaces is natural and that the smash product operation is equivariant, see BM07 7.1 and 8.13. For the second one we use again the naturality of the smash product operation. For the last one we use the fact that the following diagram commutes

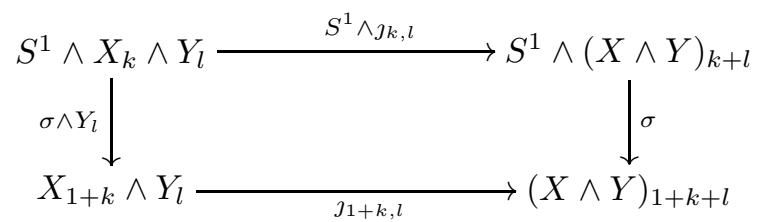

Let us now check $(\mathrm{c})=(\mathrm{e})$ under the assumption $l \geq 1$. Here we need to assume that $U$ is fibrant in order to apply Theorem 15.10, Let $\tau_{\wedge}: X_{k} \wedge S^{1} \cong$ $S^{1} \wedge X_{k}$ be the symmetry isomorphism for the smash product of pointed spaces. The commutativity rule for the smash product operation on secondary homotopy groups, see BM07] 7.3., and the fact that $H\left(1_{S^{1}}\right)=0$ show that

$$
\begin{aligned}
\left(\tau_{\wedge}\right)_{*}\left(a \triangle 1_{S^{1}}\right) & =\tau_{1, k}^{*}\left(1_{S^{1}} \wedge a\right) \\
& =\tau_{1, k}^{*}\left(1_{S^{1}} \triangle a\right) .
\end{aligned}
$$

Using this equality together with the commutativity of

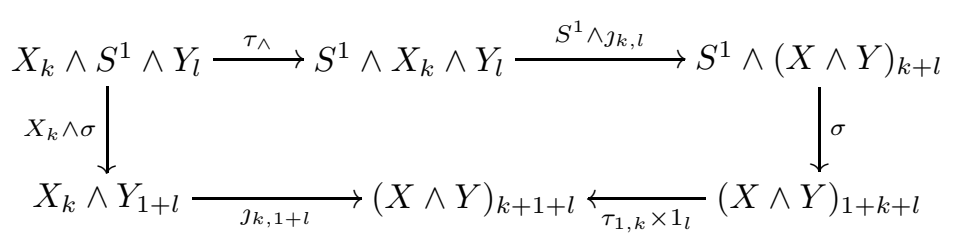


and the arguments used to check $(\mathrm{c})=(\mathrm{d})$ one can obtain the first equality in the following chain of equations.

$$
\begin{aligned}
(\mathrm{e})= & f_{*}\left(1_{k} \times \tau_{1+l, n} \times 1_{m}\right)^{*} \\
& \left(\tau_{k+n, 1} \times 1_{l+m}\right)^{*}\left(\tau_{1, k} \times 1_{l}\right)_{*} \sigma_{*}\left(1_{S^{1}} \triangle\left(\jmath_{k, l}\right)_{*}(a \triangle b)\right) \\
= & \left(1_{k} \times \tau_{1+l, n} \times 1_{m}\right)^{*} \\
& \left(\tau_{k+n, 1} \times 1_{l+m}\right)^{*}\left(\tau_{1, k} \times 1_{l}\right)_{*} \\
& \left(1_{1+k} \times \tau_{n, l} \times 1_{m}\right)^{*} \\
& f_{*} \sigma_{*}\left(1_{S^{1}} \wedge\left(1_{k} \times \tau_{l, n} \times 1_{m}\right)^{*}\left(\jmath_{k, l}\right)_{*}(a \triangle b)\right) \\
= & \left(1_{k} \times \tau_{1+l, n} \times 1_{m}\right)^{*} \\
& \left(\tau_{k+n, 1} \times 1_{l+m}\right)^{*}\left(\tau_{1, k} \times 1_{l}\right)_{*}\left(1_{1+k} \times \tau_{n, l} \times 1_{m}\right)^{*} \\
& \left(\tau_{k, 1} \times 1_{l}\right)_{*}\left(\tau_{1, k} \times 1_{l}\right)_{*} \\
& f_{*} \sigma_{*}\left(1_{S^{1}} \triangle\left(1_{k} \times \tau_{l, n} \times 1_{m}\right)^{*}\left(\jmath_{k, l}\right)_{*}(a \triangle b)\right) \\
\stackrel{(\mathrm{f})}{=} & \left(1_{k} \times \tau_{1+l, n} \times 1_{m}\right)^{*} \\
& \left(\tau_{k+n, 1} \times 1_{l+m}\right)^{*}\left(\tau_{1, k} \times 1_{l}\right)_{*} \\
& \left(1_{1+k} \times \tau_{n, l} \times 1_{m}\right)^{*} \\
& \left(\tau_{k, 1} \times 1_{l}\right)_{*}\left(\tau_{1, k} \times 1_{l+n+m}\right)^{*} \\
& f_{*} \sigma_{*}\left(1_{S^{1}} \triangle\left(1_{k} \times \tau_{l, n} \times 1_{m}\right)^{*}\left(\jmath_{k, l}\right)_{*}(a \triangle b)\right) \\
= & (\mathrm{c})
\end{aligned}
$$

In the second and the third steps we introduce permutations which cancel each other. For the last equality we use the identities

$$
\begin{aligned}
\left(\tau_{1, k} \times 1_{l+n+m}\right)\left(1_{1+k} \times \tau_{n, l} \times 1_{m}\right)\left(\tau_{k+n, 1} \times 1_{l+m}\right)\left(1_{k} \times \tau_{1+l, n} \times 1_{m}\right) & =1, \\
\left(\tau_{1, k} \times 1_{l}\right)\left(\tau_{k, 1} \times 1_{l}\right) & =1 .
\end{aligned}
$$

For (f) we use the hypothesis $l \geq 1$ and Theorem 15.10 applied to the map

$$
\varphi: \Omega^{n+m} U_{0} \rightarrow \Omega^{1+k+l+n+m} U_{1+k+l}
$$

obtained by taking $\Omega^{n+m}$ on the adjoint of $\sigma^{1+k+l}: S^{1+k+l} \wedge U_{0} \rightarrow U_{1+k+l}$. The $\operatorname{map} \varphi$ is a weak equivalence since $U$ is fibrant. Moreover, the adjoint of $\varphi$ is the composite

$$
\psi: S^{1+k} \wedge S^{l} \wedge S^{n+m} \wedge \Omega^{n+m} U_{0} \longrightarrow S^{1+k} \wedge S^{l} \wedge U_{0} \stackrel{\sigma_{1+k+l}}{\longrightarrow} U_{1+k+l},
$$

where the first arrow is obtained by taking $S^{1+k} \wedge S^{l} \wedge-$ on the adjoint $S^{n+m} \wedge$ $\Omega^{n+m} U_{0} \rightarrow U_{0}$ of the identity map on $\Omega^{n+m} U_{0}$, therefore $\psi$ is $\operatorname{Sym}(1+k)$-equivariant with respect to, the action of $\operatorname{Sym}(1+k)$ on $S^{1+k}$ by permutation of corrdinates, and the pull-back action of $\operatorname{Sym}(1+k+l)$ on $U_{1+k+l}$ along the inclusion $-\times 1_{l}: \operatorname{Sym}(1+k) \rightarrow \operatorname{Sym}(1+k+l)$.

One now readily checks that the homomorphism $\tilde{f}_{n, m}$ given by Theorem 15.17 proves Theorem 15.5 .

\section{Proof of Theorems 6.4, 7.4, and 9.12}

Since the notation $\pi_{*, *}$ is also used in the statement of Theorems 6.4, 7.4, and 9.12 we denote in this section the secondary homotopy groups of spectra defined in the previous section by $\bar{\pi}_{*, *}$.

Proof of Theorem 6.4. Let $L$ be a fibrant replacement functor in the model category of ring spectra. We define the functor $\pi_{*, *}$ in the statement of Theorem 6.4 as the composite $\bar{\pi}_{*, *} L$. Given a ring spectrum $R$ the ring multiplication $\mu: L R \wedge L R \rightarrow$ 
$L R$ and Theorem 15.5 induce a quadratic pair algebra structure on $\bar{\pi}_{*, *} L R$ with multiplication

$$
\mu_{*, *}: \bar{\pi}_{*, *} L R \odot \bar{\pi}_{*, *} L R \longrightarrow \bar{\pi}_{*, *} L R .
$$

Moreover, $\bar{\pi}_{0, *} S^{0} \cong \overline{\mathbb{Z}}_{n i l}$, and the unit $\nu: S^{0} \rightarrow L R$ of the ring spectrum $L R$ induces the unit of the quadratic pair algebra $\bar{\pi}_{*, *} L R$, which is the image of $1 \in \mathbb{Z}=\left(\overline{\mathbb{Z}}_{n i l}\right)_{0}$ by the morphism

$$
\overline{\mathbb{Z}}_{n i l} \cong \bar{\pi}_{0, *} S^{0} \hookrightarrow \bar{\pi}_{*, *} S^{0} \stackrel{\bar{\pi}_{*, *} \nu}{\longrightarrow} \bar{\pi}_{*, *} L R .
$$

The axioms of a quadratic pair algebra are satisfied since $\bar{\pi}_{*, *} L R$ is a monoid in the symmetric monoidal category $\mathbf{q p m}_{H}^{\mathbb{N}}$ of $\mathbb{N}$-graded 0-good quadratic pair algebras. This follows from Theorem 6.4 and the fact that secondary homotopy groups of pointed spaces form a lax monoidal functor to $\mathbf{q p m}_{H}^{\mathbb{N}}$, as we show in [BM07.

Let us check the statement about Toda brackets and Massey products. For the definition of Toda brackets in (1.2) we assumed $R$ to be fibrant. In this proof we do not make this assumption, therefore we replace $R$ in (1.2) by the fibrant replacement $L R$.

The right $L R$-module maps $\bar{a}, \bar{b}, \bar{c}$ in (1.2) are the same as maps of pointed spaces $\bar{a}: S^{p} \rightarrow L R_{0}, \bar{b}: S^{q} \rightarrow L R_{0}, \bar{c}: S^{r} \rightarrow L R_{0}$. These pointed maps are basis elements in the level 0 of $\pi_{*, *} R$ representing $a, b, c$, as in Definition 6.3. Moreover, the tracks $e$ and $f$ in (1.2) can be regarded as tracks between pointed maps $e: \mu_{0}(\bar{a} \wedge \bar{b}) \Rightarrow 0$, $f: \mu_{0}(\bar{b} \wedge \bar{c}) \Rightarrow 0$. These tracks in Top* represent elements in $\Pi_{p+q, 1} L R_{0}$ and $\Pi_{q+r, 1} L R_{0}$, respectively, which project to level 1 elements in the colimit $\pi_{*, *} R$ also denoted by $e$ and $f$. These elements satisfy $\partial(e)=\mu_{0}(\bar{a} \wedge \bar{b})=\bar{a} \cdot \bar{b}$ and $\partial(f)=\mu_{0}(\bar{b} \wedge \bar{c})=\bar{b} \cdot \bar{c}$ as required in Definition 6.3. Finally we notice that the element $-e \cdot \bar{c}+\bar{a} \cdot f$ defining the Massey product corresponds exactly to the pasting of diagram (1.2), which defines the Toda bracket, hence they coincide.

The statement about the $k$-invariant follows from Proposition 15.4.

Proof of Theorem 7.4. Let $L$ be a fibrant replacement functor in the model category of ring spectra and let $L^{\prime}$ be a fibrant replacement in the model category of right $L R$-modules. We define the functor $\pi_{*, *}$ in the statement of Theorem 7.4 as the composite $\bar{\pi}_{*, *} L^{\prime}\left(-\wedge_{R} L R\right)$. See [MMSS01, 22.2] for the definition of $\wedge_{R}$. Given a right $R$-module $M$ the right action of the ring spectrum $L R$ on the module spectrum $L^{\prime}\left(M \wedge_{R} L R\right)$,

$$
\gamma: L^{\prime}\left(M \wedge_{R} L R\right) \wedge L R \rightarrow L^{\prime}\left(M \wedge_{R} L R\right),
$$

and Theorem 15.5 induce a right $\bar{\pi}_{*, *} L R$-module structure on $\bar{\pi}_{*, *} L^{\prime}\left(M \wedge_{R} L R\right)$ with multiplication

$$
\gamma_{*, *}: \bar{\pi}_{*, *} L^{\prime}\left(M \wedge_{R} L R\right) \odot \bar{\pi}_{*, *} L R \longrightarrow \bar{\pi}_{*, *} L R,
$$

compare the proof of Theorem 6.4 above. The axioms of a right $\bar{\pi}_{*, *} L R$-module are satisfied since $\bar{\pi}_{*, *} L^{\prime}\left(M \wedge_{R} L R\right)$ is right module over the monoid monoid $\bar{\pi}_{*, *} L R$ in the symmetric monoidal category $\mathbf{q p m}_{H}^{\mathbb{N}}$ of $\mathbb{N}$-graded 0 -good quadratic pair algebras. This follows from Theorem 6.4 and the fact that secondary homotopy groups of pointed spaces form a lax monoidal functor to $\mathbf{q p m} \mathbf{m}_{H}^{\mathbb{N}}$, as we show in BM07.

The statements about Massey products and Toda brackets follow as in the proof of Theorem 6.4. The identification of the product morphism (7.2) is an easy exercise.

Remark 16.1. Once defined the functor $\pi_{*, *}$ in Theorem 7.4 we explicitly indicate how to construct the morphism (11.13) in the proof of Theorem 11.7 from a trivial 
cofibration $f: R^{\left(n_{1}, \ldots, n_{k}\right)}=\left(S^{n_{1}} \vee \cdots \vee S^{n_{k}}\right) \wedge R \stackrel{\sim}{\longmapsto} X$.

$$
\begin{aligned}
& \left(\pi_{*, *} R\right)^{\left(n_{1}, \ldots, n_{k}\right)} \\
& \left(\left(\overline{\mathbb{Z}}_{n i l}, n_{1}\right) \vee \cdots \vee\left(\overline{\mathbb{Z}}_{n i l}, n_{k}\right)\right) \odot \bar{\pi}_{*, *} L R \\
& \downarrow \text { using } \imath_{n_{j}, 0} \text { in Remark } 15.2 \\
& \left(\bar{\pi}_{n_{1}, *} S^{n_{1}} \vee \cdots \vee \bar{\pi}_{n_{k}, *} S^{n_{k}}\right) \odot \bar{\pi}_{*, *} L R \\
& \text { f } \\
& \left(\bar{\pi}_{*, *} S^{n_{1}} \vee \cdots \vee \bar{\pi}_{*, *} S^{n_{k}}\right) \odot \bar{\pi}_{*, *} L R \\
& \downarrow \\
& \bar{\pi}_{*, *}\left(S^{n_{1}} \vee \cdots \vee S^{n_{k}}\right) \odot \bar{\pi}_{*, *} L R \\
& \text { Linduced by }\left(S^{n_{1}} \vee \cdots \vee S^{n_{k}}\right) \wedge L R \sim \sim_{\sim}^{\prime} L^{\prime}\left(\left(S^{n_{1}} \vee \cdots \vee S^{n_{k}}\right) \wedge L R\right) \text { and Thm. } 15.17 \\
& \bar{\pi}_{*, *} L^{\prime}\left(\left(S^{n_{1}} \vee \cdots \vee S^{n_{k}}\right) \wedge L R\right) \\
& \pi_{*, *}\left(\left(S^{n_{1}} \vee \cdots \vee S^{n_{k}}\right) \wedge R\right) \\
& \downarrow \pi_{*, *} f \\
& \pi_{*, *} X
\end{aligned}
$$

In the statement of the following lemma we consider the interval 1-cell complex $K^{1}$, and the 2-cell complexes with the shape of a triangle $K^{2}$, a hexagon $K^{3}$, and a disc $K^{4}$.
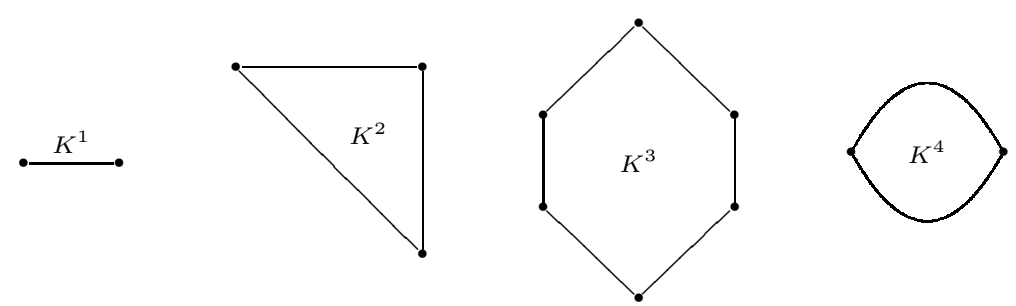

Moreover, given space $K$ we write $K_{+}$for the disjoint union of $K$ with an outer base-point. The smash product of a pointed space $K$ and a symmetric spectrum $X$ is the symmetric spectrum defined by $(K \wedge X)_{n}=K \wedge X_{n}$. We denote by $\tau_{\wedge}$ to the symmetry isomorphism for the smash product of spectra.

Lemma 16.2. Let $L$ be a fibrant replacement functor in the category of ring spectra. Given a commutative ring spectrum $Q$ the multiplication $\mu: L Q \wedge L Q \rightarrow L Q$ in $L Q$ needs not be commutative, but there is a commuting homotopy $\alpha_{1}: K_{+}^{1} \wedge L Q \wedge L Q \rightarrow$ $L Q$ which is coherent in the following sense: there are maps $\alpha_{2}: K_{+}^{2} \wedge L Q \wedge L Q \rightarrow$ $L Q$ and $\alpha_{3}: K_{+}^{3} \wedge L Q \wedge L Q \wedge L Q \rightarrow L Q$ defined over the boundary of $K^{1}$ and $K^{2}$ as indicated in the diagram below. Moreover, the commuting homotopy is natural in the following sense: given a map of commutative ring spectra $f: Q \rightarrow Q^{\prime}$ if $\alpha_{1}$ and $\alpha_{1}^{\prime}$ denote respective commuting homotopies then there is a map $\alpha_{4}: K_{+}^{4} \wedge L Q \wedge$ $L Q \rightarrow L Q^{\prime}$ defined over the boundary of $K^{4}$ as indicated in the diagram below. Furthermore, the map $\alpha_{i}$ can be chosen so that the restriction to $Q$ along the trivial cofibration $Q \stackrel{\sim}{\longmapsto} L Q$ is constant over $K_{i}, i=1,2,3,4$.
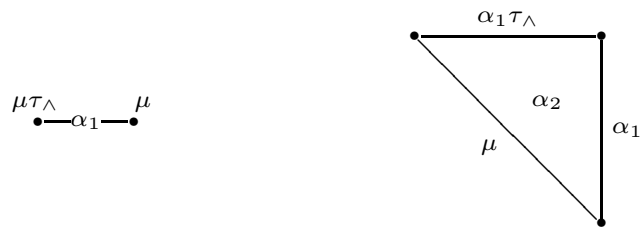

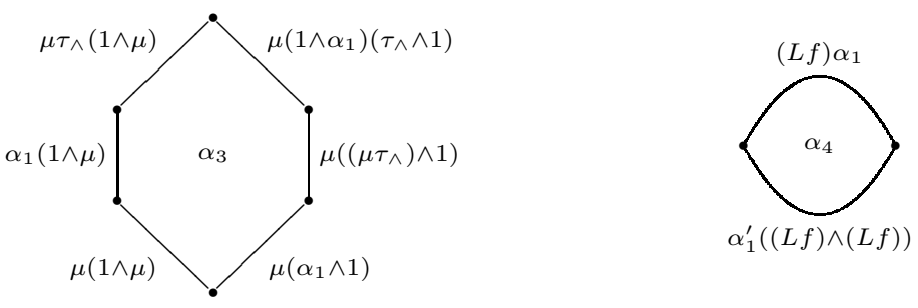

Here the edges labelled with a map which only depends on $\mu$ and $\tau_{\wedge}$ are constant homotopies on the indicated map.

Proof. Let $P$ be the push-out

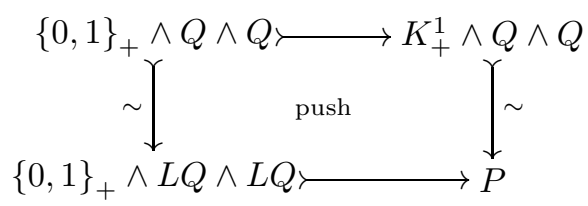

The inclusion of the boundary $\{0,1\} \longmapsto K^{1}$ and $Q \stackrel{\sim}{\hookrightarrow} L Q$ induce a trivial cofibration

$$
j: P \stackrel{\sim}{\longmapsto} K_{+}^{1} \wedge L Q \wedge L Q .
$$

Consider the composite map

$$
a: K_{+}^{1} \wedge Q \wedge Q \longrightarrow Q \wedge Q \stackrel{\text { mult. }}{\longrightarrow} \stackrel{\sim}{\sim} L Q
$$

where the first arrow is given by the map collapsing the interval $K^{1}$ to a point. Consider also the map

$$
b:\{0,1\}_{+} \wedge L Q \wedge L Q \longrightarrow L Q
$$

which restricted to $\{1\}$ is $\mu$ and restricted to $\{0\}$ is $\mu \tau_{\wedge}$. The maps $a$ and $b$ induce a map

$$
a \cup b: P \longrightarrow L Q
$$

The map $\alpha_{1}$ is defined as a lift of the following diagram

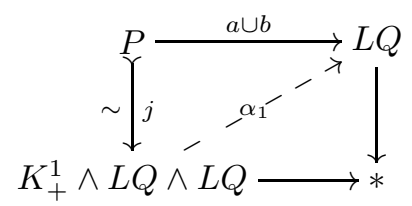

Notice that the procedure for the construction of $\alpha_{1}$ consists of defining it over the restriction to $Q$ and on the boundary of $K^{1}$ according to the statement. Then $\alpha_{1}$ is given by the usual lifting diagram in a model category. The same procedure can be used for the definition of $\alpha_{2}, \alpha_{3}$, and $\alpha_{4}$.

Proof of Theorem 9.12. Let $L$ be a fibrant replacement functor in the category of ring spectra. Given a commutative ring spectrum $Q$ we have to promote the quadratic pair algebra $\bar{\pi}_{*, *} L Q$ in the proof of Theorem 6.4 to an $E_{\infty}$-quadratic pair algebra. As we showed in the previous section the quadratic pair module $\bar{\pi}_{n, *} L Q$ carries an action of the symmetric track group $\operatorname{Sym}_{\square}(n)$. The multiplication, defined by Theorem 15.5. is equivariant with respect to the sign group homomorphism (8.5), i.e. $\bar{\pi}_{*, *} L Q$ is a monoid in the category $\mathbf{q p m}_{H}^{\mathrm{Sym}} \square$ of enhanced symmetric sequences of 0 -good quadratic pair modules. Now consider the map $\alpha_{1}$ in the statement of Lemma 16.2 By Theorem 15.17, and using that the sphere spectrum $S$ is the unit of the smash product, the map $\alpha_{1}$ yields a morphism

$$
\bar{\pi}_{0, *}\left(K_{+}^{1} \wedge S\right) \odot \bar{\pi}_{*, *} L Q \odot \bar{\pi}_{*, *} L Q \longrightarrow \bar{\pi}_{*, *} L Q .
$$


The "interval" quadratic pair module $\mathbb{I}$ in [BM07, 5] is 0-free. The basis at level 0 is the set of vertices of $K_{1}$, i.e. $\{0,1\}$. On level 1 it is generated by the edge $e$ of $K^{1}$ and $\partial(e)=-(0)+(1)$. This correspondence between the generators of $\mathbb{I}$ and $K^{1}$ yields a morphism $v: \mathbb{I} \rightarrow \Pi_{0, *} K_{+}^{1}$, see [BM07, 19]. Conposing $v$ with the projection to the colimit $\phi_{0}: \Pi_{0, *} K_{+}^{1} \rightarrow \bar{\pi}_{0, *}\left(K_{+}^{1} \wedge S\right)$ we obtain a new morphism

$$
\mathbb{I} \odot \bar{\pi}_{*, *} L Q \odot \bar{\pi}_{*, *} L Q \longrightarrow \bar{\pi}_{*, *} L Q .
$$

Such a morphism is the same as a track to the multiplication of $\bar{\pi}_{*, *} L Q$ from the opposite multiplication. The maps $\alpha_{2}$ and $\alpha_{3}$ in Lemma 16.2 show that this track satisfies the idempotence and the hexagon conditions, therefore $\bar{\pi}_{*, *} L Q$ is an $E_{\infty^{-}}$ quadratic pair algebra, see Remark 9.13. The map $\alpha_{4}$ in Lemma 16.2 shows the naturaluty of $\smile_{1}$ with respect to morphisms between commutative ring spectra.

For the statement about the smash product we notice that $\bar{a}$ in diagram (1.3) is the same as a pointed map $\bar{a}: S^{2 n} \rightarrow L Q_{0}$ which, regarded as a basis element in $\bar{\pi}_{2 n, 0} L Q$, represents $a \in \pi_{2 n} Q=$ Coker $\partial$. Since $\bar{a}$ is in the basis $H(\bar{a})=0$. This simplifies the definition of the algebraic cup-one square

$$
S q_{1}(a)=-(\bar{a} \cdot \bar{a}) \cdot[\hat{\tau}]+\bar{a} \smile_{1} \bar{a},
$$

see Definition 9.10, Let us recall how these summands are explicitly defined.

An element $\hat{\tau} \in \operatorname{Sym}_{\square}(n)$ as in Definition 9.10 is the same as a track $\hat{\tau}$ as in diagram (1.3). Moreover, $\bar{a} \cdot \bar{a}=\mu_{0}(\bar{a} \wedge \bar{a})$. The element $(\bar{a} \cdot \bar{a}) \cdot[\hat{\tau}]$ is the projection to the colimit of $\left\langle\mu_{0}(\bar{a} \wedge \bar{a}), \hat{\tau}\right\rangle \in \Pi_{4 n, 1} L Q_{0}$ in the sense of [BM06c, 4.5].

The map $\alpha_{1}$ in Lemma 16.2 is given on degree 0 by a homotopy $\left(\alpha_{1}\right)_{0}$ to the product on $L Q_{0}$ from the opposite product. Such a homotopy induces a track of quadratic pair modules, which is a function $\left(\left(\alpha_{1}\right)_{0}\right)_{*}: \Pi_{4 n, 0} L Q_{0} \rightarrow \Pi_{4 n, 1} L Q_{0}$. The construction of $\left(\left(\alpha_{1}\right)_{0}\right)_{*}\left(\mu_{0}(\bar{a} \wedge \bar{a})\right)$ can be found in BM06a, 7.3]. The projection to the colimit of this element is $\bar{a} \smile_{1} \bar{a}$.

With this description of the summands in (a) one can check that (a) is the same as the pasting of diagram (1.3).

\section{Proof of Theorem 3.4}

In order to derive Theorem 3.4 from Theorem 9.12 we need some technical results.

In Remark 9.8 we indicated how the category of $E_{\infty}$-pair algebras can be regarded as a full subcategory of the category of $E_{\infty}$-quadratic pair algebras. This inclusion admits a reflection

$$
(-)^{a d c}:\left(E_{\infty} \text {-quadratic pair algebras }\right) \longrightarrow\left(E_{\infty} \text {-pair algebras }\right)
$$

defined as follows.

Let $C$ be an $E_{\infty}$-quadratic pair algebra. The quadratic pair module $C_{n, *}$ is a right $A\left(\operatorname{Sym}_{\square}(n)\right)$-module for all $n \geq 0$, therefore the additivization $C_{n, *}^{a d d}$ in the sense of (5.6) is a right $A\left(\operatorname{Sym}_{\square}(n)\right)^{a d d}$-module. The pair algebra $A\left(\operatorname{Sym}_{\square}(n)\right)^{a d d}$ is the inclusion of the two-sided ideal $I_{\text {sign }} \subset \mathbb{Z} \operatorname{Sym}(n)$ in the group-ring of the symmetric group which is the kernel of the $\operatorname{ring} \operatorname{homomorphism} \mathbb{Z} \operatorname{Sym}(n) \rightarrow \mathbb{Z}$ induced by the sign homomorphism sign: $\operatorname{Sym}(n) \rightarrow\{ \pm 1\}$. The pair module $C_{n, *}^{a d c}$ is defined by the following diagram with exact rows

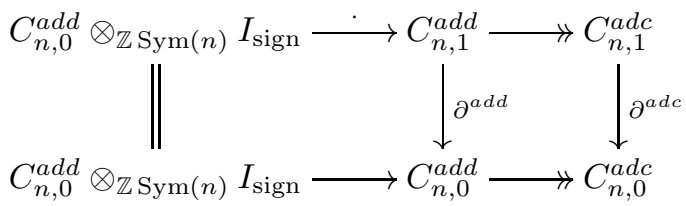

Here the arrow labeled with a dot $\cdot$ is defined by one of the multiplications given by the right $A\left(\operatorname{Sym}_{\square}(n)\right)^{a d d}$-module structure of $C_{n, *}^{a d d}$. The natural projections 
$C_{n, *} \rightarrow C_{n, *}^{a d d} \rightarrow C_{n, *}^{a d c}$ define an $E_{\infty}$-quadratic pair algebra morphism $C \rightarrow C^{a d c}$, the unit of the reflection. The product and the cup-one product in $C^{a d c}$ are determined by this fact.

Lemma 17.2. Let $C$ be an $E_{\infty}$-quadratic pair algebra with trivial $k$-invariants such that $C_{n, 0}^{a d d}$ is a flat right $\operatorname{Sym}(n)$-module for all $n \geq 0$. Then the natural projection $C \rightarrow C^{a d c}$ is a quasi-isomorphism.

Proof. Since $C_{n, *}$ has trivial $k$-invariant the natural projection $C_{n, *} \rightarrow C_{n, *}^{a d d}$ is a quasi-isomorphism by Proposition 5.7. Moreover, since $C_{n, 0}^{a d d}$ is flat as a right $\operatorname{Sym}(n)$-module then the lower horizontal arrow in the left of diagram (17.1) is injective. This implies that the upper one, labeled $\cdot$, is also injective, hence the "snake lemma" applied to diagram (17.1) implies that $C_{n, *}^{a d d} \rightarrow C_{n, *}^{a d c}$ is also a quasiisomorphism, and the lemma follows.

The technical condition on $C_{n, 0}^{a d d}$ in the statement of Lemma 17.2 is satisfied, up to a blow-up, by any $E_{\infty}$-quadratic pair algebra, as we show in the following lemma.

Lemma 17.3. Let $C$ be a 0 -free $E_{\infty}$-quadratic pair algebra. There is a 0 -free quadratic pair algebra $\widetilde{C}$ with $\widetilde{C}_{n, 0}^{\text {add }}$ a flat right $\operatorname{Sym}(n)$-module for all $n \geq 0$ and a natural projection $\widetilde{C} \rightarrow C$ which is a quasi-isomorphism.

Proof. Since $C$ is 0 -free there are pointed sets $E_{n}$ such that $C_{n,(0)}=\mathbb{Z}_{n i l}\left[E_{n}\right]$, $n \geq 0$. The laws of a module over a quadratic pair algebra show that for any $e \in E_{n}$ and $g \in \operatorname{Sym}(n)$ we have $H(e \cdot[g])=0$. The unique elements of $\left\langle E_{n}\right\rangle_{n i l}$ in the kernel of $H$ are the elements of the basis $E_{n}$, compare the proof of BJP05. Lemma 12]. Therefore the action of $\operatorname{Sym}(n)$ on $\left\langle E_{n}\right\rangle_{n i l}$ is induced by an action of $\operatorname{Sym}(n)$ on the pointed set $E_{n}$.

The right $\operatorname{Sym}(n)$-module $C_{n, 0}^{a d d}=\mathbb{Z}\left[E_{n}\right]$ needs not be flat. It would be flat if $E_{n}$ were a free pointed $\operatorname{Sym}(n)$-set. We are going to construct $\widetilde{C}$ by replacing $E_{n}$ by a free $\operatorname{Sym}(n)$-set without changing $h_{0}$ and $h_{1}$. Let

$$
\varphi_{n}: E_{n}^{\prime}=E_{n} \wedge\left(\operatorname{Sym}(n)_{+}\right) \longrightarrow E_{n}
$$

be the pointed map induced by the action of $\operatorname{Sym}(n)$ on $E_{n}$. This map is $\operatorname{Sym}(n)$ equivariant with respect to the action of $\operatorname{Sym}(n)$ on $E_{n}^{\prime}$ given by multiplication on the second coordinate of the smash product. Moreover, $E_{*}^{\prime}$ is a graded monoid with multiplication given coordinatewise by the product in $E_{*}$ and the cross product homomorphisms in (8.6). This multiplication is equivariant with respect to the cross product homomorphisms. Let $\varphi: E^{\prime} \rightarrow E$ be the graded map given by $\varphi_{n}$.

Since $E_{n}^{\prime}$ is a free $\operatorname{Sym}(n)$-set for all $n \geq 0$ the $E_{\infty}$-quadratic pair algebra $\widetilde{C}=$ $\varphi^{*} C$ in Lemma 14.5 and the natural projection $\varphi_{\#}: \widetilde{C} \rightarrow C$ satisfy the properties stated in the lemma.

Now we are ready to prove Theorem 3.4

Proof of Theorem 3.4. The $E_{\infty}$-quadratic pair algebra $\pi_{*, *} Q$ in Theorem 9.12 is 0 -free, therefore by Lemmas 17.2 and 17.3 the functorial $E_{\infty}$-pair algebra

$$
\pi_{*, *}^{a d c} Q=\left(\widetilde{\pi_{*, *} Q}\right)^{a d c}
$$

proves Theorem 3.4. The natural zig-zag of quasi-isomorphisms between the pair algebras $\pi_{*, *}^{a d c} Q$ and $\pi_{*, *}^{a d d} Q$ is obtained by applying the additivization functor (5.6) to the diagram

$$
\left.\widetilde{\left(\pi_{*, *} Q\right.}\right)^{a d c} \leftarrow \widetilde{\pi_{*, *} Q} \rightarrow \pi_{*, *} Q,
$$


i.e. it is

$$
\pi_{*, *}^{a d c} Q \nleftarrow\left(\widetilde{\pi_{*, *} Q}\right)^{a d d} \rightarrow \pi_{*, *}^{a d d} Q .
$$

For this we recall that we have defined $\pi_{*, *}^{a d d} Q$ as $\left(\pi_{*, *} Q\right)^{\text {add }}$ in the proof of Theorem 2.7 .

Remark 17.5. In order to deduce Theorem 4.12 from Theorem 10.6 one extends the results of this section to algebras over an $E_{\infty}$-quadratic pair algebra $C$. More precisely, the natural projection $C \rightarrow C^{a d c}$ induces an inclusion

$$
\left(C^{a d c} \text {-algebras }\right) \subset(C \text {-algebras }) .
$$

This inclusion admits a reflection

$$
(-)^{a d a}:(C \text {-algebras }) \longrightarrow\left(C^{a d c} \text {-algebras }\right)
$$

sending a $C$-algebra $B$ to the $C^{a d c}$-algebra $B^{a d a}$ obtained from the additivization $B^{a d d}$ by killing the action of the symmetric groups as in (17.1). Lemmas 17.2 and 17.3 admit a generalized version for $C$-algebras. By using this one can check as in the proof of Theorem 3.4 that the functor $\pi_{*, *}^{a d a}$ in Theorem 4.12 can be obtained taking first $\pi_{*, *}$ in Theorem 10.6 and then applying $(-)^{a d a}$ to a blow-up of the resulting $E_{\infty}$-quadratic pair algebra.

\section{REFERENCES}

[Ale72] J. C. Alexander, Cobordism Massey products, Trans. Amer. Math. Soc. 166 (1972), 197-214.

[Bas99] M. Basterra, André-Quillen cohomology of commutative S-algebras, J. Pure Appl. Algebra 144 (1999), no. 2, 111-143.

[Bau89] H.-J. Baues, Algebraic Homotopy, Cambridge University Press, 1989.

[Bau06] _ The algebra of secondary cohomology operations, Progress in Math. 247, Birkhäuser, 2006.

[BD89] H.-J. Baues and W. Dreckmann, The cohomology of homotopy categories and the general linear group, K-Theory 3 (1989), no. 4, 307-338.

[BE74] M. G. Barratt and P. J. Eccles, $\Gamma^{+}$-structures. I. A free group functor for stable homotopy theory, Topology 13 (1974), 25-45.

[BJ04] H.-J. Baues and M. Jibladze, Computation of the E E-term of the Adams spectral sequence, Preprint of the Max-Planck-Institut für Mathematik MPIM2004-53, http://arxiv.org/abs/math.AT/0407045, 2004.

[BJP05] H.-J. Baues, M. Jibladze, and T. Pirashvili, Quadratic algebra of square groups, Preprint of the Max-Planck-Institut für Mathematik MPIM2006-9, http://arxiv.org/abs/math.CT/0601777, 2005.

[BJP06] Third Mac Lane cohomology, Preprint of the Max-Planck-Institut für Mathematik MPIM2006-50, http://arxiv.org/abs/math.KT/0604447, 2006.

[BM02] H.-J. Baues and E. G. Minian, Crossed extensions of algebras and Hochschild cohomology, Homology Homotopy Appl. 4 (2002), no. 2, part 1, 63-82 (electronic), The Roos Festschrift volume, 1.

[BM05] H.-J. Baues and F. Muro, The homotopy category of pseudofunctors and translation cohomology, Preprint of the Max-Planck-Institut für Mathematik MPIM2005-39 http://arxiv.org/abs/math.KT/0505540, 2005.

[BM06a] Secondary homotopy groups, Preprint of the Max-Planck-Institut für Mathematik MPIM2006-36, http://arxiv.org/abs/math.AT/0604029, to appear in Forum Mathematicum, 2006.

[BM06b] - Some relations between secondary operations in ring spectra, in preparation, 2006.

[BM06c] - The symmetric action on secondary homotopy groups, Preprint of the Max-Planck-Institut für Mathematik MPIM2006-37, http://arxiv.org/abs/math.AT/0604030, 2006.

[BM07] - Smash products for secondary homotopy groups, Preprint of the Max-PlanckInstitut für Mathematik MPIM2006-38, http://arxiv.org/abs/math.AT/0604031, to appear in Applied Categorical Structures, 2007.

[BMR04] H.-J. Baues, E. G. Minian, and B. Richter, Crossed modules over operads and operadic cohomology, K-Theory 31 (2004), no. 1, 39-69. 
[Bor94] F. Borceux, Handbook of categorical algebra 2, Encyclopedia of Math. and its Applications, no. 51, Cambridge University Press, 1994.

[BP] H.-J. Baues and T. Pirashvili, Comparison of MacLane cohomology, Shukla and Hochschild, To appear in J. Reine Angew. Math.

[BP99] Quadratic endofunctors of the category of groups, Advances in Math. 141 (1999), 167-206.

[EM05] A. D. Elmendorf and M. A. Mandell, Rings, modules, and algebras in infinite loop space theory, Preprint, http://www.math.uiuc.edu/K-theory/0748/, 2005.

[GH99] T. Geisser and L. Hesselholt, Topological cyclic homology of schemes, Algebraic Ktheory (Seattle, WA, 1997), Proc. Sympos. Pure Math., vol. 67, Amer. Math. Soc., Providence, RI, 1999, pp. 41-87.

[Hel68] A. Heller, Stable homotopy categories, Bull. Amer. Math. Soc. 74 (1968), 28-63

[Hov99] M. Hovey, Model categories, Mathematical Surveys and Monographs, vol. 63, American Mathematical Society, Providence, RI, 1999.

[Hov01] _ Spectra and symmetric spectra in general model categories, J. Pure Appl. Algebra 165 (2001), no. 1, 63-127.

[JP86] M. A. Jibladze and T. I. Pirashvili, Some linear extensions of a category of finitely generated free modules, Soobshch. Akad. Nauk Gruzin. SSR 123 (1986), no. 3, 481484.

[Kri63] L. Kristensen, On secondary cohomology operations, Math. Scand. 12 (1963), 57-82.

[Laz01] A. Lazarev, Homotopy theory of $A_{\infty}$ ring spectra and applications to $M U$-modules, $K$-Theory 24 (2001), no. 3, 243-281.

[Lod98] J.-L. Loday, Cyclic homology, second ed., Grundlehren der Mathematischen Wissenschaften [Fundamental Principles of Mathematical Sciences], vol. 301, SpringerVerlag, Berlin, 1998, Appendix E by María O. Ronco, Chapter 13 by the author in collaboration with Teimuraz Pirashvili.

[May69] J. P. May, Matric Massey products, J. Algebra 12 (1969), 533-568.

[McC85] J. McCleary, A Users Guide to Spectral Sequences, Publish or Perish, Inc., Wilmington, DE, 1985.

[MMSS01] M. A. Mandell, J. P. May, S. Schwede, and B. Shipley, Model categories of diagram spectra, Proc. London Math. Soc. (3) 82 (2001), no. 2, 441-512.

[MT06] F. Muro and A. Tonks, The 1-type of a Waldhausen K-theory spectrum, Preprint of the Max-Planck-Institut für Mathematik MPIM2006-28, http://arxiv.org/abs/math.KT/0603544, 2006

[PW92] T. Pirashvili and F. Waldhausen, MacLane homology and topological Hochschild homology, J. Pure Appl. Algebra 82 (1992), no. 1, 81-98.

[Rav86] D. C. Ravenel, Complex cobordism and stable homotopy groups of spheres, Pure and Applied Mathematics, vol. 121, Academic Press Inc., Orlando, FL, 1986.

[Sag06] S. Sagave, Universal toda brackets of ring spectra, Ph.D. thesis, University of Bonn, 2006.

[Sch04] S. Schwede, Morita theory in abelian, derived and stable model categories, Structured ring spectra, London Math. Soc. Lecture Note Ser., vol. 315, Cambridge Univ. Press, Cambridge, 2004, pp. 33-86.

[Shi06] B. E. Shipley, HZ-algebra spectra are differential graded algebras, To appear in American Journal of Mathematics, http://www.math.uic.edu/ bshipley/zdga17.pdf 2006.

[Tod62] H. Toda, Composition methods in homotopy groups of spheres, Annals of Mathematics Studies, No. 49, Princeton University Press, Princeton, N.J., 1962.

[Tod71] Algebra of stable homotopy of $Z_{p}$-spaces and applications, J. Math. Kyoto Univ. 11 (1971), 197-251.

[Wal78] F. Waldhausen, Algebraic K-theory of topological spaces. I, Algebraic and geometric topology (Proc. Sympos. Pure Math., Stanford Univ., Stanford, Calif., 1976), Part 1, Proc. Sympos. Pure Math., XXXII, Amer. Math. Soc., Providence, R.I., 1978, pp. 3560.

[Whi49] J. H. C. Whitehead, Combinatorial homotopy II, Bull. Amer. Math. Soc. 55 (1949), 453-496.

Max-Planck-Institut für Mathematik, Vivatsgasse 7, 53111 Bonn, Germany

E-mail address: baues@mpim-bonn.mpg.de

Universitat de Barcelona, Departament d’Àlgebra i Geometria, Gran Via de les Corts Catalanes, 585, 08007 Barcelona, Spain

E-mail address: fmuro@ub.edu 